\title{
Introdução aos Métodos Algébricos Unidimensionais em Espectroscopia Molecular: Sistemas Diatômicos
}

\author{
Germán Ernesto Montoya Vargas
}

Dissertação apresentada ao Instituto de Física de São Carlos, da Universidade de São Paulo, para obtenção do título de Mestre em Ciências: Física Básica.

Orientador: Prof. Dr. Esmerindo de Sousa Bernardes 



\section{Agradecimentos}

- Agradeço a Deus por toda ajuda e opurtunidades.

- Gostaria de expressar meus sinceros agradecimentos ao meu orientador, Prof. Dr. Esmerindo de Sousa Bernardes pelo apoio incondicional, afinal sua ajuda foi de extrema valia para elaboração deste projeto.

- Agradeço à minha família, ainda que separados pela distância, reconfortaram-me com amor e me deram força nos momentos mais difíceis.

- Aos meus amigos da alma, Karen e Orlando, que fizeram minha estadia no Brasil muito calorosa. Algum dia espero poder retribuir a eles tudo o que fizeram por mim (eu sei como pagar-lhes).

- À todos meus amigos brasileiros, Lucas, Patrícia, Lia, Raphael, Petrus, Emmanuel, Alexandre, as duas Renatas, Juliana, e tantos outros que estão presentes em meu coração e que me acompanharam neste país proporcionando-me uma agradável estadia.

- À colônia colombiana com quem vivenciei momentos tão divertidos, em especial a William e Daniel.

- Y por último a este país lleno de verde y esperanza.

Este trabalho teve o apoio financeiro da Fundação de Amparo à Pesquisa do Estado de São Paulo (FAPESP) e do Conselho Nacional de Desenvolvimento Científico e Tecnológico (CNPq). 
Montoya Vargas, Germán Ernesto

Introdução aos Métodos Algébricos Unidimensionais em Espectroscopia Molecular: Sistemas Diatômicos-São Carlos, 2003.

79 p.

Dissertação (Mestrado)-Instituto de Física de São Carlos, 2003.

Orientador: Prof. Dr. Esmerindo de Sousa Bernardes

1. Métodos Algébricos 2. Álgebras de Lie 3. Espectroscopia Molecular 4. Moléculas Diatômicas

Métodos Matemáticos da Física. I. Título. 


\section{Conteúdo}

1 Introdução 1

2 Ferramentas Algébricas $\quad 5$

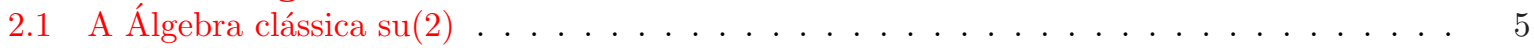

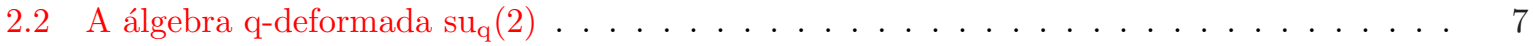

2.3 Estados coerentes para o grupo $\mathrm{SU}(2) \ldots \ldots \ldots \ldots \ldots \ldots \ldots$

2.4 Estados coerentes q-deformados . . . . . . . . . . . . . . . . . . . . . . . . . 10

2.5 Valores médios . . . . . . . . . . . . . . . . . . . . . . . . . 10

3 Modelo Algébrico Unidimensional 13

3.1 Potencial de Morse . . . . . . . . . . . . . . . . . . . . . . . . . . . . . . . . . . 13

3.1 .1 Equação de Schrödinger . . . . . . . . . . . . . . . . . . . . . . . . 13

3.1 .2 Descrição Algébrica . . . . . . . . . . . . . . . . . . . . . . . 15

3.2 Potencial de Pöschl-Teller . . . . . . . . . . . . . . . . . . . . . . . 17

3.2 .1 Equação de Schrödinger . . . . . . . . . . . . . . . . . . . . . . . . . 17

3.2 .2 Descrição algébrica . . . . . . . . . . . . . . . . . . . . . . . . 18

3.3 O método algébrico para moléculas diatômicas . . . . . . . . . . . . . . . . . . . 19

3.3 .1 Espectro Vibracional . . . . . . . . . . . . . . . . . . . . . . . . . . . . . 19

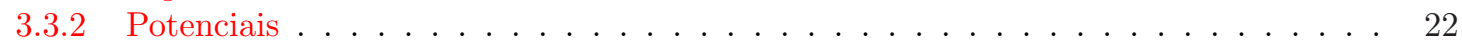

3.3 .3 Fatores de Franck-Condon . . . . . . . . . . . . . . . . . . . . . 25

$3.4 \mathrm{O}$ método algébrico para moléculas poliatômicas $\ldots \ldots \ldots \ldots \ldots \ldots$

4 Resultados e Discussões $\quad 29$

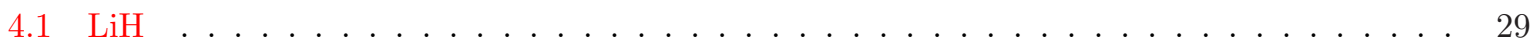

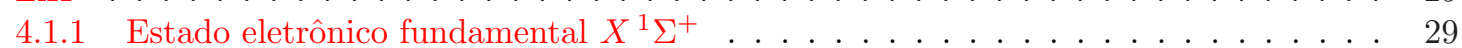

4.1.2 Primeiro estado eletrônico excitado $A^{1} \Sigma^{+} \ldots \ldots \ldots \ldots$. . . . . . . . 32

4.1.3 Fatores de Frank-Condon entre os estados $A-X^{1} \Sigma^{+} \ldots \ldots \ldots$. . . . . . . 33

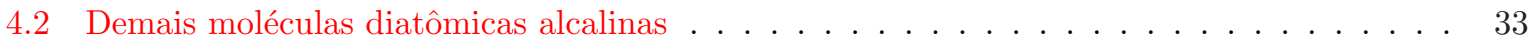

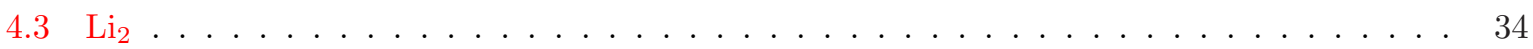

5 Conclusões e perspectivas $\quad 53$

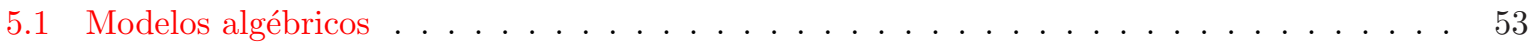

5.1 .1 Modelos com deformação $(q \neq 1) \ldots \ldots \ldots \ldots \ldots \ldots \ldots$

5.1 .2 Caso sem deformação $(q=1) \ldots \ldots \ldots \ldots \ldots \ldots \ldots$

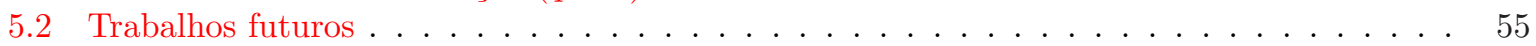

$\begin{array}{ll}\text { A Valores médios de } J_{x} \text { e } J_{y} & \mathbf{5 7}\end{array}$

$\begin{array}{ll}\text { B Realização de Morse para a álgebra su(2) } & 61\end{array}$ 


\section{Lista de Tabelas}

3.1 Constantes usadas em três descrições para o potencial de Morse . . . . . . . . . . . . . . 27

4.1 Constantes moleculares recomendadas para os isotopômeros da molécula LiH . . . . . . . 34

4.2 Parâmetros da hamiltoniana efetiva para o estado $X^{1} \Sigma^{+}$dos isotopômeros da molécula LiH 35

4.3 Constantes de Dunham recomendadas e calculadas algebricamente para o estado eletrônico $X^{1} \Sigma^{+}$dos isotopômeros da molécula $\mathrm{LiH} \ldots \ldots$. . . . . . . . . . . . . . . . 35

4.4 Reescalonamento das constantes de Dunham com as massas reduzidas dos isotopômeros da molécula $\mathrm{LiH} \ldots \ldots . \ldots \ldots$. . . . . . . . . . . . . . . . 36

4.5 Ajustes na profundidade dos potenciais algébricos para o estado eletrônico fundamental dos isotopômeros da molécula $\mathrm{LiH}$. . . . . . . . . . . . . . . . . . . . 36

4.6 Parâmetros da hamiltoniana efetiva para o estado $A^{1} \Sigma^{+}$dos isotopômeros da molécula LiH 37

4.7 Constantes de Dunham recomendadas e calculadas algebricamente para o estado eletrônico $A^{1} \Sigma^{+}$dos isotopômeros da molécula LiH . . . . . . . . . . . . . . . . . . 37

4.8 Ajustes na profundidade dos potenciais algébricos para o primeiro estado eletrônico excitado dos isotopômeros da molécula $\mathrm{LiH} \ldots$. . . . . . . . . . . . . . . . 38 


\section{Lista de Figuras}

3.1 Potenciais vibracionais obtidos via estados coerentes . . . . . . . . . . . . . . . . . . . 27

3.2 Valores médios das quatro primeiras potências de $J_{y}$ e $J_{z}$. . . . . . . . . . . . . . . . . . 28

4.1 Os dois primeiros estados eletrônicos da molécula $\mathrm{LiH}$. . . . . . . . . . . . . . . . 38

4.2 Parâmetro independente $A_{0}$ do hamiltoniano vibracional do estado eletrônico fundamental para os isotopômeros da molécula $\mathrm{LiH} \ldots \ldots$. . . . . . . . . . . . . . . 39

4.3 Desvio médio em função do número de parâmetros para os isotopômeros da molécula $\mathrm{LiH}$ no estado eletrônico fundamental . . . . . . . . . . . . . . . . . . . . . . . 40

4.4 Melhor valor de $j$ em função do número de parâmetros para os isotopômeros da molécula LiH no estado eletrônico fundamental . . . . . . . . . . . . . . . . . . . . . . . . . 40

4.5 Parâmetro independente $A_{0}$ em função do número de parâmetros para os isotopômeros da

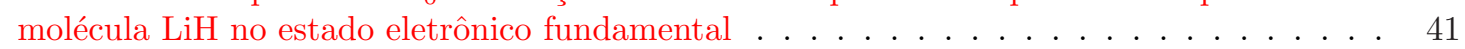

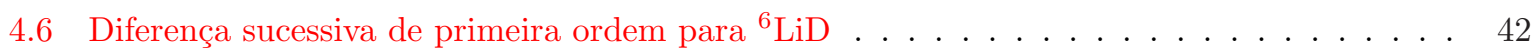

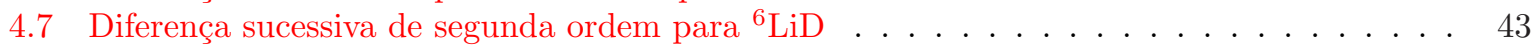

4.8 Diferença sucessiva de terceira ordem para ${ }^{6} \mathrm{LiD}$. . . . . . . . . . . . . . . . . . . . . . 44

4.9 Diferenças sucessivas normalizadas para a molécula ${ }^{7} \mathrm{LiH}$. . . . . . . . . . . . . . . . 44

4.10 Diferenças sucessivas normalizadas para a molécula ${ }^{7} \mathrm{LiD}$. . . . . . . . . . . . . . . . . . . . . . . . . . . 45

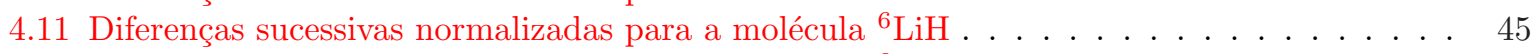

4.12 Diferenças sucessivas normalizadas para a molécula ${ }^{6} \mathrm{LiD}$. . . . . . . . . . . . . . . . 46

4.13 Potenciais para o estado eletrônico fundamental da molécula ${ }^{7} \mathrm{LiH}$. . . . . . . . . . . . . 47

4.14 Potenciais para o primeiro estado eletrônico excitado da molécula ${ }^{7} \mathrm{LiH}$. . . . . . . . . . . 47

4.15 Diferença sucessiva de primeira ordem para os isotopômeros da molécula LiH no primeiro estado eletrônico excitado . . . . . . . . . . . . . . . . . . . . . . . 48

4.16 Diferenças sucessivas normalizadas para a molécula ${ }^{7} \mathrm{LiH}$ no primeiro estado eletrônico



4.17 Fatores de Franck-Condon para os estados eletrônicos $A-X^{1} \Sigma^{+}$da molécula ${ }^{7} \mathrm{LiH}$, bandas $0-0$ e $1-0 \ldots \ldots \ldots \ldots \ldots \ldots$

4.18 Fatores de Franck-Condon para os estados eletrônicos $A-X^{1} \Sigma^{+}$da molécula ${ }^{7} \mathrm{LiH}$, bandas

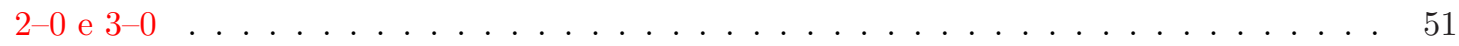




\section{Resumo}

Os fundamentos para os métodos algébricos unidimensionais baseados na álgebra de momentum angular su(2) (clássica e deformada) são apresentados numa forma adequada à descrição de vibrações moleculares de sistemas diatômicos e de sistemas poliatômicos planares com simetria $C_{2 v}$. Aplicações detalhadas envolvendo os estados vibracionais e seus respectivos potenciais foram exaustivamente desenvolvidas para os dois primeiros estados eletrônicos $(A-X \Sigma)$ dos sistemas diatômicos $\mathrm{LiH}$ (e seus isotopômeros), $\mathrm{NaH}, \mathrm{KH}, \mathrm{RbH}, \mathrm{CsH}$, e $\mathrm{Li}_{2}$. Alguns outros estados eletrônicos mais excitados também foram analisados algebricamente. Os potenciais vibracionais semi-clássicos foram calculados via estados coerentes do grupo $\mathrm{SU}(2)$. Calculamos também fatores de Franck-Condon usando as autofunções de Morse diretamente, sem qualquer necessidade de linearizações. O presente trabalho é a análise algébrica mais detalhada até o momento sobre moléculas diatômicas, pois envolve um grande número de moléculas distintas e seus estados eletrônicos excitados, os quais ainda não haviam sido analisados algebricamente. Além disto, outras propriedades físicas, como os fatores de Franck-Condon e potenciais vibracionais foram discutidos aqui em detalhes pela primeira vez. O propósito deste trabalho foi de servir como um laboratório para futuras aplicações de modelos algébricos unidimensionais a sistemas moleculares maiores, onde suas potencialidades e, principalmente, suas limitações pudessem ser testadas de forma clara. Além disto, devemos destacar também o aumento expressivo na quantidade de trabalhos sobre moléculas diatômicas e triatômicas devido à importância destas em questões de interesse mundial, como o efeito estufa, e questões de relevância acadêmica, como a espectroscopia em tempo real e de moléculas frias. 


\begin{abstract}
This is a critical review of one-dimensional algebraic methods applied to molecular spectroscopy of diatomic molecules. We discuss in detaisl two algebraic models based on the angular momentum algebra $\mathrm{su}(2)$ and address several important issues to future works. The applications are done with the alkali hydrides $\mathrm{LiH}, \mathrm{NaH}, \mathrm{KH}, \mathrm{RbH}, \mathrm{CsH}$ and the symmetric molecule $\mathrm{Li}_{2}$. In these applications, we have calculated vibrational energies, vibrational potencials and Franck-Condon factors. We believe this review was necessary to establish the qualities and the limitations of those one-dimensional algebraic models since we have in mind its application to tri-atomic and tetra-atomic planar (non-linear) molecules. Besides that, there is a huge amount of work beeing done with diatomic and triatomic molecules because of their importance in the greenhouse effect, real time spectroscopy and cold molecules spectroscopy.
\end{abstract}




\section{Capítulo 1}

\section{Introdução}

Este trabalho contém uma revisão crítica, bem como resultados novos, dos aspectos principais sobre modelos algébricos unidimensionais construídos para descrever vibrações moleculares [1, 2, 3, 4]. O termo algébrico significa que álgebras de Lie $[5,6,7,8,9]$, convenientemente escolhidas, serão usadas para gerar espectros moleculares de vibração. É bem conhecido que dispomos de vários métodos comprovadamente eficazes, os quais denominaremos de métodos ab initio, para descrever espectros eletrônicos de sistemas moleculares, incluindo vibrações e rotações. Então qual é o objetivo de desenvolvermos um método alternativo baseado em álgebras de Lie? Acontece que as álgebras de Lie, descobertas em 1895 por M. Sophus Lie [10] e, independentemente, por W. Killing, foram "redescobertas" por volta de 1960, principalmente devido aos trabalhos pioneiros de E. Wigner [11] e H. Weyl [12, 13], como instrumentos indispensáveis à organização das propriedades conhecidas de partículas elementares, como massas e energias [14]. As álgebras de Lie mostraram-se também muito úteis no desenvolvimento de um modelo único [15] para a física das partículas elementares (ou das altas energias), conhecido hoje como "modelo padrão" [16, 17, 18, 19]. Assim, nasceu a idéia de usar álgebras de Lie para gerar espectros (de massa, energias, etc.) [20, 21]. Esta idéia foi rapidamente utilizada, com muito sucesso, em física nuclear, onde vibrações nucleares são descritas através de álgebras de Lie [22, 23, 24]. Finalmente, na década de 80, estudos sobre a possibilidade de usarmos álgebras de Lie para gerar espectros moleculares foram iniciados [25, 24]. Estes estudos são conhecidos hoje como modelos algébricos para a espectroscopia molecular [26].

Como mencionado no início, estaremos interessados aqui em modelos algébricos unidimensionais construídos com a álgebra de momentum angular su(2). Embora, originalmente, a álgebra maior su(4) tenha sido utilizada nas primeiras abordagens algébricas [25], principalmente envolvendo moléculas lineares, há pelo menos dois bons motivos para o desenvolvimento de modelos algébricos unidimensionais com base na álgebra de momentum angular: (1) a álgebra de momentum angular su(2) é de conhecimento amplo e muito mais fácil de ser manipulada que a álgebra su(4); (2) muitos sistemas moleculares (diatômicos e planares) possuem potenciais moleculares com um comportamento típico de potenciais unidimensionais exatamente solúveis como Morse e/ou Pöschl-Teller, os quais podem ser descritos algebricamente pela álgebra su(2) [27, 28, 29]. Além destes dois fatos, podemos acrescentar também uma dificuldade técnica na utilização da álgebra su(4) em sistemas moleculares não-lineares: o número de graus de liberdade algébricos é sempre maior que o número de modos normais fundamentais. Também mencionamos nosso interesse apenas na parte vibracional do espectro eletrônico molecular. Tendo em vista que é sempre observada uma hierarquia nas escalas das energias envolvidas nestes espectros moleculares, onde as transições rotacionais ocorrem dentro de cada uma das transições vibracionais, as quais ocorrem dentro de cada transição eletrônica, e que cada uma destas três partes diferem por um fator 10 em suas respectivas energias, podemos muito bem tratar cada parte separadamente, numa primeira aproximação, principalmente no que diz respeito à parte vibracional. Do ponto de vista de modelos teóricos, esta separabilidade é justificada pela aproximação de núcleos fixos (Born-Oppenheimer), a qual é confirmada experimentalmente para o estado eletrônico fundamental. Portanto, restringir os modelos algébricos à parte vibracional é um início indispensável para uma melhor compreensão destes modelos. Posteriormente, uma descrição algébrica da parte rotacional e seus acoplamentos com a parte vibracional, como o acoplamento devido a forças de Coriolis, podem ser efetuadas. 
Em essência, o processo de geração de espectros moleculares por álgebras su(2) (ou por qualquer outra álgebra de Lie) pode ser resumido da forma seguinte. Uma álgebra $\mathrm{su}(2)$ é associado a cada modo normal de vibração. Naturalmente, estamos admitindo aqui que os modos normais possam ser descritos por potenciais unidimensionais. Caso contrário outras álgebras deverão ser utilizadas (por exemplo, $\mathrm{su}(3)$ para potenciais bidimensionais e $\mathrm{su}(4)$ para potenciais tridimensionais). Assim, numa molécula diatômica, precisaremos apenas de uma álgebra $\mathrm{su}(2)$; de três álgebras $\mathrm{su}(2)$ numa molécula tri-atômica; de seis álgebras su(2) numa molécula tetra-atômica planar; etc. Nestes modelos algébricos, as interações entre as ligações químicas serão descritas matematicamente por somas diretas entre as diversas álgebras de Lie. Como conseqüência destas somas diretas, teremos uma álgebra maior cujos geradores servirão para a construção de um hamiltoniano algébrico efetivo. Este hamiltoniano efetivo será diagonalizado, sem dificuldades, no espaço portador das representações irredutíveis desta álgebra maior. Isto é perfeitamente possível, pois a teoria de representação para todas as álgebras de Lie é muito bem conhecida $[6,7,8,9]$. Isto significa que os modos de vibração estarão associados com os vetores de uma determinada representação irredutível de uma determinada álgebra de Lie. Naturalmente, o hamiltoniano efetivo conterá parâmetros arbitrários que deverão ser determinados fenomenologicamente, por exemplo, comparando as energias observadas e calculadas algebricamente. Felizmente ou infelizmente, não existe qualquer "receita" específica para a construção do melhor hamiltoniano efetivo. Cada termo do hamiltoniano algébrico deve ser "desenhado" para reproduzir da melhor forma os detalhes observados em um determinado espectro vibracional (ou ro-vibracional). Tendo as energias vibracionais otimizadas de forma acurada e as correspondentes funções de onda, então é preciso ajustar estas funções de onda para reproduzir propriedades físicas. O teste mais simples, porém muito eficaz, é o cálculo dos fatores de Franck-Condon (superposição de funções de onda vibracionais em estados eletrônicos diferentes) [1, 30]. A inversão do espectro vibracional, isto é, a determinação de potenciais vibracionais a partir destes hamiltonianos efetivos acurados, é outro teste importante. Neste trabalho, estaremos preocupados com a determinação de hamiltonianos efetivos acurados, com a determinação dos fatores de Franck-Condon e da obtenção de potenciais vibracionais.

De volta aos objetivos destes modelos, podemos afirmar que não há qualquer pretensão em substituir os métodos ab initio por modelos algébricos. O nosso interesse aqui é duplo. Primeiro, temos o interesse de aprofundar nossos conhecimentos sobre estes modelos. Devido ao crescente avanço das técnicas experimentais em espectroscopia molecular, possibilitando inclusive o acompanhamento de dissociações moleculares em tempo real e a espectroscopia de moléculas frias, vibrações moleculares constitui um excelente ambiente para o desenvolvimento de modelos algébricos. Certamente, os métodos ab initio são indispensáveis nestes estudos. Segundo, queremos construir descrições complementares e/ou alternativas aos modos normais, os quais não são adequados para etiquetar níveis vibracionais em regiões de altas energias, principalmente quando há a presença de interações não-lineares fortes, como ressonâncias de Fermi, por exemplo. Embora as técnicas experimentais atuais permitam uma excelente resolução na obtenção de espectros moleculares, livres de congestionamento rotacional em muitos casos, ainda não dispomos de uma forma mais eficiente do que os modos normais e suas combinações para identificar os modos de vibração na região de energias altas, energias próximas da energia de dissociação), em sistemas poliatômicos. Os modos normais, definidos no regime de energias baixas, tornam-se inadequados no regime de energias altas devido, principalmente, aos diversos efeitos não-lineares nesta região, os quais provocam um forte congestionamento entre as linhas vibracionais. Um caminho para tentar estabelecer novos "números quânticos" é a realização de estudos sobre a dinâmica clássica associada aos hamiltonianos algébricos efetivos [31, 26]. Estudos atuais nesta direção envolvem muitos elementos modernos de métodos matemáticos da física como teoria de bifurcações [32, 33] e propriedades globais dos grupos de Lie [34]. Portanto, estes modelos podem funcionar também como um excelente laboratório para o desenvolvimento de métodos matemáticos da física. Naturalmente, uma colaboração com experimentalistas em física molecular é imprescindível para o completo sucesso destes estudos, por exemplo, como aquela que está sendo feita em Yale [35, 30, 36].

Neste trabalho, trataremos o espectro vibracional de várias moléculas diatômicas da família dos alcalinos. Sendo mais precisos, analisaremos os dois primeiros estados eletrônicos para hidretos alcalinos [37, 38] $\mathrm{LiH}, \mathrm{NaH}, \mathrm{KH}, \mathrm{RbH}, \mathrm{CsH}$ e a molécula simétrica [39, 40, 41, 42] $\mathrm{Li}_{2}$. Estas análises ainda não foram realizadas em nenhum modelo algébrico. Apenas o hidrogênio molecular e seus isotopômeros têm sido estudados através de modelos algébricos [43]. Nos restringiremos aqui ao estudo dos seguintes aspectos: (1) dificuldades com a otimização dos parâmetros livres do modelo. Em geral, há também a necessidade de otimizações não-lineares; (2) cálculo de probabilidades de transição usando diretamente as auto-funções da álgebra su(2), geralmente funções de Legendre ou de Laguerre; (3) obtenção de potenciais vibracionais 
semi-clássicos via estados coerentes; (4) unificar estas informações em um único processo de otimização. Posteriormente, estes estudos serão estendidos a sistemas sistemas moleculares pequenos, planares e com simetria discreta $C_{2 v}$. Sistemas moleculares pequenos significam moléculas com menos de dez átomos. Em particular, estaremos mais interessados em moléculas diatômicas devido à importância destas em questões ambientais como o efeito estufa, diretamente ou como radicais, em espectroscopia de tempo real e de moléculas frias, além da facilidade computacional reduzida em relação a sistemas maiores. A restrição a moléculas planares com $C_{2 v}$ será por comodidade, embora isto não signifique que estes sistemas apresentem espectros vibracionais simples. Em particular, estaremos interessados nas moléculas tri-atômicas [44, 45, 46] $\mathrm{H}_{2} \mathrm{O}, \mathrm{SO}_{2}, \mathrm{NO}_{2}$ (simétrica), etc., e na molécula tetra-atômica $\mathrm{H}_{2} \mathrm{CO}$ e seus derivados [47].

Os capítulos seguintes estão organizadas da seguinte forma: o Seção 2.1 apresenta a definição da álgebra su(2) e suas representações irredutíveis; a Seção 2.3 apresenta os estados coerentes associados à álgebra su(2); as energias e auto-funções do potencial de Morse e de Pöschl-Teller são rederivadas via equação de Schrödinger e algebricamente na Seção 3.1; o programa algébrico para moléculas diatômicas é discutido em detalhes na Seção 3.3; a Seção 3.4 apresenta um resumo do programa algébrico para moléculas planares tri e tetra-atômicas. Uma análise algébrica feita para a molécula diatômica LiH é apresentada em detalhes na Seção 4.1. Nossas conclusões e perspectivas futuras estão contidas no Capítulo 5 . 


\section{Capítulo 2}

\section{Ferramentas Algébricas}

Descreveremos sucintamente neste seção todas as ferramentas algébricas que serão utilizadas na descrição dos níveis vibracionais de moléculas diatômicas e poliatômicas. Estas ferramentas serão construídas com elementos da álgebra de momentum angular $\mathrm{su}(2)$ e da sua generalização $\mathrm{su}_{\mathrm{q}}(2)$, conhecida como álgebra q-deformada (ou grupo quântico). Como a álgebra su(2) pode descrever bem potenciais vibracionais essencialmente unidimensionais, nos limitaremos a moléculas diatômicas e moléculas planares não lineares nesta dissertação. Neste capítulo e nos demais, usaremos ":=” para denotar uma definição.

\subsection{A Álgebra clássica su(2)}

A álgebra de Lie su(2), associada ao grupo de Lie SU(2), na sua forma canônica, pode ser definida pelas seguintes relações de comutação:

$$
\left[J_{+}, J_{-}\right]=2 J_{z}, \quad\left[J_{z}, J_{ \pm}\right]= \pm 2 J_{ \pm}
$$

Esta álgebra é conhecida também como so(3), a "álgebra do momentum angular",

$$
\left[J_{x}, J_{y}\right]=i J_{z}, \quad\left[J_{z}, J_{x}\right]=i J_{y}, \quad\left[J_{y}, J_{z}\right]=i J_{x}
$$

onde

$$
J_{ \pm}:=J_{x} \pm i J_{y}
$$

A álgebra $\mathrm{su}(2)$ tem somente um operador invariante (também conhecido como operador de Casimir, uma homenagem ao físico holandês H. B. G. Casimir, um dos primeiros a usar este operador em física em 1931),

$$
J^{2}:=J_{x}^{2}+J_{y}^{2}+J_{z}^{2}=J_{z}^{2}+\frac{1}{2}\left(J_{+} J_{-}+J_{-} J_{+}\right)
$$

Este operador comuta com todos os elementos da álgebra, $\left[J^{2}, J_{k}\right]=0$. Como mostrado em (2.1), há somente uma subálgebra, formada por $J_{z}$, em su(2): $\mathrm{su}(2) \supset \mathrm{so}(2)$, onde so(2) é a subálgebra (abeliana) gerada por $J_{z}$. Naturalmente, como so(2) tem apenas o elemento $J_{z}$, este é também um operador invariante. Note que o invariante da sub-álgebra so(2) é, ao contrário do invariante da álgebra su(2), linear nos elementos da álgebra. Dependendo da necessidade, tanto $J_{x}$ quanto $J_{y}$ podem ser escolhidos para substituir $J_{z}$ em (2.1).

Cada representação irredutível hermiteana da álgebra su(2) (ou anti-hermiteana, correspondente a uma representação irredutível unitária do grupo $\mathrm{SU}(2)$ ) é etiquetada por um número inteiro (ou semiinteiro) positivo $j$; cada vetor da representação $j$ será denotado por $|j, m\rangle, m \leq|j|$. Assim, cada representação irredutível $j$ tem dimensão $2 j+1$. Os valores inteiros de $j$ são também as representações irredutíveis da álgebra so(3). Note que estamos usando uma notação também utilizada em mecânica quântica $[48,49]$. Os números quânticos $j$ e $m$ estão relacionados aos auto-valores dos operadores invariantes $J^{2}$ da álgebra $\mathrm{su}(2)$ e $J_{z}$ da sub-álgebra so(2). Os elementos de matriz dos invariantes $J^{2}, J_{z}$ e 
dos operadores $J_{ \pm}$são $[48,49,50]$

$$
J^{2}|j, m\rangle=j(j+1)|j, m\rangle, \quad J_{z}|j, m\rangle=m|j, m\rangle, \quad J_{ \pm}|j, m\rangle=\sqrt{(j \mp m)(j \pm m+1)}|j, m \pm 1\rangle .
$$

Devido à forma desta última ação, os operadores $J_{ \pm}$também são conhecidos como operadores levantamento $\left(J_{+}\right)$e abaixamento $\left(J_{-}\right)$. Em particular, a representação fundamental (matrizes irredutíveis de ordem mais baixa) $j=1 / 2$ para os operadores $J_{z}, J_{ \pm}$é

$$
J_{z}=\frac{1}{2}\left(\begin{array}{cc}
1 & 0 \\
0 & -1
\end{array}\right), \quad J_{+}=\left(\begin{array}{cc}
0 & 1 \\
0 & 0
\end{array}\right), \quad J_{-}=\left(\begin{array}{ll}
0 & 0 \\
1 & 0
\end{array}\right)
$$

as quais são proporcionais às matrizes de Pauli [48, 49, 50].

Os operadores $J_{z}$ e $J_{ \pm}$também podem ser realizados por operadores bosônicos $a$ e $b$, os quais formam a álgebra de Weyl (ou Weyl-Heisenberg),

$$
\left[a, a^{\dagger}\right]=\left[b, b^{\dagger}\right]=1, \quad\left[a, b^{\dagger}\right]=\left[b, a^{\dagger}\right]=[a, b]=0 .
$$

Para isto, basta usar as matrizes da representação fundamental (2.6) e a técnica de Schwinger [51]:

$$
\begin{aligned}
& J_{z}=\left(\begin{array}{ll}
a^{\dagger} & b^{\dagger}
\end{array}\right) \frac{1}{2}\left(\begin{array}{cc}
1 & 0 \\
0 & -1
\end{array}\right)\left(\begin{array}{l}
a \\
b
\end{array}\right)=\frac{1}{2}\left(a^{\dagger} a-b^{\dagger} b\right) \\
& J_{+}=\left(\begin{array}{ll}
a^{\dagger} & b^{\dagger}
\end{array}\right)\left(\begin{array}{ll}
0 & 1 \\
0 & 0
\end{array}\right)\left(\begin{array}{l}
a \\
b
\end{array}\right)=a^{\dagger} b \\
& J_{-}=\left(\begin{array}{ll}
a^{\dagger} & b^{\dagger}
\end{array}\right)\left(\begin{array}{ll}
0 & 0 \\
1 & 0
\end{array}\right)\left(\begin{array}{l}
a \\
b
\end{array}\right)=a b^{\dagger} \\
& \mathbb{N}=\left(\begin{array}{ll}
a^{\dagger} & b^{\dagger}
\end{array}\right)\left(\begin{array}{ll}
1 & 0 \\
0 & 1
\end{array}\right)\left(\begin{array}{l}
a \\
b
\end{array}\right)=a^{\dagger} a+b^{\dagger} b,
\end{aligned}
$$

onde o último termo foi adicionado por conveniência. O conjunto $\left\{\mathbb{N}, J_{z}, J_{ \pm}\right\}$é a forma canônica da álgebra $\mathrm{u}(2)=\mathbb{N} \oplus \mathrm{su}(2)$. O operador IN (operador número) é um operador invariante para a álgebra $\mathrm{u}(2)$ e para a álgebra de Weyl (2.7). Os vetores $|j, m\rangle$, definidos em (2.5), podem ser reconstruídos a partir dos operadores bosônicos da álgebra de Weyl (2.7),

$$
\frac{\left(a^{\dagger}\right)^{n_{a}}}{\sqrt{n_{a} !}}|0\rangle=\left|n_{a}\right\rangle \otimes|0\rangle, \quad a|0\rangle:=0, \quad \frac{\left(b^{\dagger}\right)^{n_{b}}}{\sqrt{n_{b} !}}|0\rangle=|0\rangle \otimes\left|n_{b}\right\rangle, \quad b|0\rangle:=0,
$$

onde $|0\rangle$ é o estado de vácuo, definido por $a|0\rangle=0$ e $b|0\rangle=0$. Usando (2.8), encontraremos que os vetores $|j, m\rangle$ estão relacionado com a representação (2.9) da álgebra de Weyl por

$$
|j, m\rangle=C(j, m)\left(a^{\dagger}\right)^{j+m}\left(b^{\dagger}\right)^{j-m}|0\rangle, \quad C(j, m):=\frac{1}{\sqrt{(j+m) !(j-m) !}}
$$

Note que $N:=n_{a}+n_{b}=(j+m)+(j-m)=2 j$ é o número total de bósons. Em particular, a ação do invariante IN no vetor $|j, m\rangle$ é

$$
\mathbb{I N}|j, m\rangle=N|j, m\rangle, \quad N=2 j .
$$

Este resultado também pode ser obtido considerando a relação

$$
J^{2}=\frac{\mathbb{N}}{2}\left(\frac{\mathbb{N}}{2}+1\right)
$$

entre o operador de Casimir $J^{2}$, definido em (2.4), e o invariante linear IN.

Como dito anteriormente, caso um dos operadores

$$
J_{x}=\frac{1}{2}\left(J_{+}+J_{-}\right), \quad J_{y}=\frac{1}{2 i}\left(J_{+}-J_{-}\right)
$$

seja diagonalizado no lugar de $J_{z}$ em (2.5), por exemplo $J_{y}$,

$$
J_{y}\left|j, m_{y}\right\rangle=m_{y}\left|j, m_{y}\right\rangle,
$$

sempre é possível escrever os antigos auto-estados $|j, m\rangle$ de $J_{z}$ em termos dos auto-estados $\left|j, m_{y}\right\rangle$ de $J_{y}$,

$$
|j, m\rangle=\sum_{m_{y}}\left\langle j, m_{y} \mid j, m\right\rangle\left|j, m_{y}\right\rangle
$$


onde

$$
\left\langle j, m_{y} \mid j, m\right\rangle=\frac{(-i)^{m_{y}+m}}{(-2)^{j}} \frac{C\left(j, m_{y}\right)}{C(j, m)} \sum_{k=0}^{j+m_{y}} \sum_{l=0}^{j+m}(-1)^{k}\left(\begin{array}{c}
j+m_{y} \\
k
\end{array}\right)\left(\begin{array}{c}
j-m_{y} \\
l
\end{array}\right) \delta_{k+l, j+m},
$$

com $C\left(j, m_{y}\right)$ sendo a mesma expressão dada em (2.10), e

$$
\left|j, m_{y}\right\rangle=C\left(j, m_{y}\right) 2^{-j}\left(a^{\dagger}+i b^{\dagger}\right)^{j+m_{y}}\left(i a^{\dagger}+b^{\dagger}\right)^{j-m_{y}}|0\rangle .
$$

Os estados $\left|j, m_{y}\right\rangle$ foram escritos em termos dos operadores bosônicos em (2.17) através de uma mudança de base efetuada com a rotação

$$
\mathbb{R}_{x}=\frac{1}{\sqrt{2}}\left(\begin{array}{ll}
1 & i \\
i & 1
\end{array}\right)
$$

em torno do eixo $x$. Quando aplicada ao vetor de operadores bosônicos $\left(a^{\dagger}, b^{\dagger}\right)$, obtém-se os novos operadores bosônicos

$$
\mathbb{R}_{x} a^{\dagger}=\frac{1}{\sqrt{2}}\left(a^{\dagger}+i b^{\dagger}\right), \quad \mathbb{R}_{x} b^{\dagger}=\frac{1}{\sqrt{2}}\left(i a^{\dagger}+b^{\dagger}\right) .
$$

Note também que estas transformações, $\mathbb{R}_{x}$ e $\mathbb{R}_{x}^{*}$ ou, simplesmente, $\mathbb{R}$, efetuam as seguintes trocas entres os elementos da álgebra $\mathrm{su}(2)$ :

$$
\mathbb{R} J_{x}=J_{x}, \quad \mathbb{R} J_{y}=J_{x}, \quad \mathbb{R} J_{z}=J_{y} .
$$

Desta forma, a expressão (2.17) é obtida aplicando-se a transformação (2.18) em (2.10) e com a troca de $m$ por $m_{y}$. Transformações similares podem ser construídas para efetuarem outras trocas, quando desejado.

\section{$2.2 \quad$ A álgebra q-deformada $\mathrm{su}_{\mathrm{q}}(2)$}

As álgebras q-deformadas foram introduzidas recentemente na década de 80 como um caso especial de álgebras de Hopf $[52,53,54]$. Elas dependem de um parâmetro complexo q e são construídas de tal forma que no limite $\mathrm{q}=1$ sejam as álgebras de Lie clássicas. O parâmetro q é conhecido como parâmetro de deformação.

Apesar de terem um papel fundamental em sistemas exatamente integráveis em Mecânica Estatística $[55,56,57,58]$, ainda é desconhecida a sua importância em outros sistemas físicos, principalmente onde a contrapartida clássica é relevante. Várias tentativas de envolver álgebras deformadas em Física Nuclear e Molecular via modelos algébricos não foram conclusivas [59, 56, 60, 61]. Desta forma, também estaremos buscando neste trabalho qualquer evidência da presença de álgebras deformadas em espectroscopia molecular.

Em geral, para se definir uma álgebra q-deformada é necessário introduzir uma q-deformação na álgebra de Lie clássica correspondente e uma estrutura de Hopf [53, 54]. A estrutura de Hopf não é necessária para o manuseio de representações, exceto no caso de haver produtos tensoriais, e não nos deteremos com ela neste trabalho. A deformação envolve os geradores simples na base de Cartan-Weyl (ou de Chevalley) [62]. Em outras palavras, para o presente caso, onde a álgebra clássica é su(2), a relação de comutação entre os operadores $J_{ \pm}$deve ser modificada, conforme descrito a seguir, para que a álgebra deformada $\operatorname{su}_{\mathrm{q}}(2)$ possa ser definida.

Antes de definirmos a álgebra deformada $\mathrm{su}_{\mathrm{q}}(2)$, precisamos definir uma q-deformação de uma quantidade $x$ qualquer:

$$
[x]_{\mathrm{q}}:=\frac{\mathrm{q}^{x}-\mathrm{q}^{-x}}{\mathrm{q}-\mathrm{q}^{-1}}=\frac{\sinh \omega x}{\sinh \omega}, \quad \mathrm{q}=\mathrm{e}^{\omega}, \quad \omega \in \mathbb{C} .
$$

Algumas propriedades são imediatas:

$$
\lim _{q \rightarrow 1}[x]_{\mathrm{q}}=x, \quad[0]_{\mathrm{q}}=0, \quad[1]_{\mathrm{q}}=1, \quad[x]_{1 / \mathrm{q}}=[x]_{\mathrm{q}} .
$$

Outra relação, igualmente importante, entre quantidades q-deformadas é [53]

$$
[A]_{\mathrm{q}}[B-C]_{\mathrm{q}}+[C]_{\mathrm{q}}[A-B]_{\mathrm{q}}+[B]_{\mathrm{q}}[C-A]_{\mathrm{q}}=0 .
$$


Em particular, estaremos interessados também no caso onde o parâmetro de deformação está sob o círculo unitário $\left(q \in C^{1}\right)$. Neste caso,

$$
[x]_{\mathrm{q}}=\frac{\operatorname{sen} \omega x}{\operatorname{sen} \omega}, \quad \mathrm{q}=\mathrm{e}^{i \omega}, \omega \in \mathbb{R} .
$$

Note que a deformação é real, apesar do parâmetro de deformação ser complexo (de módulo 1).

A álgebra deformada $\mathrm{su}_{\mathrm{q}}(2)$ é definida [53] pelas seguintes relações de comutação envolvendo uma deformação:

$$
\begin{aligned}
{\left[\mathcal{J}_{z}, \mathcal{J}_{ \pm}\right] } & = \pm \mathcal{J}_{ \pm} \\
{\left[\mathcal{J}_{+}, \mathcal{J}_{-}\right] } & =\left[2 \mathcal{J}_{z}\right]_{\mathrm{q}}
\end{aligned}
$$

Naturalmente, a álgebra clássica $\mathrm{su}(2)$ é recuperada no limite $\mathrm{q} \rightarrow 1$. Tendo definido a álgebra deformada, temos que estudar suas representações irredutíveis. Em geral, as representações irredutíveis das álgebras deformadas ainda são etiquetadas pelos mesmos números quânticos clássicos. Os respectivos elementos de matrizes

$$
\begin{aligned}
\mathcal{J}^{2}|j, m\rangle & =[j]_{\mathrm{q}}[j+1]_{\mathrm{q}}|j, m\rangle, \\
\mathcal{J}_{z}|j, m\rangle & =m|j, m\rangle, \\
\mathcal{J}_{ \pm}|j, m\rangle & =\sqrt{[j \mp m]_{\mathrm{q}}[j \pm m+1]_{\mathrm{q}}}|j, m \pm 1\rangle,
\end{aligned}
$$

onde

$$
\mathcal{J}^{2}:=\left[\mathcal{J}_{z}\right]_{\mathrm{q}}^{2}+\frac{1}{2}\left(\mathcal{J}_{+} \mathcal{J}_{-}+\mathcal{J}_{-} \mathcal{J}_{+}\right)
$$

também são bem conhecidos $[52,53]$. A novidade aparece apenas quando o parâmetro de deformação q é uma das raízes da unidade em (2.24). Neste caso pode haver perda de irredutibilidade, isto é, alguns elementos de matrizes em (2.27)-(2.29) podem se anular na raiz da unidade, mesmo quando são não-nulos classicamente. Note que, apenas quando $\mathrm{q} \in \mathbb{R}^{+}$ou $\mathrm{q} \in C^{1}$, podemos impor $\mathcal{J}_{z}^{\dagger}=\mathcal{J}_{z}$ e $\mathcal{J}_{ \pm}^{\dagger}=\mathcal{J}_{\mp}$, como é desejado em física, para obtermos elementos de matrizes reais e, conseqüentemente, autovalores reais para os observáveis construídos a partir dos elementos da álgebra $\mathrm{su}_{\mathrm{q}}(2)$. Ou seja, unitariedade pode ser imposta apenas para o caso de termos um parâmetro de deformação real. Vale mencionar também que a representação fundamental de qualquer álgebra de Lie deformada nunca é deformada, isto é, as matrizes fundamentais $(j=1 / 2)$ para os operadores $\left\{\mathcal{J}_{z}, \mathcal{J}_{ \pm}\right\}$também serão as mesmas matrizes calculadas em (2.6).

Embora seja possível realizar a álgebra $\mathrm{su}_{\mathrm{q}}(2)$ por operadores bosônicos q-deformados [53], isto não será feito aqui neste momento. Os principais motivos são os seguintes: (1) não existe uma forma única de definir os operadores q-bosônicos; (2) não iremos calcular propriedades físicas, neste trabalho, usando um modelo algébrico q-deformado, além do espectro vibracional. Posteriormente, iremos precisar da realização bosônica e de seus respectivos estados coerentes, bem como funções de onda q-deformadas [63, $64]$.

\subsection{Estados coerentes para o grupo $\mathrm{SU}(2)$}

Estados coerentes $[65,66,67,68,69]$ constituem um conjunto de ferramentas algébricas igualmente úteis para o desenvolvimento de modelos algébricos por permitirem o cálculo de quantidades clássicas como valores médios de quantidades quânticas representadas pelos elementos de uma álgebra de Lie. Iremos utilizá-los aqui para derivarmos os potenciais vibracionais associados às hamiltonianos algébricos efetivos. Portanto, dentre todas as definições de estados coerentes, usaremos uma ligada diretamente aos grupos de Lie [67].

Os seguintes passos resumem a construção algébrica dos estados coerentes [67]. (1) Precisa-se de uma álgebra de Lie, e seu respectivo grupo, que tenha uma representação irredutível finita (espaço de Hilbert finito). Para o presente caso, temos a álgebra su(2), definida em (2.1), e o seu respectivo grupo SU(2). O espaço de Hilbert, de dimensão $2 j=1$, corresponde às diferentes representações irredutíveis dadas em (2.5). (2) É necessário também escolher um estado de referência no espaço de Hilbert. Em geral, este estado é escolhido como sendo o estado de peso mais baixo (ou de mínima energia). No caso das representações (2.5), o estado de referência será $|j,-j\rangle$, isto é, $m=-j$. (3) Um grupo de isotropia. Como 
o grupo é, em geral, a exponencial da álgebra, alguns elementos do grupo deixam invariante (a menos de uma fase) o estado de referência. O conjunto destes elementos formam o grupo de isotropia, geralmente um subgrupo do grupo de Lie em questão. No presente caso, o único elemento do grupo $\mathrm{SU}(2)$ que deixa o estado de referência $|j,-j\rangle$ invariante é $h:=\mathrm{e}^{\phi J_{z}}$,

$$
\mathrm{e}^{\phi J_{z}}|j,-j\rangle=\mathrm{e}^{-\phi j}|j,-j\rangle
$$

onde estamos usando o elemento $J_{z}$ como o operador invariante da subálgebra so(2). Isto é evidenciado algebricamente pela relações de comutação (2.1). O conhecimento do subgrupo de isotropia nos permite escrever um elemento qualquer $g$ do grupo $\mathrm{SU}(2)$ como

$$
g=\Omega \cdot h, \quad \Omega(\xi):=\mathrm{e}^{\xi J_{+}-\xi^{*} J_{-}}, \quad h:=\mathrm{e}^{\phi J_{z}},
$$

onde $\Omega$ é o espaço coset, isto é, todos os demais elementos que não estão no subgrupo de isotropia. Como $J_{z}$ é o gerador do subgrupo $\mathrm{SO}(2)$ e não comparece na exponencial $\Omega$, o espaço coset é comumente denotado por $\mathrm{SU}(2) / \mathrm{SO}(2)$ (notação de espaços quocientes $[7,8]$ ).

Usando as definições acima, o estado coerente $|j, \xi\rangle$ do grupo $\mathrm{SU}(2)$ é definido pela ação de um elemento do espaço coset $\Omega$ sobre o estado de referência $|j,-j\rangle$,

$$
|j, \xi\rangle:=\Omega(\xi)|j,-j\rangle=\mathrm{e}^{\xi J_{+}-\xi^{*} J_{-}}|j, \xi\rangle .
$$

Note que a definição do subgrupo de isotropia reduz significativamente a escolha do estado de referência neste caso. A única liberdade que temos é escolher $m=j$ (peso mais alto) com outro estado de referência, mas esta escolha produz o mesmo estado coerente (2.33). No entanto, o estado coerente definido na forma (2.33) não é muito útil para calcularmos valores médios de quantidades algébricas, como o hamiltoniano vibracional, por exemplo. Para efetuarmos a exponenciação em (2.33), precisaremos re-escrever a exponencial da soma $\xi J_{+}-\xi^{*} J_{-}$como um produto de exponenciais em cada um dos três elementos da álgebra $\mathrm{su}(2)$,

$$
\mathrm{e}^{\xi J_{+}-\xi^{*} J_{-}}=\mathrm{e}^{\tau J_{+}} \mathrm{e}^{\alpha J_{z}} \mathrm{e}^{-\tau J_{-}}=\mathrm{e}^{-\tau^{*} J_{-}} \mathrm{e}^{-\alpha J_{z}} \mathrm{e}^{\tau J_{+}}
$$

onde

$$
\tau:=\frac{\xi}{|\xi|} \tan |\xi|, \quad \alpha:=\ln \left(1+|\tau|^{2}\right)
$$

As expressões (2.34) são conhecidas como fórmulas de Baker-Campbell-Hausdorff (BCH) [7] e são facilmente derivadas, neste caso, usando a representação fundamental $j=1 / 2$ da álgebra $\operatorname{su}(2)$, calculadas explicitamente em (2.6). Com as expressões (2.34), a exponencial em (2.33) pode ser resolvida,

$$
|j, \xi\rangle=|j, \tau\rangle=\left(1+|\tau|^{2}\right)^{-j} \sum_{m=-j}^{j}\left(\begin{array}{c}
2 j \\
j+m
\end{array}\right)^{\frac{1}{2}} \tau^{j+m}|j, m\rangle .
$$

Desta forma, podemos ver que estes estados coerentes estão determinados por um único parâmetro contínuo (complexo) $\tau$. Vale mencionar que, entre muitas outras propriedades [67] exibidas pelos estados coerentes (2.36), eles não são ortogonais, mas estão normalizados, $\langle j \tau \mid j \tau\rangle=1$.

As matrizes da representação fundamental $j=1 / 2$, calculadas em (2.6), também podem ser usadas para interpretarmos geometricamente o espaço coset $\mathrm{SU}(2) / \mathrm{SO}(2)$,

$$
\Omega(\xi)=\mathrm{e}^{\xi J_{+}-\xi^{*} J_{-}}=\left(\begin{array}{cc}
\cos |\xi| & \frac{\xi}{|\xi|} \operatorname{sen}|\xi| \\
-\frac{\xi^{*}}{|\xi|} \operatorname{sen}|\xi| & \cos |\xi|
\end{array}\right)
$$

Assim, o parâmetro original $\xi$ pode ser escrito como

$$
\xi=\frac{\theta}{2} \mathrm{e}^{-i \phi}, \quad 0 \leq \theta \leq \pi, 0 \leq \phi \leq 2 \pi .
$$

Portanto, podemos interpretar $\Omega(\xi)$ como uma projeção da esfera $\left(S^{2}\right)$ no plano complexo. Para tal, basta colocarmos o centro de uma esfera de diâmetro unitário localizada a um diâmetro acima do plano complexo $x y$ ( $x$ sendo o eixo real e $y$ o eixo imaginário). Um ponto localizado em $(\theta, \phi)$ sobre a esfera 
é localizado no plano complexo pela interseç̧ão da reta que passa pelo centro da esfera e pelo ponto $(\theta / 2, \phi)$ na superfície esférica. Desta geometria e da definição (2.38), ou da relação (2.35), temos

$$
\tau=\tan \frac{\theta}{2} \mathrm{e}^{-i \phi}, \quad 0 \leq \theta \leq \pi, 0 \leq \phi \leq 2 \pi .
$$

Esta interpretação geométrica nos permite verificar facilmente que os estados coerentes (2.36) são supercompletos, isto é, eles também satisfazem a resolução da unidade

$$
(2 j+1) \int d \mu|j, \tau\rangle\left\langle j, \tau\left|=\sum_{m=j}^{-j}\right| j, m\right\rangle\langle j, m|=1, \quad d \mu=\frac{1}{4 \pi} \operatorname{sen} \theta d \theta d \phi .
$$

Como estaremos interessados em análogos clássicos de quantidades definidas em termos dos elementos da álgebra su(2), é conveniente introduzirmos os parâmetros

$$
\tau=\frac{z}{\sqrt{1-|z|^{2}}}, \quad z:=\frac{1}{\sqrt{\omega}}(q+i p), \quad z^{*}=\frac{1}{\sqrt{\omega}}(q-i p), \quad \omega=4 j,
$$

onde $q$ e $p$ são quantidades reais. Note que estamos usando uma letra romana para escrevermos o parâmetro de deformação q e uma letra itálica para a variável canônica q. Estes novos parâmetros permitem a definição de uma estrutura simpléctica [67]

$$
\{A, B\}:=\frac{\partial A}{\partial p} \frac{\partial B}{\partial q}-\frac{\partial A}{\partial p} \frac{\partial B}{\partial q},
$$

onde $A$ e $B$ são funções (operadores) arbitrárias das variáveis canônicas $p$ e $q$. Desta forma, pode ser mostrado [70] que os valores médios $\langle\cdot\rangle$, em relação ao estado coerente $(2.36)$, dos geradores do grupo $\mathrm{SU}(2)$ satisfazem

$$
\left\{\left\langle J_{x}\right\rangle,\left\langle J_{y}\right\rangle\right\}=\left\langle J_{z}\right\rangle \text {, p.c., }\left\langle J_{z}\right\rangle=\left\langle j, \tau\left|J_{z}\right| j, \tau\right\rangle .
$$

Assim, as relações de comutação da álgebra su(2) são preservadas pela forma simpléctica definida em (2.42). Portanto, valores médios calculados com os estados coerentes devem representar quantidades clássicas. Note em (2.41) que

$$
|\tau|^{2}=\frac{|z|^{2}}{1-|z|^{2}} \geq 0 \quad \Rightarrow \quad|z|^{2} \leq 1 \quad \Rightarrow \quad p^{2}+q^{2} \leq \omega .
$$

Assim, usando $\omega=4 j$, o espaço de fase $(p, q)$ é confinado em uma esfera de raio $4 j$, onde $j$ é a etiqueta de uma representação irredutível (de dimensão $2 j+1$ ) da álgebra su(2) e $\mathrm{su}_{\mathrm{q}}(2)$, quando o parâmetro de deformação q não for uma das raízes da unidade.

\subsection{Estados coerentes q-deformados}

Embora existam alguns trabalhos sobre a construção de estados coerentes associados de alguma forma à álgebra deformada $\mathrm{su}_{\mathrm{q}}(2)$, eles não serão discutidos aqui, neste momento. No entanto, em um trabalho futuro, buscaremos construir estados coerentes q-deformados que sejam compatíveis com os hamiltonianos algébricos q-deformados que iremos testar neste trabalho para que potenciais vibracionais q-deformados também sejam testados. Vale mencionar que, nesta direção, os estados coerentes construídos por Quesne e colaboradores [71] são os mais apropriados até o momento. O único inconveniente é a presença de um elemento de volume na decomposição da unidade (relação análoga a (2.40)), expresso em termos de uma q-exponencial, a qual oferece algumas dificuldades ao cálculo numérico de certas integrais.

\subsection{Valores médios}

Em geral, veremos que os hamiltonianos vibracionais construídos algebricamente serão polinômios em $J_{y}$ ou $J_{z}$ ou em ambos. Por isto, iremos calcular explicitamente os valores médios dos três geradores 
$J_{x}, J_{y}$ e $J_{z}$, usando os estados coerentes clássicos obtidos na Seção $2.3 \mathrm{com} J_{z}$ sendo o gerador da subálgebra so(2). Desta forma, esperamos que o valor médio da hamiltoniana algébrica possa nos fornecer potenciais vibracionais. Em geral, estes valores médios apresentam uma dificuldade considerável para serem calculados. Uma opção muito utilizada é uma aproximação [72, 73, 74, 75, 43] em 1/j, conhecida como bósons intensivos [33, 25]. No entanto, decidimos aqui pelo cálculo explícito.

O valor médio mais simples de ser calculado completamente é o valor médio de $J_{z}$, pois os estados coerentes (2.36) já estão escritos nos auto-estados de $J_{z}$. Assim,

$$
\left\langle j \tau\left|J_{z}^{k}\right| j \tau\right\rangle=\left(1+|\tau|^{2}\right)^{-2 j} \sum_{m_{z}=-j}^{j}\left(\begin{array}{c}
2 j \\
j+m
\end{array}\right)\left(|\tau|^{2}\right)^{j+m_{z}} m_{z}^{k} .
$$

Para facilitar a execução da soma anterior, faremos $n:=m_{z}+j$ e $x:=|\tau|^{2}$,

$$
\left\langle j \tau\left|J_{z}^{k}\right| j \tau\right\rangle=(1+x)^{-2 j} \sum_{n=0}^{2 j}\left(\begin{array}{c}
2 j \\
n
\end{array}\right)(n-j)^{k} x^{n} .
$$

Tendo em conta que

$$
\sum_{n=0}^{2 j}\left(\begin{array}{c}
2 j \\
n
\end{array}\right) x^{n}=(1+x)^{2 j}
$$

então, multiplicando a expressão anterior por $x^{-j}$, derivando com respeito a $x$ e multiplicando o resultado por $x$, obtemos

$$
x \frac{d}{d x} \frac{(1+x)^{2 j}}{x^{j}}=x^{-j} \sum_{n=0}^{2 j}\left(\begin{array}{c}
2 j \\
n
\end{array}\right)(n-j)^{1} x^{n} .
$$

Repetindo este procedimento $k$ vezes, obteremos

$$
\sum_{n=0}^{2 j}\left(\begin{array}{c}
2 j \\
n
\end{array}\right)(n-j)^{k} x^{n}=x^{j}\left(x \frac{d}{d x}\right)^{k} \frac{(1+x)^{2 j}}{x^{j}} .
$$

Assim, o valor médio em (2.45) reduz-se à expressão

$$
\left\langle j \tau\left|J_{z}^{k}\right| j \tau\right\rangle=\frac{x^{j}}{(1+x)^{2 j}}\left(x \frac{d}{d x}\right)^{k} \frac{(1+x)^{2 j}}{x^{j}}, \quad x=|\tau|^{2},
$$

a qual é mais adequada à computação algébrica.

Vejamos alguns exemplos. Para $k=0$, podemos observar apenas que os estados coerentes estão normalizados. Para $k=1$, temos

$$
\left\langle J_{z}^{1}\right\rangle=\left\langle j \tau\left|J_{z}^{1}\right| j \tau\right\rangle=-j \frac{1-x}{1+x} \Rightarrow \lim _{j \rightarrow \infty} \frac{1}{j}\left\langle J_{z}^{1}\right\rangle=-\frac{1-x}{1+x} .
$$

O limite $j \rightarrow \infty$ nesta última expressão é conhecido como limite clássico. Para $k=2$, temos

$$
\left\langle J_{z}^{2}\right\rangle=\left\langle j \tau\left|J_{z}^{2}\right| j \tau\right\rangle=2 j \frac{x}{(1+x)^{2}}+j^{2}\left(\frac{1-x}{1+x}\right)^{2} \Rightarrow \lim _{j \rightarrow \infty} \frac{1}{j^{2}}\left\langle J_{z}^{2}\right\rangle=\left(\frac{1-x}{1+x}\right)^{2} .
$$

Note que a quantidade

$$
\left\langle\Delta J_{z}^{2}\right\rangle=\left\langle J_{z}^{2}\right\rangle-\left\langle J_{z}^{1}\right\rangle^{2}=2 j \frac{x}{(1+x)^{2}}
$$

tem um único máximo em $x=1(\theta=\pi / 2)$ uma assíntota em 0 e limite clássico nulo. Esta mesma quantidade para $k=3$,

$$
\left\langle\Delta J_{z}^{3}\right\rangle=\left\langle J_{z}^{3}\right\rangle-\left\langle J_{z}^{1}\right\rangle^{3}=-2 j(3 j-1) \frac{x}{(1+x)^{2}} \frac{1-x}{1+x}
$$

tem um único máximo (mínimo) em $x=2-\sqrt{3}(x=2+\sqrt{3})$ e uma assíntota em 0 . Naturalmente, estaremos interessados nestes valores médios em termos das variáveis canônicas $(q, p)$ definidas em (2.41):

$$
x=\frac{y}{1-y} \Rightarrow 1+x=\frac{1}{1-y}, 1-x=\frac{1-2 y}{1-y}, \quad y=|z|^{2}=\frac{1}{\omega} Q_{+}^{2}, Q_{ \pm}^{2}:=q^{2} \pm p^{2},
$$


onde, por conveniência, estamos usando uma constante arbitrária $\omega$ no lugar da constante $4 j$ aparecendo em (2.41). Os valores médios das duas primeiras potências de $J_{z}$ podem ser re-escritos em termos das variáveis canônicas $(q, p)$ na seguinte forma:

$$
\begin{aligned}
\left\langle J_{z}^{1}\right\rangle & =\frac{2 j}{\omega} Q_{+}^{2}-j, \\
\left\langle J_{z}^{2}\right\rangle & =\frac{2 j(2 j-1)}{\omega^{2}}\left(Q_{+}^{4}-\omega Q_{+}^{2}\right)+j^{2} .
\end{aligned}
$$

Veremos mais adiante, na Seção 3, como certas transformações podem ser construídas para que os potenciais de Morse e de Pöschl-Teller possam ser re-obtidos destes valores médios. Em geral, estas transformações dependerão do número quântico $j$. Isto significa que o limite clássico deve ser re-calculado após tais transformações terem sido estabelecidas.

O cálculo do valor médio dos demais operadores, $J_{x}$ e $J_{y}$, é um pouco mais complicado. Por isto, vamos nos limitar ao cálculo explícito até a potência dois. Conforme mostrado no Apêndice A, os valores médios de $J_{x}$ e $J_{y}$ podem ser re-escritos como:

$$
\left\langle J_{x}\right\rangle=+\frac{2 j}{\omega} q \sqrt{\omega-Q_{+}^{2}}, \quad\left\langle J_{y}\right\rangle=-\frac{2 j}{\omega} p \sqrt{\omega-Q_{+}^{2}},
$$

onde a quantidade $Q_{+}^{2}$ está definida em (2.55), e

$$
\begin{aligned}
& \left\langle J_{x}^{2}\right\rangle=\frac{j}{2}-\frac{2 j(2 j-1)}{\omega^{2}}\left(q^{4}-\omega q^{2}+q^{2} p^{2}\right), \\
& \left\langle J_{y}^{2}\right\rangle=\frac{j}{2}-\frac{2 j(2 j-1)}{\omega^{2}}\left(p^{4}-\omega p^{2}+q^{2} p^{2}\right) .
\end{aligned}
$$

Podemos observar que os valores médios (2.58) e (2.59) estão relacionados, respectivamente, pelas seguintes transformações canônicas: $\{q \rightarrow-p, p \rightarrow-q\}$ e $\{q \rightarrow p, p \rightarrow q\}$. Portando, eles fornecem as mesmas informações físicas. Note também que os valores médios em (2.58) são reais apenas quando a condição (2.44) é satisfeita, isto é, quando $Q_{+}^{2} \leq \omega$. Em geral, a quantidade $\sqrt{\omega-Q_{+}^{2}}$ aparecerá somente nos valores médios das potências ímpares de $J_{x}$ e $J_{y}$. 


\section{Capítulo 3}

\section{Modelo Algébrico Unidimensional}

Neste capítulo, descreveremos em detalhes um modelo algébrico adequado para a descrição de níveis vibracionais de sistemas moleculares poliatômicos, cujos potenciais vibracionais possuam um caráter unidimensional. Em geral, moléculas diatômicas e várias moléculas planares podem ser descritas razoavelmente bem por potenciais unidimensionais. Veremos também que, em muitos casos, a descrição algébrica pode ser considerada acurada, isto é, comparável a métodos ab initio. Iniciaremos expondo a motivação de qualquer modelo algébrico, isto é, os potenciais de Morse e de Pöschl-Teller e suas descrições algébricas. Em seguida apresentaremos o modelo algébrico para moléculas diatômicas e sua extensão a moléculas poliatômicas.

\subsection{Potencial de Morse}

Em 1929, P. Morse propôs o potencial [76]

$$
V(r)=D_{e}\left(\mathrm{e}^{-2 \alpha\left(r-r_{0}\right)}-2 \mathrm{e}^{-\alpha\left(r-r_{0}\right)}\right)
$$

para descrever os níveis de energia vibracionais de moléculas diatômicas, onde $r_{0}$ é a posição de equilíbrio para a distância interatômica, $D_{e}=V\left(r_{0}\right)-V(r \rightarrow \infty)$ é a profundidade do potencial e $\alpha$ é o alcance do potencial. A energia de dissociação é $D_{0}=D_{e}-E(v=0)$, onde $E(v=0)$ é a energia do estado vibracional fundamental.

Este potencial possui todas as características qualitativas requeridas de um potencial diatômico efetivo descrevendo as vibrações do estado eletrônico fundamental: (1) ele tem somente um ponto de mínimo; (2) tende a zero para grandes valores de $r$ e (3) tem um valor muito grande, simulando uma barreira infinita, quando os núcleos estão muito próximos. Além disto, este potencial apresenta soluções analíticas para o caso unidimensional, as quais podem ser interpretadas à luz da teoria das álgebras de Lie [77, 27, 28]. Tudo isto o torna um paradigma para o desenvolvimento de modelos algébricos em Física Molecular. Nas seções seguintes, faremos uma exposição breve da resolução do potencial de Morse unidimensional via equação de Schrödinger e álgebras de Lie.

\subsubsection{Equação de Schrödinger}

Devido à diferença grande entre a massa nuclear e a massa eletrônica em qualquer molécula, é razoável supor que o movimento nuclear seja muito mais lento que o movimento eletrônico. Enquanto esta aproximação é válida para o estado eletrônico fundamental, ela deixa de ser válida para os estados eletrônicos mais excitados. Nesta aproximação, conhecida como aproximação de Born-Oppenheimer, os núcleos de uma molécula diatômica sentem a presença de um potencial efetivo com as características do potencial de Morse (3.1). Desta forma, a equação de Schrödinger para uma molécula diatômica, dentro da aproximação de Born-Oppenheimer, é

$$
H \psi=-\frac{\hbar^{2}}{2 \mu}\left(\frac{\partial^{2}}{\partial r^{2}}+\frac{2}{r} \frac{\partial}{\partial r}+\frac{L^{2}}{\hbar^{2} r^{2}}\right) \Psi+V(r) \psi=E \Psi,
$$


onde $\mu$ é a massa reduzida e $V(r)$ é o potencial de Morse (3.1). Fazendo as substituições usuais

$$
\Psi=Y_{l m}(\theta, \phi) \frac{R(r)}{r}, \quad L^{2} Y_{l m}(\theta, \phi)=-\hbar^{2} l(l+1) Y_{l m}(\theta, \phi),
$$

onde $L^{2}$ é o operador momentum angular,

$$
L^{2}=-\hbar^{2}\left[\frac{\partial^{2}}{\partial \theta^{2}}+\cot \theta \frac{\partial}{\partial \theta}+\frac{1}{\operatorname{sen} \theta} \frac{\partial^{2}}{\partial \phi^{2}}\right]
$$

a equação de Schrödinger (3.2) pode ser re-escrita como

$$
\left[\frac{d^{2}}{d r^{2}}-\frac{2 \mu}{\hbar^{2}} V(r)-\frac{l(l+1)}{r^{2}}\right] R(r)=-\frac{2 \mu}{\hbar^{2}} E R(r) .
$$

Para o caso $l=0$, correspondendo a vibrações sem a presença de rotações, podemos encontrar uma solução analítica para a versão unidimensional da equação (3.5), isto é, considerando $-\infty<r<+\infty$. Para isto, devemos introduzir as seguintes variáveis:

$$
z:=2 \lambda \mathrm{e}^{-2 \alpha\left(r-r_{0}\right)}, \quad u(z)=z^{-\frac{\xi}{2}} \mathrm{e}^{\frac{z}{2}} R(r), \quad \lambda^{2}:=\frac{2 \mu}{(\alpha \hbar)^{2}} D_{e}, \quad \xi^{2}:=-4 \frac{2 \mu}{(\alpha \hbar)^{2}} E .
$$

Desta forma, a nova auto-função $u(z)$ satisfaz a equação de Kummer [78, 79],

$$
\left\{z \frac{d^{2}}{d z^{2}}+(b-z) \frac{d}{d z}+a\right\} u(z)=0, \quad a:=\lambda-\frac{1}{2} b, \quad b:=\xi+1 .
$$

Para que $u$ seja uma solução finita, então é preciso que $a=v$ seja um número inteiro positivo e que $\xi \geq 0$, isto é, a energia em (3.6) deve negativa, correspondendo a estados ligados. Nestas condições, a solução é um polinômio de Laguerre generalizado,

$$
u=L_{v}^{\xi}(z), \quad v=0,1, \ldots,\left[\lambda-\frac{1}{2}\right],
$$

onde $[x]$ significa o menor inteiro próximo de $x$. Como $a=v$, então de (3.7) e (3.6), a energia vibracional do potencial de Morse pode ser escrita na forma

$$
E_{M}(v)=-\frac{(\alpha \hbar)^{2}}{2 \mu}\left(\lambda-\frac{1}{2}-v\right)^{2}=-D_{e}+\alpha \hbar \sqrt{\frac{2 D_{e}}{\mu}}\left(v+\frac{1}{2}\right)-\frac{(\alpha \hbar)^{2}}{2 \mu}\left(v+\frac{1}{2}\right)^{2} .
$$

A energia $E_{M}(v)+D_{e}$ corresponde aos dois primeiros termos, $Y_{10}$ e $Y_{20}$, da tradicional expansão de Dunham para as energias puramente vibracionais,

$$
E_{D}(v)=\sum_{i=1} Y_{i 0}\left(v+\frac{1}{2}\right)^{i}
$$

onde os coeficientes $Y_{l m}$ são determinados ajustando-se esta expressão aos dados experimentais. Quando há isótopos, as constantes de Dunham $Y_{i 0}$ obedecem à relação

$$
r_{i 0}=\frac{Y_{i 0}^{(a)} / Y_{i 0}^{(b)}}{\left(\mu^{(b)} / \mu^{(a)}\right)^{\frac{i}{2}}}
$$

onde $\mu$ é a massa reduzida dos isótopos $(a)$ e $(b)$.

Assim, os níveis vibracionais do potencial de Morse fornecem a primeira correção anarmônica observada no espectro vibracional experimental. Em geral, é observado também anarmonicidades de ordens mais altas, as quais requerem potenciais mais realistas. Em muitos casos, é necessário usar oito ou dez termos na expansão de Dunham. No entanto, esta situação é muito diferente em moléculas poliatômicas, incluindo moléculas lineares, onde termos quadráticos numa expansão análoga à expansão de Dunham produzem resultados acurados em muitos casos, no que diz respeito aos modos normais das vibrações longitudinais (stretchings). 


\subsubsection{Descrição Algébrica}

Apresentaremos aqui uma descrição algébrica dos níveis de energia vibracional do potencial de Morse, a qual é adequada ao desenvolvimento de modelos algébricos unidimensionais para vibrações moleculares em geral. Esta descrição algébrica está baseada no trabalho de Alhassid et al. [27]. Quando este trabalho já estava terminado, encontramos uma outra descrição algébrica também muito adequada aos nossos propósitos, feita por Dong et al. [29].

O ponto de partida desta descrição algébrica é o mapeamento entre os operadores bosônicos e um espaço de coordenadas. Para fazermos a conexão com o espaço de coordenadas, precisamos primeiro escrever os operadores bosônicos (2.7) em suas formas canônicas

$$
a=\frac{x+i p_{x}}{\sqrt{2}}, \quad a^{\dagger}=\frac{x-i p_{x}}{\sqrt{2}}, \quad b=\frac{y+i p_{y}}{\sqrt{2}}, \quad b^{\dagger}=\frac{y-i p_{y}}{\sqrt{2}}, \quad p_{k}=\frac{\hbar}{i} \frac{\partial}{\partial x_{k}} .
$$

A equação de Schrödinger para o potencial de Morse está associada aos operadores invariantes IN e $J_{y}$. Isto significa que estamos fazendo uso da cadeia de álgebras u(2) つ so(2). Substituindo (3.12) nas definições (2.8), teremos

$$
\mathbb{N}=\frac{1}{2}\left(x^{2}+y^{2}-\frac{\partial^{2}}{\partial x^{2}}-\frac{\partial^{2}}{\partial y^{2}}-2\right), \quad J_{y}=-\frac{i}{2}\left(x \frac{\partial}{\partial y}-y \frac{\partial}{\partial x}\right) .
$$

Note que estamos usando $\hbar=1$ por comodidade. Fazendo uma nova mudança de variáveis da forma

$$
x:=\rho \cos \phi, \quad y:=\rho \operatorname{sen} \phi,
$$

obtemos

$$
\mathbb{N}=\frac{1}{2}\left(\rho^{2}-\frac{1}{\rho} \frac{\partial}{\partial \rho} \rho \frac{\partial}{\partial \rho}-\frac{1}{\rho^{2}} \frac{\partial^{2}}{\partial \phi^{2}}\right), \quad J_{y}=-\frac{i}{2} \frac{\partial}{\partial \phi} .
$$

O próximo passo é a resolução das equações de autovalores para IN e $J_{y}$,

$$
\mathbb{N} \psi=N \psi, \quad J_{y} \psi=m_{y} \psi .
$$

Observando a forma de $J_{y}$ em (3.15), o gerador das rotações em torno do eixo $y$, podemos propor uma solução da forma

$$
\psi=R(\rho) \Phi(\phi), \quad \Phi(\phi):=\mathrm{e}^{-2 i m_{y} \phi} .
$$

Assim, a equação de Morse é obtida com a seguinte mudança de variável:

$$
\rho^{2}:=(N+1) \mathrm{e}^{-\beta}, \quad \beta:=\alpha\left(r-r_{0}\right) .
$$

Desta forma, a equação diferencial para IN torna-se em

$$
\left\{\frac{d^{2}}{d \beta^{2}}-\left(\frac{N+1}{2}\right)^{2}\left(\mathrm{e}^{-2 \beta}-2 \mathrm{e}^{-\beta}\right)\right\} R_{N, m_{y}}(\beta)=m_{y}^{2} R_{N, m_{y}}(\beta) .
$$

Comparando (após uma introdução de unidades apropriadas) este resultado com a equação de Schrödinger (3.5), com o potencial de Morse (3.1) e as definições em (3.6), teremos

$$
D_{e}=\frac{(\hbar \alpha)^{2}}{2 \mu}\left(\frac{N+1}{2}\right)^{2}, \quad \lambda=\frac{N+1}{2}, \quad E_{m_{y}}=-\frac{(\hbar \alpha)^{2}}{2 \mu} m_{y}^{2}, \quad \xi=2 m_{y} \geq 0 .
$$

Note que a última expressão restringe os possíveis valores de $m_{y}(j+1$ valores $)$ e a penúltima expressão nos fornece uma relação entre o número quântico $v$ usado em espectroscopia molecular e os números quânticos $\left(j, m_{y}\right)$ usados em teoria de grupos, se impusermos que esta energia deva ser igual à energia do potencial de Morse em (3.9):

$$
E_{V}=-\frac{(\hbar \alpha)^{2}}{2 \mu} m_{y}^{2}=-\frac{(\alpha \hbar)^{2}}{2 \mu}\left(\lambda-v-\frac{1}{2}\right)^{2}=E_{M} \quad \Rightarrow \quad m_{y}=\frac{N}{2}-v=j-v, \quad N=2 j .
$$

Como $v$ deve ser um inteiro positivo menor ou igual a $\lambda-1 / 2=j$, teremos $j+1$ estados vibracionais. Destes resultados, concluímos que o hamiltoniano vibracional algébrico

$$
H=-\frac{(\hbar \alpha)^{2}}{2 \mu} J_{y}^{2}
$$


tem os mesmos níveis de energia e as mesmas auto-funções do potencial de Morse unidimensional. A Tabela 3.1 resume as relações entre as diversas constantes e variáveis usadas nas duas descrições do potencial de Morse feitas aqui. Por comodidade, incluímos também uma relação com as constantes e variáveis usadas por Matsumoto [79].

Vale mencionar que não temos outra escolha a não ser diagonalizar simultaneamente $J_{y}, J^{2}$ e IN em (3.16), devido à forma específica dos operadores diferenciais em (2.8), caso estejamos interessados somente em re-obter o potencial de Morse. A diagonalização do operador $J_{z}$, ao invés de $J_{y}$, fornece uma descrição algébrica [27] para o potencial de Pöschl-Teller, o qual descreve outro tipo de vibração molecular (bendings). Note também a presença necessária, pelo menos neste procedimento, do operador invariante IN nesta descrição algébrica do potencial de Morse. No entanto, este operador é também um dos elementos da álgebra $\mathrm{u}(2)$, como observado na Seção 2.1. Portanto, o operador IN não pertence à álgebra $\mathrm{su}(2)$ e nem pode ser escrito como um polinômio em seus elementos. Isto significa que esta descrição algébrica é feita com a cadeia $\mathrm{u}(2) \supset \mathrm{so}(1)$. No entanto, o espectro vibracional depende apenas de $J_{y}$, um dos elementos da álgebra su(2) e o único gerador da subálgebra so(2).

Do ponto de vista da teoria de representações, para completar, resta mostrar que as funções de onda

$$
\Psi_{j, m_{y}}(z, \phi)=\Phi_{m_{y}}(\phi) R_{j m_{y}}(z), \quad z=(2 j+1) \mathrm{e}^{-\alpha\left(r-r_{0}\right)}, \quad \Phi_{m_{y}}(\phi)=\mathrm{e}^{-2 i m_{y} \phi}
$$

onde a parte radial são as auto-funções de Morse

$$
R_{j m_{y}}(z)=A_{j m_{y}} z^{m_{y}} \mathrm{e}^{-z / 2} L_{j-m_{y}}^{2 m_{y}}(z), \quad A_{j m_{y}}^{2}=2 m_{y} \frac{\left(j-m_{y}\right) !}{\left(j+m_{y}\right) !}
$$

comportam-se de fato como bases para a representação irredutível (2.5). A constante de normalização $A_{j m_{y}}$ em (3.24) é determinada usando-se as relações de ortogonalidade das funções de Laguerre (veja também [79]):

$$
\int_{0}^{\infty} \frac{d z}{z} R_{j m_{y}}(z) R_{j m_{y}^{\prime}}(z)=\delta_{m_{y}, m_{y}^{\prime}}
$$

Deve ser alertado que a medida correta a ser usada em integrais que não envolvem o quadrado das funções de Morse é $d z /(\alpha z)$ e, conseqüentemente, a constante de normalização deve incluir também o alcance $\alpha$ em seu denominador. Note também que a constante de normalização em (3.24) é proporcional a $m_{y}$. Então o estado vibracional correspondendo a $v=j$, o estado mais excitado, terá uma função de onda nula. Portanto, teremos de fato, apenas $j$ estados vibracionais associados às funções de Morse (3.24) na descrição algébrica.

Para mostrarmos que as funções (3.23) comportam-se com auto-estados de $J_{y}$ e seus respectivos operadores de levantamento e abaixamento, iremos re-escrever todos os operadores bosônicos aparecendo nos elementos (2.8) da álgebra $\mathrm{su}(2)$ na forma diferencial $(3.15)(\hbar=1)$. Após a transformação de coordenadas (3.14) e

$$
z=\rho^{2}
$$

os operadores bosônicos podem ser re-escritos como

$$
\begin{aligned}
a & =\sqrt{\frac{z}{2}}\left(\cos \phi+\cos \phi \partial_{z}-z^{-1} \operatorname{sen} \phi \partial_{\phi}\right), & b & =\sqrt{\frac{z}{2}}\left(\operatorname{sen} \phi+\operatorname{sen} \phi \partial_{z}+z^{-1} \cos \phi \partial_{\phi}\right), \\
a^{\dagger} & =\sqrt{\frac{z}{2}}\left(\cos \phi-\cos \phi \partial_{z}+z^{-1} \operatorname{sen} \phi \partial_{\phi}\right), & b^{\dagger} & =\sqrt{\frac{z}{2}}\left(\operatorname{sen} \phi-\operatorname{sen} \phi \partial_{z}-z^{-1} \cos \phi \partial_{\phi}\right) .
\end{aligned}
$$

Um pouco de cuidado deve ser tomado em relação aos elementos da álgebra su(2) quando escritos na forma polar. Como não estamos mais usando $J_{z}$ como o operador diagonal, mas $J_{y}$, então devemos usar

$$
J_{ \pm}^{\prime}=J_{z} \pm i J_{x} \quad \Rightarrow \quad\left[J_{y}, J_{ \pm}^{\prime}\right]= \pm J_{ \pm}^{\prime}, \quad\left[J_{+}^{\prime}, J_{-}^{\prime}\right]=2 J_{y} .
$$

Assim, substituindo as expressões (3.27) nas realizações bosônicas (2.8) e em (2.3), após algumas manipulações algébricas, os novos geradores em (3.28) podem ser realizados por

$$
J_{y}=-\frac{i}{2} \frac{\partial}{\partial \phi}, \quad J_{ \pm}^{\prime}=\frac{\mathrm{e}^{ \pm 2 i \phi}}{4 z}\left[z^{2}+\frac{\partial^{2}}{\partial \phi^{2}}-4 z^{2} \frac{\partial^{2}}{\partial z^{2}} \pm 2 i\left(\frac{\partial}{\partial \phi}-2 z \frac{\partial^{2}}{\partial z \partial \phi}\right)\right] .
$$


Pode ser mostrado que (3.29) é de fato uma realização diferencial para a álgebra su(2), onde os elementos de matriz dos operadores $\left\{J_{y}, J_{ \pm}^{\prime}\right\}$ são aqueles mesmos em (2.5) com $m_{z}$ trocado por $m_{y}$. Como esta verificação não é uma tarefa fácil, omitiremos seus detalhes neste momento, reservando-os para o Apêndice B. No entanto, vale mencionar que esta verificação depende fortemente nas seguintes relações de recorrência para as funções de Laguerre $L=L(x)$ :

$$
\frac{d L_{n}^{\alpha}}{d z}=-L_{n-1}^{\alpha+1}, \quad \frac{d L_{n}^{\alpha}}{d z}=\frac{n L_{n}^{\alpha}-(n+\alpha) L_{n-1}^{\alpha}}{z},
$$

e

$$
L_{n}^{\alpha}=L_{n}^{\alpha+1}-L_{n-1}^{\alpha+1}, \quad x L_{n}^{\alpha}=(2 n+\alpha+1) L_{n}^{\alpha}-(n+1) L_{n+1}^{\alpha}-(n+\alpha) L_{n-1}^{\alpha} .
$$

Embora os resultados (3.29) não estejam presentes na Ref. [27], e nem comentados, deve ser enfatizado que eles são muito diferentes dos operadores diferenciais obtidos em [77], os quais atuam em estados de diferentes representações, portanto, totalmente inadequados para o presente modelo algébrico.

\subsection{Potencial de Pöschl-Teller}

Como afirmamos na introdução, além de moléculas diatômicas, estaremos interessados também em moléculas poliatômicas planares. Moléculas planares, como a água $\left(\mathrm{H}_{2} \mathrm{O}\right)$, exibem também modos vibracionais de deformação angular (bending) além dos modos vibracionais de estiramento (stretching). No caso da água, há dois modos de estiramento, entre os átomos de hidrogênio e oxigênio, e um modo de deformação angular entre as duas ligações HO. A molécula de água em seu estado eletrônico fundamental possui uma simetria $\mathrm{C}_{2 v}$, pois as duas ligações HO são idênticas. Neste caso, $\alpha x=a\left(\theta-\theta_{0}\right)$ é uma coordenada interna adequada para descrever as vibrações envolvendo deformações angulares em torno da posição de equilíbrio $\theta_{0}$. O potencial associado a estes modos deve ser simétrico, $V(x)=V(-x)$, para moléculas com simetria $\mathrm{C}_{2 v}$. Caso as duas ligações químicas formando o modo normal de deformação angular sejam diferentes, uma versão não-simétrica deve ser usada. Isto explica nosso interesse em moléculas planares com simetria $\mathrm{C}_{2 v}$. Portanto, o potencial de Morse não é adequado para descrever deformações angulares. O potencial para o modo normal de deformação angular deve ter também um alcance muito limitado, isto é, ir a zero rapidamente em torno da posição de equilíbrio $x=0$, pois, em geral, estes modos possuem baixa amplitude. Naturalmente, este potencial deve ter uma profundidade compatível com a quantidade de estados ligados existente em cada sistema. Um potencial unidimensional exatamente solúvel com estas características foi proposto em 1933 por Pöschl e Teller [80]:

$$
V(x)=-D_{e} \frac{1}{\cosh ^{2}(\alpha x)},-\infty<x<\infty, \quad D_{e}=\frac{(\alpha \hbar)^{2}}{2 \mu} \lambda(\lambda-1), \quad \lambda>1 .
$$

Veremos a seguir que este potencial admite estados ligados e que seu espectro é idêntico ao níveis de energia do potencial de Morse. Veremos também que estes níveis de energia podem ser re-obtidos facilmente de forma algébrica, usando a álgebra $\mathrm{su}(2)$.

\subsubsection{Equação de Schrödinger}

Sem perda de generalidades, podemos ir diretamente para a equação de Schrödinger unidimensional sem a parte angular, uma vez que não estamos interessados nas rotações $(l=0)$,

$$
\left[\frac{d^{2}}{d \beta^{2}}+\frac{\lambda(\lambda-1)}{\cosh ^{2}(\beta)}-\xi^{2}\right] R^{\prime}(\beta)=0, \quad \beta=\alpha x, \quad \xi^{2}=-\frac{2 \mu}{(\alpha \hbar)^{2}} E .
$$

Tradicionalmente, uma série de substituições é feita para transformar esta equação numa equação diferencial hipergeométrica. A primeira mudança de variável é

$$
y:=\cosh ^{2}(\beta), \quad 1 \leq y<\infty,
$$

a qual torna (3.33) em

$$
\left\{y(1-y) \frac{d^{2}}{d y^{2}}+\left(\frac{1}{2}-y\right) \frac{d}{d y}-\frac{1}{4}\left[\frac{\lambda(\lambda-1)}{y}-\xi^{2}\right]\right\} R^{\prime}(y)=0 .
$$


Agora, a transformação

$$
R^{\prime}(y):=y^{\lambda / 2} R(y)
$$

torna $(3.35)$ em

$$
\left\{y(1-y) \frac{d^{2}}{d y^{2}}+\left[\left(\lambda+\frac{1}{2}\right)-(\lambda+1) y\right] \frac{d}{d y}-\frac{1}{4}\left(\lambda^{2}-\xi^{2}\right)\right\} R(y)=0 .
$$

Esta equação é muito parecida com a equação hipergeométrica, não fosse o fato do intervalo da variável $y$ estar limitado em 1 . Resta então apenas a mudança de variável

$$
z:=1-y, \quad-\infty<z \leq 0 .
$$

Assim, a equação (3.37) torna-se em

$$
\left\{z(1-z) \frac{d^{2}}{d z^{2}}+\left[\frac{1}{2}-(\lambda+1) z\right] \frac{d}{d z}-\frac{1}{4}\left(\lambda^{2}-\xi^{2}\right)\right\} R(z)=0,
$$

a qual é uma equação diferencial hipergeométrica [81] da forma

$$
\left\{z(1-z) \frac{d^{2}}{d z^{2}}+[c-(a+b+1) z] \frac{d}{d z}-a b\right\} R(z)=0
$$

com

$$
a=\frac{1}{2}(\lambda+\xi), \quad b=\frac{1}{2}(\lambda-\xi), \quad c=\frac{1}{2} .
$$

A equação diferencial hipergeométrica (3.39) admite soluções polinomiais [82, 81] quando $2 b$ for um inteiro positivo não nulo, menor ou igual a $\lambda-1$,

$$
2 b=\lambda-\xi=v+1 \quad \Rightarrow \quad E_{P T}(v)=\frac{(\alpha \hbar)^{2}}{2 \mu}(\lambda-1+v)^{2}, \quad v=0,1, \ldots,[\lambda-1] .
$$

Nestas condições, as soluções de (3.39) são séries hipergeométricas finitas, as quais estão relacionadas com as funções de Legendre [82]. Esta relação é feita com muito mais facilidade na descrição algébrica a seguir. No entanto, observe que o espectro vibracional em (3.42) é idêntico àquele do potencial de Morse obtido em (3.9).

\subsubsection{Descrição algébrica}

A descrição algébrica dos níveis de energia do potencial de Pöschl-Teller é muito mais simples do que a descrição algébrica do potencial de Morse [27]. Novamente, consideraremos a cadeia su(2) $\supset \operatorname{so}(2)$.

A realização diferencial dos operadores $\left\{J_{z}, J_{ \pm}\right\}$adequada ao potencial de Pöschl-Teller é a própria definição do momentum angular em Mecânica Quântica [48] $(\hbar=1)$, escrito em coordenadas esféricas,

$$
\begin{aligned}
J_{z} & =-i \frac{\partial}{\partial \phi} \\
J_{ \pm} & =e^{ \pm \phi}\left( \pm \frac{\partial}{\partial \theta} i \cot \phi \frac{\partial}{\partial \phi}\right) \\
J^{2} & =-\left[\frac{1}{\operatorname{sen} \theta} \frac{\partial}{\partial \theta} \operatorname{sen} \theta \frac{\partial}{\partial \theta}+\frac{1}{\operatorname{sen}^{2} \theta} \frac{\partial^{2}}{\partial \phi^{2}}\right]
\end{aligned}
$$

Desta forma, as auto-funções simultâneas de $J_{z}$ e $J^{2}$,

$$
J_{z} Y_{j m}=m Y_{j m}, \quad J^{2} Y_{j m}=j(j+1) Y_{j m},
$$

serão os harmônicos esféricos $Y_{j m}$ [83], com $j$ inteiros,

$$
Y_{j m}(\theta, \phi)=\frac{(-1)^{m}}{\sqrt{2 \pi}} \mathrm{e}^{i m \phi} R_{j m}(\theta), \quad R_{j m}(\theta)=\left[\frac{(2 j+1)}{2} \frac{(j-m) !}{(j+m) !}\right]^{1 / 2} P_{j, m}(\cos \theta),
$$


onde $P_{j, m}(\cos \theta)$ são as funções de Legendre. As funções $R_{j m}(\theta)$ estão normalizadas,

$$
\int_{0}^{\pi} d \theta \operatorname{sen} \theta R_{j, m} R_{j^{\prime}, m}=\delta_{j j^{\prime}}
$$

e obedecem a equação diferencial proveniente da ação do operador de Casimir $J^{2}$ em $Y_{j m}$,

$$
\left[\frac{d^{2}}{d \theta^{2}}+\cot \theta \frac{d}{d \theta}-j(j+1)-\frac{m^{2}}{\operatorname{sen}^{2} \theta}\right] R(\theta)=0 .
$$

Fazendo a substituição [27]

$$
\cos \theta=\tanh \beta, \quad-\infty<\beta<\infty,
$$

a equação (3.47) torna-se na equação diferencial (3.33) para o potencial de Pöschl-Teller,

$$
\left[\frac{d^{2}}{d \beta^{2}}+\frac{j(j+1)}{\cosh ^{2}(\beta)}-m^{2}\right] R(\beta)=0 .
$$

Comparando (3.33) e (3.49), após a introdução das constantes apropriadas, concluímos que

$$
E_{V}=-\frac{(\alpha \hbar)^{2}}{2 \mu} m^{2}=\frac{(\alpha \hbar)^{2}}{2 \mu}(\lambda-1-v)^{2}, \quad \lambda=j+1 \quad \Rightarrow \quad m=j-v .
$$

Observe que o espectro algébrico (3.50) do potencial de Pöschl-Teller é o mesmo espectro algébrico (3.21) do potencial de Morse. Neste caso, diz-se que estes dois potenciais são iso-espectrais [27, 28]. Isto significa que as funções de onda de Morse (3.24) e de Legendre (3.45) estão relacionadas [27]. Note também que teremos $j+1$ estados neste caso e que a profundidade do potencial de Pöschl-Teller também está ligada à representação irredutível $j$.

\subsection{O método algébrico para moléculas diatômicas}

Nesta seção, aplicaremos todos os conceitos algébricos apresentados nas últimas seções para resolver o espectro vibracional de moléculas diatômicas e, a partir dele, calcular outras propriedades físicas. O programa deste modelo algébrico unidimensional pode ser resumido pelos seguintes passos:

1. Construir um hamiltoniano efetivo que seja uma função analítica nos elementos da álgebra su(2), tal que, quando diagonalizado, a expansão de Dunham seja re-obtida.

2. Diagonalizar este hamiltoniano efetivo nas representações irredutíveis da álgebra su(2).

3. Otimizar os parâmetros livres, usando dados experimentais.

4. Calcular o valor médio deste hamiltoniano efetivo usando os estados coerentes do grupo $S U(2)$ associado à álgebra su(2) utilizada.

5. Cálculo dos fatores de Franck-Condon (FC) e de outras propriedades físicas.

Desta forma, moléculas diatômicas servirão como um laboratório para aprendermos o que é possível realizar com este modelo e, principalmente, quais serão suas limitações quando o mesmo for aplicado a moléculas maiores. Embora existam na literatura[72, 73, 84, 85, 43, 30, 25, 24] muitas variantes do programa algébrico que iremos implementar aqui para moléculas diatômicas, não iremos comentá-las neste momento. Mas vale mencionar que estamos muito próximos do modelo utilizado por Cooper e Gupta [43].

\subsubsection{Espectro Vibracional}

Um espectro molecular está dividido em três partes (em ordem decrescente de energia): espectro eletrônico, o espectro vibracional e o espectro rotacional $[1,2]$. Cada um deles tem um intervalo de energia em diferentes escalas e podem ser tratados de forma independente em muitos casos, quando não há 
acoplamentos fortes devido a quase-degenerescências e efeitos não-lineares como ressonâncias. Neste trabalho estudaremos somente o espectro vibracional e somente do ponto de vista algébrico.

Motivados pelas descrições algébricas dos níveis vibracionais dos potenciais unidimensionais de Morse e Pöschl-Teller (Seções 3.1.2 e 3.2.2, respectivamente), construiremos um hamiltoniano algébrico que reproduza o espectro de um potencial de Morse (iso-espectral ao potencial de Pöschl-Teller) em ordem mais baixa, e reproduza a expansão de Dunham (3.10) em ordens mais altas. Dois hamiltonianos efetivos possíveis, satisfazendo os requisitos acima, incluindo também a possibilidade de q-deformações, são da forma

$$
\begin{aligned}
& H_{1}=\sum_{i=0}^{n} A_{i}\left[J_{k}\right]_{\mathrm{q}}^{i}, \quad k \in\{x, y, z\}, \\
& H_{2}=\sum_{i=0}^{n} B_{i}\left[J_{k}\right]_{\mathrm{q}}^{2 i},
\end{aligned}
$$

onde $A_{i}$ e $B_{i}$ são parâmetros lineares a serem determinados pelo método de mínimos quadrados e $j$ e q são os parâmetros não-lineares. Para moléculas diatômicas, estes parâmetros não-lineares podem ser determinados por inspeção. Até onde sabemos, esta hamiltoniana com q $=1$ e incluindo todas as potências em $J_{z}$, o operador invariante da sub-álgebra so(2), foi utilizada somente por Cooper e Gupta [43]. Os demais modelos unidimensionais usam apenas as potências pares de $J_{z}$, tentando imitar uma série de potências em termos de um potencial de Morse.

Os hamiltonianos (3.51)-(3.52) são facilmente diagonalizados na base $\left|J, m_{k}\right\rangle$, conforme mostrado em (2.5) e (2.27)-(2.29),

$$
\begin{aligned}
& E_{1 m_{k}}=\sum_{i=0}^{n} A_{i}\left[m_{k}\right]_{\mathrm{q}}^{i}, \\
& E_{2 m_{k}}=\sum_{i=0}^{n} B_{i}\left[m_{k}\right]_{\mathrm{q}}^{2 i} .
\end{aligned}
$$

Tendo em conta a relação (3.20) entre $m_{k}$ e $v, m_{k}=j-v$, vemos que os espectros vibracionais (3.53) e (3.54) tornam-se em um polinômio no número quântico espectroscópico $v$, similar à expansão de Dunham $(3.10)$,

$$
E_{V}(v)=\sum_{i=0}^{n} A_{i}[j-v]_{\mathrm{q}}^{i}, \quad v=0,1, \ldots, j,
$$

$\mathrm{e}$

$$
E_{V}(v)=\sum_{i=0}^{n} B_{i}[j-v]_{\mathrm{q}}^{2 i}
$$

Note que este espectro vibracional é o mesmo quando qualquer um dos três geradores $J_{x}, J_{y}$ ou $J_{z}$ for usado para ser diagonalizado simultaneamente com o operador de Casimir $J^{2}$. O processo de otimização dos parâmetros livres é explicado a seguir usando (3.55) como exemplo.

Os parâmetros lineares podem, neste caso, ser determinados pelo método dos mínimos quadráticos. A expressão que se deseja otimizar é o desvio médio quadrático

$$
r m s=\frac{1}{N_{e}-N_{p}} \sqrt{\sum_{v=0}^{N_{e}-1}\left[E_{V}(v)-E_{E}(v)\right]^{2}}
$$

onde $E_{V}$ é a energia vibracional algébrica (3.55) ou (3.56), $E_{E}$ é a energia observada (experimental), $N_{e}$ é o número de energias vibracionais observadas e $N_{p}$ é o número total de parâmetros. Por comodidade, re-escreveremos as energias vibracionais (3.55) na forma

$$
E_{V}(v)=\sum_{k=1}^{n} A_{k} \xi_{k}(v), \quad \xi_{k}(v)=[j-v]_{\mathrm{q}}^{k} .
$$

Assim, o mínimo da expressão (3.57), em função dos parâmetros não-lineares $j$ e q, ocorre quando

$$
\frac{\partial r m s}{\partial A_{k}}=0 .
$$


Esta condição conduz ao seguinte conjunto de equações lineares:

$$
\sum_{i=1}^{n} \sum_{v=0}^{N_{e}} \xi_{k}(v) \xi_{i}(v) A_{i}=\sum_{v=0}^{N_{e}} \xi_{k}(v) E_{E}(v),
$$

as quais podem ser re-escritas na forma matricial

$$
\Xi A=\mathcal{E} \quad \Rightarrow \quad A=\Xi^{-1} \mathcal{E}
$$

onde

$$
\Xi_{i k}:=\sum_{v=0}^{N_{e}-1} \xi_{i}(v) \xi_{k}(v), \quad \mathcal{E}_{k}:=\sum_{v=0}^{N_{e}-1} \xi_{k}(v) E_{E}(v) .
$$

Desta forma os parâmetros lineares podem ser calculados invertendo-se a matriz simétrica $\Xi$ em (3.61). Resultados análogos são obtidos para as energias vibracionais (3.56).

Vamos considerar inicialmente o caso clássico $(\mathrm{q}=1)$. Vamos supor a existência de um espectro vibracional com apenas quatro $\left(N_{e}=3\right)$ energias observadas $E_{E}(v)=E_{v}, v=0, \ldots, 3$. Com estas quatro energias observadas, podemos otimizar a expressão (3.55) com $n=2$. Por comodidade, vamos desconsiderarmos o termo $N_{e}-N_{p}$ presente no desvio médio (3.57). Então, neste caso, as matrizes $\mathcal{E}$ e $\Xi$, definidas em (3.62), são:

$$
\begin{aligned}
& \mathcal{E}=\left(\begin{array}{c}
\varepsilon \\
j \varepsilon-\tau \\
j^{2} \varepsilon-(2 j-1) \tau+2\left(E_{2}+3 E_{3}\right)
\end{array}\right), \quad \varepsilon:=\sum_{i=0}^{3} E_{i}, \quad \tau:=\sum_{i=1}^{3} i E_{i}, \\
& \Xi=\left(\begin{array}{ccc}
4 & 4 j-6 \\
4 j-6 & 4 j^{2}-12 j+14 & 4 j^{2}-12 j+14 \\
4 j^{2}-12 j+14 & 4 j^{3}-18 j^{2}+42 j-36 & 4 j^{4}-24 j^{3}+84 j^{2}-144 j+98
\end{array}\right) .
\end{aligned}
$$

As constantes lineares podem então ser calculadas com o auxílio de (3.61):

$$
\begin{aligned}
& A_{0}=\frac{1}{20}\left(\alpha_{3}-\alpha_{2} j+5 \alpha_{1} j^{2}\right), \\
& A_{1}=\frac{1}{20}\left(\alpha_{2}-10 \alpha_{1} j\right), \\
& A_{2}=\frac{1}{4} \alpha_{1}
\end{aligned}
$$

onde

$$
\begin{aligned}
& \alpha_{1}=E_{0}-E_{1}-E_{2}+E_{3}, \\
& \alpha_{2}=21 E_{0}-13 E_{1}-17 E_{2}+9 E_{3}, \\
& \alpha_{3}=19 E_{0}+3 E_{1}-3 E_{2}+E_{3} .
\end{aligned}
$$

Note em (3.65) a seguinte regra para a dependência do número quântico $j$ nas constantes lineares $A_{i}$ : cada constante $A_{i}$ é um polinômio em $j$ de grau $n-i$. Esta é uma regra geral quando incluímos todas as potências em (3.55), para o caso q $=1$. Veremos na Seção 4.1 que o valor da constante $A_{0}$ estará muito próximo da energia de dissociação de uma molécula diatômica. Substituindo as constantes lineares (3.65) na expressão do desvio médio (3.57) ao quadrado, sem o termo $N_{e}-N_{p}$, teremos

$$
r m s^{2}=\frac{1}{20}\left(E_{0}-3 E_{1}+3 E_{2}-E_{3}\right)^{2}=\frac{1}{20}\left(\Delta_{2}^{(3)}\right)^{2},
$$

onde $\Delta_{i}^{(k)}$ são as sucessivas diferenças entre as diferenças sucessivas entre as energias vibracionais,

$$
\Delta_{i}^{(k)}=\Delta_{i+1}^{(k)}-\Delta_{i}^{(k)}, \quad \Delta_{i}^{(1)}=E_{i+1}-E_{i} .
$$

Portanto, o desvio médio quadrático mínimo não depende do parâmetro não-linear $j$. Este é outro resultado geral quando incluímos todas as potências em (3.55), para o caso q $=1$. Desta forma, $j$ está livre para ser usado para ajustar a profundidade do potencial vibracional, ou seja, para garantir que haja aproximadamente $j+1$ estados vibracionais, como previstos experimentalmente. Como $j$ é um número inteiro positivo, este ajuste nem sempre é acurado. Então, um outro parâmetro contínuo, independente 
de $j$, é necessário para que um ajuste fino seja feito. Este poderia ser o papel do parâmetro de deformação q. No entanto, paga-se um preço: o mínimo do desvio médio quadrático passa a depender de q e também de $j$ para o caso $q \neq 1$.

Em geral, usar somente as potências pares como em (3.56) para o caso clássico, q = 1, faz com que o desvio mínimo e os parâmetros lineares dependam também dos parâmetros não-lineares. Esta mesma dependência também é observada no caso $q \neq 1$, mesmo incluindo todas as potências. Naturalmente, nestas duas situações, os parâmetros lineares serão calculados em função dos parâmetros não-lineares $j$ (inteiro e positivo) e q (real ou uma fase) usando (3.61). Neste caso, o valor de $j$, a etiqueta da representação irredutível, deve ser calculado inspecionando-se os valores mínimos do desvio médio quadrático correspondentes a cada valor inteiro de $j$. Além deste critério, procuraremos ter os valores de $j$ que estejam próximos do número $j+1$ de estados vibracionais esperados experimentalmente. Um procedimento geral será iniciar o processo de otimização com um modelo clássico $(q=1)$, onde o desvio médio e as constantes lineares não dependem de $j$. O valor de $j$ será escolhido de forma a termos os $j+1$ estados vibracionais previstos experimentalmente. Como $j$ é um inteiro, a energia de dissociação poderá não ser calculada acuradamente. Para corrigir isto, usaremos a versão deformada $q \neq 1$ com o mesmo valor de $j$ para o caso não-deformado. Assim, esperamos que o parâmetro de deformação possa funcionar como uma sintonia fina para o espectro vibracional algébrico.

A Seção 4.1 contém resultados de vários estudos detalhados do processo de otimização descrito acima e suas variantes, aplicado aos diversos estados eletrônicos da molécula diatômica LiH.

\subsubsection{Potenciais}

Suponha que exista uma molécula diatômica cujo potencial vibracional seja exatamente o potencial de Morse (3.1). Vimos na Seção 3.1.2 como descrever algebricamente este potencial vibracional unidimensional, usando o gerador $J_{y}^{2}$. Esta descrição é possível se a profundidade $D_{e}$ do potencial de Morse estiver relacionada com a etiqueta $j$ de uma representação irredutível (unitária) da álgebra su(2) como em (3.20), onde $D_{e} \sim(2 j+1)^{2} / 4$, ou seja,

$$
V_{M}=-D_{e}\left(2-\mathrm{e}^{-\beta}\right) \mathrm{e}^{-\beta}=-\frac{\eta}{4}[2(2 j+1)-z] z, \quad z=(2 j+1) \mathrm{e}^{-\beta},
$$

onde

$$
\beta=\alpha\left(r-r_{0}\right), \quad D_{e}=\frac{\eta}{4}(2 j+1)^{2}, \quad \eta=\frac{(\hbar \alpha)^{2}}{2 \mu} .
$$

Vimos também na Seção 2.3 que valores médios calculados com os estados coerentes reproduzem quantidades clássicas. Assim, devemos verificar se o valor médio do quadrado de $J_{y}^{2},\left\langle J_{y}^{2}\right\rangle$, ou o quadrado do valor médio de $J_{y},\left\langle J_{y}^{1}\right\rangle^{2}$, calculados na Seção 2.5, reproduzem o potencial de Morse quando a variável canônica associada ao momentum for igualada a zero $(p=0)$. Para completar, faremos também um estudo comparativo entre os valores médios de $J_{y}^{2}$ e $J_{z}^{2}$.

Como veremos logo abaixo, os termos independentes de coordenadas nos valores médios (2.56)-(2.59) podem ser ignorados, pois eles produziram apenas um deslocamento vertical dos potenciais. A fim de usarmos a mesma variável $p$ para representar o momentum linear conjugado à posição ${ }^{1} q$, devemos aplicar as transformações canônicas $\{q \rightarrow \pm p, p \rightarrow \pm q\}$ aos valores médios de $J_{y}$. Após o limite $p=0$ $\left(Q_{ \pm}=q\right)$ ser aplicado, caso queiramos preservar a forma canônica (2.42), os valores médios que estamos interessados podem ser escritos como:

$$
\begin{aligned}
& \left\langle J_{y}^{1}\right\rangle=\mp \frac{2 j}{\omega} q \sqrt{\omega-q^{2}}=\mp \frac{1}{2} q \sqrt{4 j-q^{2}}, \quad\left\langle J_{y}^{1}\right\rangle^{2}=\frac{1}{4}\left[2(2 j)-q^{2}\right] q^{2}, \\
& \left\langle J_{z}^{1}\right\rangle=+\frac{2 j}{\omega} q^{2}=+\frac{1}{2} q^{2}, \quad\left\langle J_{z}^{1}\right\rangle^{2}=\frac{1}{4} q^{4} \\
& \left\langle J_{y}^{2}\right\rangle=+\frac{2 j(2 j-1)}{\omega^{2}}\left(\omega q^{2}-q^{4}\right)=+\frac{(2 j)(2 j-1)}{16 j^{2}}\left[2(2 j) q^{2}-q^{4}\right] \\
& \left\langle J_{z}^{2}\right\rangle=-\frac{2 j(2 j-1)}{\omega^{2}}\left(\omega q^{2}-q^{4}\right)=-\frac{(2 j)(2 j-1)}{16 j^{2}}\left[2(2 j) q^{2}-q^{4}\right],
\end{aligned}
$$

\footnotetext{
${ }^{1}$ Nesta seção, a letra $q$ representa uma variável canônica (posição ou momentum) e não deve ser confundida com o parâmetro de deformação q usado no contexto de álgebras deformadas.
} 
onde $0 \leq q^{2} \leq \omega$ e $\omega=4 j$. Comparando estes resultados com (3.69), vemos claramente que $\left\langle J_{z}^{1}\right\rangle^{2}$ não serve para reproduzir o potencial de Morse. Também observamos que $\left\langle J_{y}^{2}\right\rangle$ e $\left\langle J_{z}^{2}\right\rangle$ fornecem as mesmas informações. Vamos considerar primeiro o valor médio $\left\langle J_{y}^{1}\right\rangle^{2}$. Comparando (3.69) e (3.71), percebemos que a melhor solução deve ter a forma

$$
q^{2}=\theta(j) \mathrm{e}^{-\beta}, \quad \beta=\alpha\left(r-r_{0}\right),
$$

onde $\theta$ é uma função arbitrária de $j$, assim como $\omega$, se não estivermos preocupados em preservar a forma canônica (2.42). Na verdade, a função $\theta$ não é totalmente arbitrária, pois ela deve ser positiva e menor que $\omega$ para que a condição (2.44) seja satisfeita. Então, devemos procurar determinar $\theta$ e $\omega$ que satisfaçam a condição

$$
A_{2}\left\langle J_{y}^{1}\right\rangle^{2}=A_{2}(2 j)^{2}\left(\frac{\theta}{\omega}\right)^{2}\left(\frac{\omega}{\theta}-\mathrm{e}^{-\beta}\right) \mathrm{e}^{-\beta}=-\frac{\eta}{4}(2 j+1)^{2}\left(2-\mathrm{e}^{-\beta}\right) \mathrm{e}^{-\beta}=V_{M},
$$

onde $V_{M}$ é o potencial de Morse em (3.69) e $A_{2}$ é uma constante arbitrária para acertar as unidades. Infelizmente, há nenhum valor de $\theta$ e $\omega$ que satisfaça a condição desejada em (3.76) para qualquer valor de $j$. O melhor que podemos fazer é escolher $\theta=\omega / 2$ e $A_{2}=-\eta$. Assim, ficamos livres para escolher $\omega=4 j$, como em (2.41). Como conseqüência, o valor médio $\left\langle J_{y}^{1}\right\rangle^{2}$ reproduz um potencial de Morse com uma profundidade $D_{e}^{\prime}$ ligeiramente reduzida,

$$
D_{e}^{\prime}=\frac{\eta}{4}(2 j)^{2}=D_{e}-\frac{4 j+1}{4} \approx D_{e}-j .
$$

Em geral, $j \approx 100$ (adimensional) para a maioria dos sistemas moleculares descritos algebricamente. Isto significa uma diferença muito pequena na escala das energias vibracionais (alguns milhares em $\mathrm{cm}^{-1}$. No entanto, esta diferença pode aumentar significativamente quando tivermos que usar uma hamiltoniana contendo potências mais elevadas de $J_{y}$. Uma alternativa é otimizar a função $\theta(j)$ a fim de aproximarmos o máximo da profundidade prevista experimentalmente. Por exemplo, se aplicarmos a transformação $j \rightarrow j+1 / 2$ em $D_{e}^{\prime}$ na Eq. (3.77), obteremos então exatamente o a profundidade do potencial de Morse, obtido com $\left\langle J_{y}^{1}\right\rangle^{2}$. Esperamos também que, no futuro, alguma versão deformada dos estados coerentes clássicos possa corrigir esta deficiência, ou seja, que o parâmetro de deformação possa funcionar uma sintonia fina para a energia de dissociação.

Uma discussão similar à anterior pode ser feita em relação ao valor médio $\left\langle J_{y}^{2}\right\rangle$ :

$$
B_{1}\left\langle J_{y}^{2}\right\rangle=B_{1}(2 j)(2 j-1)\left(\frac{\theta}{\omega}\right)^{2}\left[\frac{\omega}{\theta}-\mathrm{e}^{-\beta}\right] \mathrm{e}^{-\beta}=-\frac{\eta}{4}(2 j+1)^{2}\left(2-\mathrm{e}^{-\beta}\right) \mathrm{e}^{-\beta}=V_{M},
$$

Também neste caso, a melhor escolha para a função $\theta$ em (3.75) continua sendo $\theta=\omega / 2$,

$$
D_{e}^{\prime}=\frac{2 j(2 j-1)}{4}=D_{e}-\frac{6 j+1}{4} \approx D_{e}-\frac{3}{2} j .
$$

Note que neste caso, não há uma transformação simples, como $j \rightarrow j+1 / 2$, que possa ser aplicada em $D_{e}^{\prime}$ para obtermos a profundidade correta do potencial de Morse, obtido com $\left\langle J_{y}^{2}\right\rangle$.

Há pelo menos três maneiras pelas quais as discussões acima podem ser generalizadas e testadas, a fim de que o melhor potencial vibracional diatômico possa ser extraído dos hamiltonianos vibracionais (3.51)-(3.52) usando os estados coerentes clássicos do grupo SU(2) (sem deformação). Uma generalização imediata é considerar o valor médio dos hamiltonianos vibracionais efetivos (3.51)-(3.52) no limite $p=0$,

$$
V(q)=\lim _{p=0}\langle j \tau|H| j \tau\rangle,
$$

onde a coordenada $q$ é relacionada com a distância interatômica $r$ através da transformação de Morse em (3.75). Como veremos logo em seguida, esta generalização parece ser pouco eficaz, independentemente da escolha da função $\theta$. Isto será confirmado mais adiante na Seção 4.1. As outras duas generalizações serão mais eficazes:

$$
\begin{aligned}
& V_{1}(q)=\sum_{i=0}^{n} A_{i}\left\langle J_{y}^{1}\right\rangle^{i}=\sum_{i=0}^{n} A_{i} \theta_{1}^{i}\left[\left(2-e^{-\beta}\right) e^{-\beta}\right]^{\frac{i}{2}}, \quad \theta_{1}=j \rightarrow j+\frac{1}{2}, \\
& V_{2}(q)=\sum_{i=0}^{n} B_{i}\left\langle J_{y}^{2}\right\rangle^{i}=\sum_{i=0}^{n} B_{i} \theta_{2}^{i}\left[\left(2-e^{-\beta}\right) e^{-\beta}\right]^{i}, \quad \theta_{2}=\frac{2 j(2 j-1)}{4} \rightarrow(j+1)^{2},
\end{aligned}
$$


onde fizemos a escolha $\theta=2 j$ em (3.75). As constantes $A$ e $B$ são as mesmas dos hamiltonianos vibracionais (3.51), incluindo todas as potências de $J_{y}$, e (3.52), incluindo somente as potências pares de $J_{y}^{2}$. As funções $\theta_{1}$ e $\theta_{2}$, originalmente $j$ e $2 j(2 j-1) / 4$, respectivamente, funcionam como ajustes finos. Estes dois potenciais têm uma outra característica interessante: ambos apresentam um único mínimo em $\beta=0$, ou seja, em $r=r_{0}$. Note então que os potenciais (3.81) e (3.82) são adequados para descrever somente potenciais vibracionais que apresentam apenas um mínimo isolado. Note também em (3.81) que $2-e^{-\beta} \geq 0$. Isto implica em um valor mínimo para a distância internuclear, $\beta \geq-\ln 2$ ou, equivalentemente, $r \geq r_{0}-\ln 2 / \alpha$. Esta restrição não ocorre nos valores médios das potências pares de $J_{y}$, como pode ser visto em (3.82). As constantes $\alpha$ e $r_{0}$ também devem ser determinadas por um processo de otimização. No entanto, a posição de equilíbrio $r_{0}$ é calculada acuradamente por métodos ab initio.

Vale ressaltar que os valores médios das potências lineares ou maiores que três dos operadores $J_{k}$, $k \in\{x, y, z\}$ não fornecem os mesmos potenciais, ao contrário das potências quadráticas, conforme podemos verificar observando os quatro primeiros termos listados abaixo:

$$
\begin{gathered}
\left\langle J_{y}^{0}\right\rangle=1, \\
\left\langle J_{y}^{1}\right\rangle=-\frac{1}{2} q \sqrt{4 j-q^{2}}, \\
\left\langle J_{y}^{2}\right\rangle=-\frac{(2 j-1)}{8 j} q^{4}+\frac{(2 j-1)}{2} q^{2}, \quad\left\langle J_{z}^{2}\right\rangle=\frac{(2 j-1)}{8 j} q^{4}-\frac{(2 j-1)}{2} q^{2}, \\
\left\langle J_{y}^{3}\right\rangle=\frac{(j-1)(2 j-1)}{16 j^{2}} q^{5} \sqrt{4 j-q^{2}}-\frac{(j-1)(2 j-1)}{4 j} q^{3} \sqrt{4 j-q^{2}}-\frac{(3 j-1)}{4} q \sqrt{4 j-q^{2}}, \\
\left\langle J_{z}^{3}\right\rangle=\frac{(j-1)(2 j-1)}{16 j^{2}} q^{6}-\frac{3(j-1)(2 j-1)}{8 j} q^{4}+\frac{\left(3 j^{2}-3 j+1\right)}{2} q^{2}, \\
\left\langle J_{y}^{4}\right\rangle=\frac{(j-1)(2 j-1)(2 j-3)}{64 j^{3}} q^{8}-\frac{(j-1)(2 j-1)(2 j-3)}{8 j^{2}} q^{6}+\frac{(2 j-1)\left(4 j^{2}-13 j+8\right)}{8 j} q^{4} \\
\left\langle J_{z}^{4}\right\rangle=\frac{(j-1)(2 j-1)(2 j-3)}{64 j^{3}} q^{8}-\frac{(j-1)(2 j-1)(2 j-3)}{8 j^{2}} q^{6}+\frac{(2 j-1)\left(6 j^{2}-12 j+7\right)}{8 j} q^{4} \\
-\frac{(2 j-1)\left(2 j^{2}-2 j+1\right)}{2} q^{2} .
\end{gathered}
$$

Estes valores médios foram calculados diretamente de (2.50) e (A.9) (veja também (A.10)), usando as transformações (2.41) e (A.12), e o limite $p=0$. As constantes aditivas independentes de $q$ também foram removidas. Estas constantes devem ser removidos para que o potencial não seja transladado verticalmente, ou seja, a remoção destes termos faz com o potencial $V(q)-A_{0}$ se anule em $q=0$ (dissociação, $r \rightarrow \infty$ ). Isto significa que a constante linear $A_{0}$ em (3.51) deveria ser interpretada como a profundidade $D_{e}$ do potencial vibracional. No entanto, veremos que esta constante estará mais próxima da energia de dissociação $D_{0}=D_{e}-E_{V}(0)$.

Em geral, quão diferente serão estes potenciais? Observando os valores médios (3.83)-(3.85), podemos perceber que as diferenças não são muito acentuadas. Vamos usar novamente o modelo de segunda ordem com constantes lineares $A_{y}=[0, \chi,-1]$ para o hamiltoniano efetivo (3.51) construído com as potências de $J_{y}$ e $A_{z}=[0, \chi,+1]$ para o hamiltoniano efetivo (3.51) construído com as potências de $J_{z}$. Vamos supor a presença de 10 estados vibracionais, $j=9$. Assim, os potenciais vibracionais da forma (3.80) podem ser escritos como

$$
\begin{aligned}
& V_{y}(q)=\frac{17}{72} q^{4}-\frac{17}{2} q^{2}-\frac{1}{2} q \sqrt{36-q^{2}} \chi \\
& V_{z}(q)=\frac{17}{72} q^{4}-\frac{17}{2} q^{2}+\frac{1}{2} q^{2} \chi
\end{aligned}
$$

onde $0 \leq q \leq 6$ quando há a presença de potências ímpares, devido à condição (2.44). Assim, quando $\chi=0$ estes dois potenciais reproduzem o mesmo potencial de Morse. No entanto, para valores nãonulos de $\chi$, eles apresentam diferenças sutis em relação ao potencial de Morse: (1) os pontos de mínimo deslocam-se ligeiramente; (2) a altura do potenciais também modificam-se ligeiramente e (3) estas duas modificações têm um comportamento anti-simétrico em relação aos valores positivos e negativos de $\chi$. Quando $\chi>0, V_{y}$ tem uma profundidade maior que a profundidade do potencial de Morse e um mínimo 
mais a direita que o mínimo de $V_{z}$, o qual tem uma profundidade menor que a profundidade do potencial de Morse e um mínimo ligeiramente à esquerda do mínimo do potencial de Morse. Esta situação está ilustrada na Figura 3.1 e é totalmente revertida quando $\chi<0$. Há diferenças também nos extremos, $q=0$ e $q=6$, mas estas diferenças dependem também das demais constantes lineares.

É interessante também fazermos uma comparação visual dos valores médios (3.83)-(3.85) após a transformação (3.75) ser aplicada $\operatorname{com} \theta=2 j$. As potências ímpares devem obedecer ao limite inferior $\beta \geq$ $-\ln 2$. As potências pares não precisam obedecer esta restrição. A Figura 3.2 mostra o comportamento dos oitos termos (3.83)-(3.85) para $j=9$. Três observações são importantes: (i) estes termos possuem intensidades em escalas diferentes; (ii) a largura também está ordenada, isto é, quanto maior a potência de $J_{y}$, menor a largura do respectivo valor médio (Figura 3.2(a)); (iii) os valores médios das potências ímpares de $J_{z}$ não são similares a um potencial de Morse. A primeira observação torna praticamente impossível a utilização da prescrição (3.80). A segunda observação pode ser útil para ajustar o alcance $\alpha$ em (3.75). $\mathrm{O}$ fato de haver outras potências, além de um e dois, cujos valores médios são similares a um potencial de Morse, sugere outras prescrições, similares a (3.81) e (3.82), para a construção de potenciais vibracionais. Uma outra observação também igualmente importante, a qual não está mostrada na Figura 3.2, é a presença de mais de um extremo nos valores médios das potências pares de $J_{y}$ maiores que dois. Para observarmos a presença destes extremos adicionais é necessário remover o limite inferior em $\beta$ e isto pode ser feito apenas com as potências pares. Por exemplo, os extremos do valor médio $\left\langle J_{y}^{4}\right\rangle$ dada em (3.85) são:

$$
\frac{d}{d q}\left\langle J_{y}^{4}\right\rangle=0 \Rightarrow\left\{\begin{array}{l}
q^{2}=2 j \Rightarrow \beta=0, \\
q^{2}=2 j\left\{1+\left[\frac{2 j}{2 j-3}+\frac{1}{(j-1)(2 j-3)}\right]^{\frac{1}{2}}\right\} \gtrsim 4 j \Rightarrow \beta \lesssim-\ln 2,
\end{array}\right.
$$

onde as aproximações valem para grandes valores de $j(j \gg 1)$. Considerando ainda o mesmo exemplo anterior com $j=9,-\left\langle J_{y}^{4}\right\rangle$ tem um mínimo em $\beta=0$, como mostrado na Figura 3.2, e um máximo em $\beta \approx-0,74$ (note que $-\ln 2 \approx-0,69$ ). Estes extremos adicionais poderão ser úteis na descrição de estados eletrônicos altamente excitados, onde os potenciais vibracionais podem apresentar mais de um extremo. Vale observar também que $\left\langle J_{z}^{4}\right\rangle$ não possui extremos adicionais.

\subsubsection{Fatores de Franck-Condon}

Quando ocorre uma transição eletrônica, por exemplo do estado eletrônico fundamental $X$ para o primeiro estado eletrônico excitado $A$, transições vibracionais entre estes estados também ocorrem. Devido à massa nuclear ser muito maior que a massa eletrônica, uma transição eletrônica deve ocorrer com a distância internuclear praticamente constante. Esta situação é conhecida como princípio de Franck-Condon. Do ponto de vista clássico, este princípio exige que a transição eletrônica seja feita de tal forma que a energia final coincida com um dos pontos de retorno do potencial eletrônico excitado. Assim, não haverá deslocamentos das distâncias nucleares enquanto a transição ocorre. Após a transição ser efetuada, então os núcleos passarão a vibrar com a nova energia. Em outras palavras, se os dois potenciais eletrônicos são colocados no mesmo gráfico, o princípio de Franck-Condon afirma que é necessário que uma reta vertical passando pelo ponto de mínimo do potencial menos excitado intercepte o potencial mais excitado em um de seus pontos de retorno. Do ponto de vista da Mecânica Quântica, o princípio de Franck-Condon exige que haja uma superposição não-nula entre a função de onda $|\varepsilon v\rangle$ do estado vibracional $|v\rangle$ no estado eletrônico menos excitado $|\varepsilon\rangle$ e a função de onda $\left|\varepsilon^{\prime} v^{\prime}\right\rangle$ do estado vibracional $\left|v^{\prime}\right\rangle$ no estado eletrônico mais excitado $\left|\varepsilon^{\prime}\right\rangle$. Quantitativamente, o princípio de Franck-Condon modela a forma das intensidades das transições eletrônicas de origem dipolar, $\mu=\mu_{e}+\mu_{n}$, onde $\mu_{e}$ e $\mu_{n}$ são as partes eletrônica e nuclear, respectivamente, do momento de dipolo $\mu$. Então, usando a aproximação de Born-Oppenheimer, $|\varepsilon v\rangle \approx|v\rangle|\varepsilon\rangle$, teremos

$$
\left\langle\varepsilon^{\prime} v^{\prime}|\mu| \varepsilon v\right\rangle=\left\langle v^{\prime} \mid v\right\rangle\left\langle\varepsilon^{\prime}\left|\mu_{e}\right| \varepsilon\right\rangle+\left\langle v^{\prime}\left|\mu_{n}\right| v\right\rangle\left\langle\varepsilon^{\prime} \mid \varepsilon\right\rangle=\left\langle v^{\prime} \mid v\right\rangle\left\langle\varepsilon^{\prime}\left|\mu_{e}\right| \varepsilon\right\rangle,
$$

onde usamos o fato dos estados eletrônicos serem ortogonais, $\left\langle\varepsilon^{\prime} \mid \varepsilon\right\rangle=0$. Desta forma, a intensidade da transição $|\varepsilon v\rangle \rightarrow\left|\varepsilon^{\prime} v^{\prime}\right\rangle$ é proporcional aos fatores de Franck-Condon

$$
F C\left(v^{\prime}, v\right)=\left|\left\langle v^{\prime} \mid v\right\rangle\right|^{2} .
$$

Quando os estados vibracionais estão normalizados, então os fatores de Franck-Condon satisfazem

$$
\sum_{v^{\prime}} F C\left(v^{\prime}, v\right)=\sum_{v^{\prime}}\left\langle v \mid v^{\prime}\right\rangle\left\langle v^{\prime} \mid v\right\rangle=\langle v \mid v\rangle=1 .
$$


Para o modelo algébrico, há duas alternativas para o cálculo dos fatores de Franck-Condon: (i) usando os autoestados da teoria de representação das álgebras envolvidas; (ii) usando funções de onda associadas aos autoestados da teoria de representação. Neste trabalho, usaremos apenas a segunda alternativa, por ser mais direta. Assim, usaremos as funções de onda radiais (3.24) encontradas na Seção 3.1.2 (Veja também o Apêndice B),

$$
R_{j m}(z)=C_{j m} \mathrm{e}^{-\frac{z}{2}} z^{m} L_{j-m}^{2 m}(z), \quad z=(2 j+1) \mathrm{e}^{-\beta}, \quad \beta=\alpha\left(r-r_{0}\right),
$$

onde fizemos $m=m_{y}, L_{n}^{\alpha}$ são as funções de Laguerre e $C_{j m}$ são as constantes de normalização

$$
C_{j m}=\sqrt{2 m \alpha}\left[\frac{(j-m) !}{(j+m) !}\right]^{\frac{1}{2}} .
$$

Assim, $R_{j m}$ são autofunções de $J_{y}$ na realização (3.29) (Veja também o Apêndice B), e estão devidamente normalizadas,

$$
\int_{0}^{\infty} \frac{d z}{\alpha z} R_{j m_{y}}(z) R_{j m_{y}^{\prime}}(z)=\delta_{m, m^{\prime}}
$$

Desta forma, os fatores de Franck-Condon no modelo algébrico podem ser calculados explicitamente por

$$
F C\left(j^{\prime} v^{\prime}, j v\right)=\left|\int_{0}^{\infty} \frac{d z}{\alpha z} R_{j m}(z) R_{j^{\prime} m^{\prime}}\left(z^{\prime}\right)\right|^{2}
$$

onde

$$
z^{\prime}=\left(2 j^{\prime}+1\right) \mathrm{e}^{-\alpha^{\prime}\left(r-r_{0}^{\prime}\right)}=z^{\gamma} \frac{\left(2 j^{\prime}+1\right)}{(2 j+1)^{\gamma}} \mathrm{e}^{\tau}, \quad \gamma:=\frac{\alpha^{\prime}}{\alpha}, \quad \tau:=\alpha^{\prime}\left(r_{0}^{\prime}-r_{0}\right) .
$$

Algumas observações importantes: (1) Note que o estado $m=0(v=j)$ tem uma função de onda nula em (3.91) devido à constante de normalização (3.92). Isto significa que o último estado $v=j$ nunca estará presente. (2) É necessário conhecermos os alcances $\alpha$ e $\alpha^{\prime}$ para calcularmos os fatores de Franck-Condon. Isto pode ser feito de duas maneiras. Eles podem ser determinados por um processo de otimização envolvendo os potenciais vibracionais algébricos e experimentais, ou eles podem ser determinados por um processo de otimização envolvendo diretamente os fatores de Franck-Condon (3.94) e aqueles determinados por outros métodos acurados. (3) Em geral, o uso das funções de Morse (3.91) é evitado em muitos outros trabalhos devido às instabilidades numéricas inerentes a elas. Estas instabilidades foram eliminadas no presente trabalho simplesmente usando uma forma recursiva para o cálculo das funções de Legendre. O uso da variável de Morse $z$ também é importante neste sentido.

\subsection{O método algébrico para moléculas poliatômicas}

A extensão do modelo descrito nas seções anteriores para o caso poli-atômico, restrito a sistemas moleculares planares, é feita, no que diz respeito ao procedimento para a confecção do hamiltoniano algébrico efetivo, associando um exemplar da álgebra su(2) a cada modo normal de vibração. Assim, necessitaremos de três exemplares da álgebra su(2) para moléculas tri-atômicas, duas delas serão associadas aos stretchings e a outra ao bending. No caso de moléculas tetra-atômicas, precisaremos de seis exemplares da álgebra $\mathrm{su}(2)$.

Vale destacar que o método algébrico unidimensional aplicado à sistemas moleculares maiores nos permite incluir a simetria pontual desses sistemas $\left(C_{2 v}\right.$ para os sistemas que estamos analisando, $\mathrm{H}_{2} \mathrm{O}$, $\mathrm{CO}_{2}$ e $\mathrm{H}_{2} \mathrm{CO}$ ) através dos operadores de Majorana, isto é, operadores construídos com elementos de exemplares diferentes das diversas álgebras su(2). Isto significa que as funções de onda vibracionais terão as devidas simetrias discretas já embutidas, sem a necessidade de projeções.

As dificuldades que deverão ser enfrentadas são várias: (1) falta de dados experimentais (principalmente envolvendo estados eletrônicos excitados, probabilidades de transições, etc.); (2) dificuldade em manusear as funções de onda num espaço de coordenadas devido à presença de bendings, os quais requerem que o operador $J_{z}$ seja diagonal, ao invés de $J_{y} ;(3)$ diagonalização dos operadores de Majorana e cálculo de seus valores médios via estados coerentes; (4) reconstrução dos potenciais vibracionais contendo as devidas interações não-lineares que poderão ocorrer entre os modos normais de vibração. 


\begin{tabular}{|c|c|c|l|}
\hline \hline Algébrico & MQ & Matsumoto & \\
\hline$\mu$ & $\mu$ & $m$ & massa reduzida \\
\hline$r_{0}$ & $r_{0}$ & $r_{e}$ & posição de equilíbrio \\
\hline$\alpha$ & $\alpha$ & $\alpha$ & alcance \\
\hline$D_{e}$ & $D_{e}$ & $D$ & profundidade \\
\hline$\frac{1}{2}(N+1)$ & $\lambda$ & $a$ & \\
\hline$m_{y}$ & $\frac{1}{2} \xi$ & $b$ & \\
\hline$v$ & $v$ & $n$ & \\
\hline \hline
\end{tabular}

Tabela 3.1: Relações entre as diversas constantes usadas na descrição do potencial de Morse, algebricamente (primeira coluna) e via equação de Schrödinger (segunda coluna). A terceira coluna mostra as mesmas constantes usadas por Matsumoto [79].

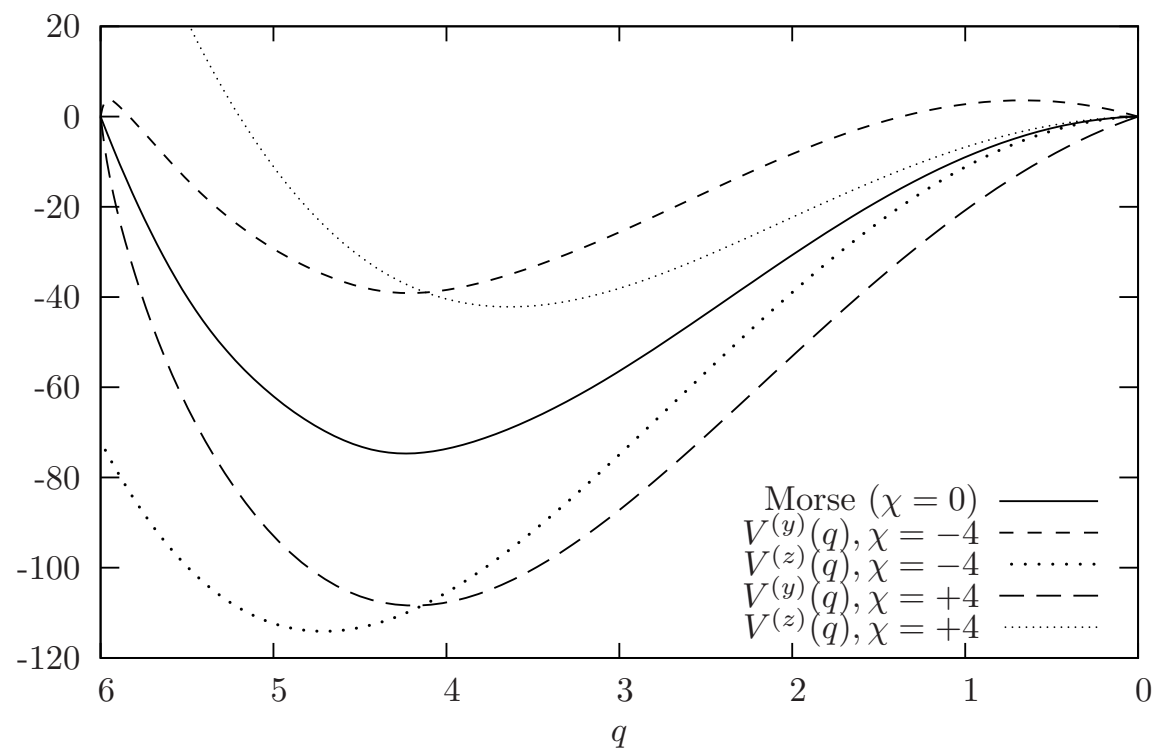

Figura 3.1: Situação fictícia para ilustrar algumas pequenas diferenças entre os potenciais obtidos através de hamiltonianos efetivos escritos em termos de $J_{y}$ e $J_{z}$. Os potenciais $V_{y}(q)$ e $V_{z}(q)$ estão dados em (3.86) e coincidem com o potencial de Morse quando $\chi=0$. 


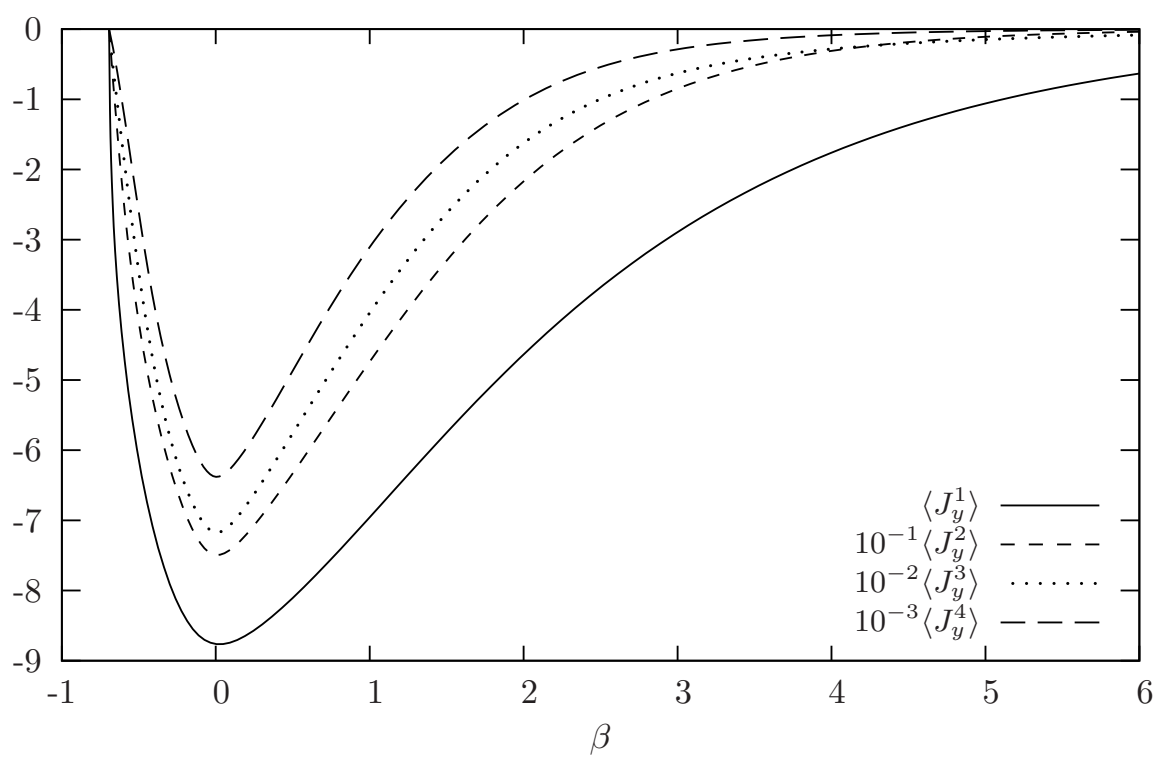

(a) Valores médios de $J_{y}$.

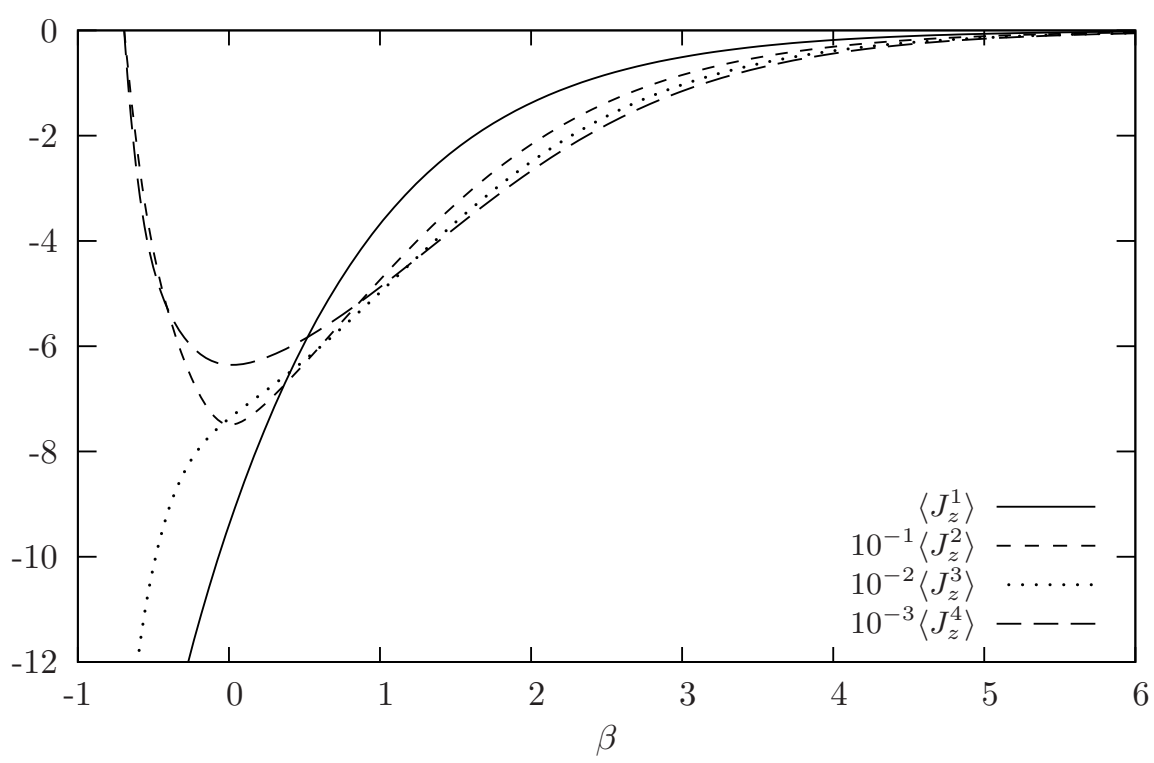

(b) Valores médios de $J_{z}$.

Figura 3.2: Valores médios das quatro primeiras potências de $J_{y}$ e $J_{z}$ para $j=9$, dadas em (3.83)-(3.85). A variável $\beta$ foi limitada inferiormente em $-\ln 2$. Note que a escala das amplitudes e o comportamento do valor médio das potências ímpares de $J_{z}$ são muito diferentes de um potencial de Morse. 


\section{Capítulo 4}

\section{Resultados e Discussões}

Apresentaremos neste capítulo, várias análises algébricas das energias vibracionais dos dois primeiros estados eletrônicos das moléculas diatômicas $\mathrm{LiH}$ (e seus principais isotopômeros), $\mathrm{NaH}, \mathrm{KH}, \mathrm{RbH}, \mathrm{CsH}$, $\mathrm{Cs}_{2}$ e $\mathrm{Li}_{2}$. Com exceção das duas moléculas diatômicas homonucleares, todas as constantes moleculares, energias vibracionais observadas e potenciais calculados foram retiradas de duas compilações feitas por Stwalley e Zemke e colaboradores [37, 38]. Evitaremos assim, por comodidade, citar os trabalhos originais referentes aos dados experimentais sobre as moléculas heteronucleares que usaremos nesta dissertação.

\section{$4.1 \quad \mathrm{LiH}$}

A situação experimental atual para os dois primeiros estados eletrônicos $\left(A-X^{1} \Sigma^{+}\right)$da molécula diatômica LiH e seus principais isotopômeros [38] está mostrada na Tabela 4.1. Podemos notar nesta tabela que nem todos os níveis vibracionais foram observados, como indicado pelos percentuais na terceira coluna. Por exemplo, apenas 10 níveis vibracionais foram observados para a molécula ${ }^{6} \mathrm{LiD}$. Isto significa que a energia vibracional mais alta que foi observada corresponde a $40 \%$ da profundidade $D_{e}$ (quinta coluna) da curva de potencial deste isotopômero. A Figura 4.1 mostra os potenciais dos dois primeiros estados eletrônicos para o isotopômero mais abundante ${ }^{7} \mathrm{LiH}$, reproduzidos aqui a partir dos dados apresentados na compilação [38]. Como indicado na Tabela 4.1, os demais potenciais para os outros isotopômeros são muito semelhantes àqueles mostrados na Figura 4.1. Nós excluímos o terceiro estado eletrônico $B^{1} \Pi$, também discutido na compilação [38], por possuir apenas quatro estados vibracionais. Outros dois estados mais excitados $\left(D-C^{1} \Sigma^{+}\right)$foram observados recentemente [86, 87, 88]. Há também resultados (mais cálculos do que observações experimentais) para algumas probabilidades de transição [89, 90], incluindo um análise dos fatores de Franck-Condon para os dois primeiros estados eletrônicos.

Nas sub-seções seguintes, iremos discutir em detalhes os resultados obtidos com dois modelos algébricos. Em um deles, iremos utilizar uma hamiltoniana algébrica efetiva contendo todas as potências de $J_{y}$, como em (3.51). Veremos que as energias vibracionais deste modelo serão similares às energias vibracionais provenientes da expansão de Dunham. No outro modelo, iremos usar apenas as potências pares de $J_{y}$, como tem sido feito tradicionalmente [25, 24]. Estas análises algébricas estão organizadas por estados eletrônicos e isotopômeros.

\subsubsection{Estado eletrônico fundamental $X^{1} \Sigma^{+}$}

Podemos ver na Figura 4.1 que o estado eletrônico fundamental tem um comportamento muito parecido com um potencial de Morse, isto é, tem um único mínimo, tem uma largura estreita e um alcance pequeno. Vejamos a seguir como estas características podem ser reproduzidas com modelos algébricos clássicos $(q=1)$. 


\section{Modelo algébrico clássico $(q=1)$ com todas as potências em $J_{y}$}

Vamos iniciar usando $n=9$ e $q=1$ (modelo clássico, sem deformação) em (3.51) para podermos comparar diretamente os valores dos nossos parâmetros lineares, após a mudança $v \rightarrow v+1 / 2$ em (3.55), com as constantes de Dunham em (3.10) que são recomendadas na literatura [38, Tabela 5]. Assim, devemos otimizar as dez constantes lineares $A_{i}$ presentes na expressão (3.55). Esta otimização linear é feita usando o desvio médio (3.57). A Tabela 4.2 mostra o resultado desta otimização. Este modelo apresenta somente uma constante arbitrária não-linear: $j$, a etiqueta das representações irredutíveis da álgebra su(2). Como observado no final da Seção 3.3.1, o desvio médio para este modelo não depende do número quântico $j$. Desta forma, temos a liberdade de escolher o melhor valor de $j$, levando em consideração os seguintes fatores. (1) $j+1$ deve ser o número de estados previstos pelos métodos ab initio. Não podemos esquecer que as funções de onda normalizadas (3.24) têm uma amplitude nula para o "último" estado $v=j$, devido à normalização ser diretamente proporcional a $m=j-v$. Assim, somos obrigados a adicionar uma unidade ao melhor valor de $j$. (2) A energia vibracional (3.55) do "último" estado, $E_{V}(j)=A_{0}$, deve estar próxima da energia de dissociação medida a partir do fundo do potencial, isto é, próxima da profundidade $D_{e}$ do potencial. Como $A_{0}$ é um polinômio em $j$, pudemos observar que, em geral, o primeiro máximo nas curvas $A_{0}(j)$ estão sempre muito próximos de $D_{e}$. Também observamos que os valores de $j$ onde ocorrem tais máximos, praticamente coincidem com o número de estados previstos em outros modelos. Estas duas observações estão ilustradas na Figura 4.2. Podemos ver claramente na Figura 4.2 que os máximos de $A_{0}$ ocorrem em $j=24$ para os dois primeiros isotopômeros $\left({ }^{7} \mathrm{LiH}\right.$ e $\left.{ }^{6} \mathrm{LiH}\right), j=31$ para ${ }^{7} \mathrm{LiD}$ e em $j=33$ para ${ }^{6} \mathrm{LiD}$. Estes valores de $j$ coincidem com as previsões obtidas extrapolando-se os valores apresentados na Tabela 4.1. Um terceiro fator, relacionado com a energia de dissociação $D_{0}=D_{e}-E_{V}(0)$, será discutido no final desta seção.

A Tabela 4.2 contém todos os dez parâmetros lineares (seis parâmetros lineares para o isotopômero $\left.{ }^{6} \mathrm{LiD}\right)$ do nosso modelo que serão usados para a construção dos potenciais vibracionais algébricos. O critério usado para determinarmos o melhor valor de $j$ é o mesmo apresentado no primeiro parágrafo e ilustrado na Figura 4.2. O único erro contido nos valores dos parâmetros algébricos apresentados na Tabela 4.2 é proveniente do arredondamento no último dígito, pois todo o processo de otimização foi feito inteiramente por computação simbólica (ou algébrica) onde trabalhamos com números racionais até a última etapa (3.61) da otimização. A partir destes valores numericamente exatos, nós calculamos todos os valores reais com uma precisão mínima de 20 dígitos. Este procedimento nos permite visualizar o comportamento do valor de cada parâmetro $A_{i}$, bem como o desvio médio e o melhor valor de $j$, em função do grau $n$ do polinômio (3.55). Este comportamento está mostrado nas Figuras 4.3, 4.4 e 4.5. O desvio médio mostrado na Figura 4.3 não depende de $j$ nesse modelo. Note que a convergência do desvio médio é obtida rapidamente. Os valores finais destes desvios médios estão mostrados na Tabela 4.2. A Figura 4.4 mostra o comportamento dos melhores valores de $j$, escolhidos no intervalo $j \pm 5$ de forma a obtermos o valor de $A_{0}$ mais próximo de $D_{e}$. Note na Figura 4.4 que, novamente, podemos perceber a similaridade forte entre as moléculas diferindo apenas pelos isótopos do lítio, ou, equivalentemente, diferindo apenas pelas massas reduzidas (veja a Tabela 4.1). Os valores da constante independente $A_{0}$, calculados a partir dos melhores valores de $j$, estão mostrados na Figura 4.5. Note a convergência do parâmetro $A_{0}$ para os três primeiros isotopômeros. O último isotopômero apresenta um comportamento ligeiramente diferente porque dispomos de poucas energias vibracionais observadas.

A Tabela 4.3 mostra uma comparação entre as duas principais constantes de Dunham, $Y_{10}$ e $Y_{20}$, calculadas aqui e aquelas apresentadas na Tabela 5 da Ref. [38]. Mostramos também na mesma tabela a correção $Y_{00}$, a qual não é independente no modelo de Dunham, mas é independente no nosso modelo algébrico. Como as demais constantes de Dunham apresentadas na Ref. [38, Tabela 5] apresentam com maior intensidade o efeito de contribuições adiabáticas, isto é, elas foram determinadas após a inclusão de correções à aproximação de Born-Oppenheimer ou, equivalentemente, determinadas por uma teoria de perturbação de segunda ordem, elas podem diferir significativamente das constantes calculadas aqui. Devemos observar também que, devido aos poucos dados experimentais disponíveis para o estado eletrônico fundamental do isotopômero ${ }^{6} \mathrm{LiD}$ (apenas 10 estados em um total de quase 30 esperados), optamos por usar apenas 6 constantes lineares, correspondendo a $n=5 \mathrm{em}$ (3.51), para podermos chegar o mais próximo possível da altura do potencial com o valor de $j$ em torno de 30 (veja a Figura 4.2). Podemos perceber então que este modelo algébrico é muito similar ao modelo de Dunham. Note também a similaridade forte entre os valores destas constantes para os isotopômeros do lítio. Como as constantes de Dunham $Y_{10}$ e $Y_{20}$ calculadas algebricamente estão muito próximas dos valores recomendados, elas satisfazem a relação (3.11). A Tabela 4.4 mostra as razões $r_{i 0}$ definidas em (3.11) para todas as 
combinações isotópicas. As demais constantes calculadas algebricamente, assim como as constantes de Dunham calculadas com correções adiabáticas, não satisfazem as relações (3.11). Isto significa que nossas constantes também refletem algum tipo de correção na aproximação de Born-Oppenheimer.

Uma vez tendo obtidos os melhores parâmetros algébricos, neste caso são os parâmetros mostrados na Tabela 4.2, podemos realizar outros testes, envolvendo as energias vibracionais diretamente, para checarmos a consistência das otimizações. Por exemplo, as diferenças sucessivas (Birge-Sponer) definidas em (3.68) constitui um excelente teste para qualquer processo de otimização. Primeiro, calculamos as diferenças sucessivas até ordem três para as energias observadas, as quais são muito regulares, e para as energias calculadas algebricamente. Estas diferenças sucessivas estão mostradas nas Figuras 4.6-4.8 para o isotopômero ${ }^{6} \mathrm{LiD}$, nosso pior resultado entre os quatro isotopômeros. Podemos ver nestas figuras que há uma previsão de 30 estados, aproximadamente. Este resultado está de acordo com outras previsões usando métodos ab initio. Como há mais energias observadas para os demais isotopômeros, as respectivas diferenças sucessivas, observadas e calculadas algebricamente, são muito similares. Isto pode ser melhor evidenciado, normalizando os valores absolutos destas diferenças sucessivas, as quais estão mostradas nas Figuras 4.9-4.12. Note que a diferença sucessiva de terceira ordem $\delta^{3}$ calculada para o isotopômero ${ }^{6} \mathrm{LiD}$ (Figura 4.12) é a única que não coincide "exatamente" com os valores observados, mas apresenta um comportamento qualitativo muito bom. Esta discrepância ocorre devido ã pouca quantidade de energias vibracionais observadas até o momento. Tendo poucas energias vibracionais observadas, somos obrigados a usar uma quantidade menor de parâmetros lineares $(n=5$, neste caso) no hamiltoniano algébrico. Podemos ver na Figura 4.6 que o modelo algébrico contém anarmonicidades, evidenciadas pelo desvio da linha cheia em relação a uma reta. Em geral, podemos concluir que as energias calculadas pelo método algébrico possuem um poder de previsão para as energias ainda não observadas.

Nesse modelo algébrico, os potenciais vibracionais são dados pela expressão (3.81). Como comentado no final da Seção 3.3.2, além das constantes físicas $R_{e}$ (posição de equilíbrio) e $\alpha$ (alcance), há a possibilidade de realizarmos ajustes na profundidade $V_{1}(r \rightarrow \infty)-V_{1}\left(R_{e}\right)$ do potencial algébrico $V_{1}(r)$ através da função $\theta_{1}(j)$ introduzida em (3.81). Para fixar a nomenclatura, denotaremos a profundidade do potencial algébrico por $D_{0}^{A}$ quando não introduzirmos qualquer ajuste, ou seja, quando usarmos $\theta_{1}(j)=j$ em (3.81). Quando fizermos o primeiro ajuste $j \rightarrow j+1 / 2$, isto é, $\theta_{1}(j)=j+1 / 2$, iremos utilizar $D_{e}^{A}$ para denotar a profundidade do potencial algébrico. Iremos utilizar também $D_{e}^{A}$ para denotar a profundidade do potencial algébrico mesmo quando fizermos um ajuste fino: $\theta_{1}(j)=j+1 / 2+\delta j$, onde $\delta j$ será uma quantidade real pequena. Neste trabalho, para efeito de comparação, usaremos os potenciais obtidos através do método RKR [91, 92], ou alguma de suas variantes, ou o método IPA [93, 94], por serem muito precisos, confiáveis e amplamente conhecidos. A Figura 4.13 mostra o potencial IPA e o potencial determinado algebricamente como prescrito em (3.81), usando os parâmetros dados na Tabela 4.2. Em todos os casos neste trabalho, iremos usar os valores recomendados para as posições de equilíbrio apresentados na Tabela 4.1. Mostramos na Figura 4.13 duas curvas de potencial: uma calculada com o alcance $\alpha=1.21 / A$ e a outra com $\alpha=1.41 / A$. Podemos notar então que a largura do potencial vibracional algébrico é sempre ligeiramente diferente da largura do potencial IPA. Nesta figura, escolhemos o valor $\theta_{1}(j)=j+1 / 2+\delta j$, onde $\delta j=0.05358$, para a função ajuste em (3.81). A Tabela 4.5 mostra alguns valores interessantes para esta função ajuste. Podemos ver que no caso onde não há qualquer tipo de ajuste, primeira linha, a profundidade do potencial algébrico está muito próxima da energia de dissociação observada $D_{0}$ para cada isotopômero. Quando fazemos o primeiro ajuste, a troca $j \rightarrow j+1 / 2$ na quinta linha, a situação muda significativamente, pois agora a profundidade do potencial algébrico está mais próxima da profundidade $D_{e}$ do potencial IPA. Note também que as diferenças $D_{0}-D_{0}^{A}$ e $D_{e}-D_{e}^{A}$ nestes dois casos são praticamente as mesmas. Um ajuste fino é feito na sétima linha, escolhendo os valores de $\delta j$ para nos aproximarmos satisfatoriamente de $D_{e}$. As curvas de potencial para os demais isotopômeros têm o mesmo comportamento.

Vale mencionar que escolhemos $j=30$ como sendo o melhor valor no processo de otimização para o isotopômero ${ }^{6} \mathrm{LiD}$, seguindo os critérios apresentados no início desta seção. Esta escolha é re-confirmada comparando as diferenças $D_{0}-D_{0}^{A}$ para alguns valores de $j$. Por exemplo, quando $j=29, D_{0}-D_{0}^{A}=$ $632.1 \mathrm{~cm}^{-1}$; quando $j=31, D_{0}-D_{0}^{A}=653.8 \mathrm{~cm}^{-1}$. Então, o melhor valor é $j=30$, pois a diferença $D_{0}-D_{0}^{A}=601.8 \mathrm{~cm}^{-1}$ é a menor possível. Este é o outro fator, mencionado no início desta seção, que usamos para escolher os melhores valores de $j$ neste trabalho. 


\section{Modelo algébrico clássico $(q=1)$ com as potências pares em $J_{y}$}

Realizamos vários testes envolvendo todos os sistemas diatômicos em estudo neste trabalho e não encontramos qualquer aspecto diferente que indicasse a necessidade de usar este modelo. Considerando que o desvio médio neste modelo também depende de $j$, o número quântico da representação irredutível da álgebra su(2), ele é menos atrativo do ponto de vista computacional. Desta forma, preferimos evitar uma discussão deste modelo neste momento.

\subsubsection{Primeiro estado eletrônico excitado $A^{1} \Sigma^{+}$}

Como pode ser visto na Figura 4.1, o primeiro estado eletrônico excitado $A^{1} \Sigma^{+}$também tem um único mínimo, mas uma largura maior que o estado fundamental. Embora este potencial ainda nos lembre um potencial de Morse, veremos a seguir, que a descrição algébrico deste estado eletrônico será diferente da descrição algébrica do estado eletrônico fundamental.

\section{Modelo algébrico clássico $(q=1)$ com todas as potências em $J_{y}$}

De forma análoga ao caso anterior, usamos todas as potências em (3.51) e $q=1$. Assim, para cada isotopômero, otimizamos as constantes linearmente independentes presentes na energia vibracional (3.55) para diversos valores de $n$. Apesar do desvio médio, para todos os isotopômeros, estar abaixo de $1 / 100 \mathrm{~cm}^{-1}$ quando $n=9$, as energias de dissociação dos potenciais vibracionais algébricos, bem como as profundidades, ficaram muito abaixo dos valores previstos diretamente das observações experimentais. Desta forma, preferimos prosseguir usando o modelo algébrico construído usando somente as potências pares de $J_{y}$, como descrito a seguir.

\section{Modelo algébrico clássico $(q=1)$ com as potências pares em $J_{y}$}

Neste modelo, usamos apenas as potências pares em (3.52) e $q=1$ (caso clássico, sem deformação). Desta forma pudemos reproduzir adequadamente as principais características do espectro vibracional de cada isotopômero. Iniciamos determinando os parâmetros lineares do modelo em função do parâmetro não-linear $j$. Usamos os seguintes critérios para escolhermos os melhores valores de $j$ : (i) o valor de $j$ que resulta em um menor desvio médio e em um valor para a constante independente $B_{0}$ próximo da energia de dissociação esperada $D_{0}$; (ii) o valor $j$ mais próximo do número de níveis vibracionais observados; (iii) o valor de $j$ que dê uma altura para o potencial algébrico (3.82) próxima da altura do potencial IPA. Em geral, observamos que os dois primeiros critérios são suficientes. O desvio médio sempre apresenta um mínimo local para um determinado valor de $j$ em torno do número de energias vibracionais observadas. Os melhores valores dos parâmetros algébricos estão mostrados na Tabela 4.6. Também calculamos as três primeiras constantes de Dunham, as quais estão mostradas na Tabela 4.7, a partir dos parâmetros algébricos da Tabela 4.6. Podemos observar uma boa concordância com as constantes de Dunham (com correções adiabáticas) recomendadas [38, Tabela 6]. Estas três constantes de Dunham calculadas algebricamente também satisfazem a relação (3.11) (Veja a Tabela 4.4). Apesar das demais constantes de Dunham do modelo algébrico não satisfazerem exatamente a relação (3.11), elas a satisfazem de forma melhor que as mesmas constantes calculadas para o estado eletrônico fundamental. Note a semelhança grande entre os dois isotopômeros ${ }^{7} \mathrm{LiH}$ e ${ }^{6} \mathrm{LiH}$, quando comparamos os valores dos respectivos parâmetros na Tabela 4.6. A mesma observação vale para os dois isotopômeros ${ }^{7} \mathrm{LiD}$ e ${ }^{6} \mathrm{LiD}$. Diferentemente, no estado eletrônico fundamental, esta semelhança aparece entre os isotopômeros ${ }^{7} \mathrm{LiH}-$ ${ }^{7} \mathrm{LiD} \mathrm{e}{ }^{6} \mathrm{LiH}-{ }^{6} \mathrm{LiD}$, como pode ser visto na Tabela 4.2 .

Conforme indicado na Tabela 4.6, os desvios médios obtidos com nove $(n=8)$ parâmetros lineares são muito pequenos. Há nenhuma melhora sensível para $n>8$. A Figura 4.15 (Birge-Sponer) ilustra o comportamento das diferenças sucessivas de primeira ordem $\Delta^{1}$, definidas em (3.68), para os isotopômeros da molécula LiH. Note que temos um poder de previsão razoável, pois apenas metade (aproximadamente) das energias vibracionais foram observadas até o momento para cada isotopômero. Esta figura nos mostra também que o espectro vibracional para este primeiro estado eletrônico excitado é muito mais anarmônico que o estado eletrônico fundamental (veja a Figura 4.6. Quando não é possível fazer previsões com o modelo algébrico, não é possível fazer as extrapolações Birge-Sponer mostradas na Figura 4.15. Os valores de $j$ apresentados no Tabela 4.6 correspondem ao número máximo de estados vibracionais previstos 
algebricamente e estão de acordo com outras previsões teóricas [89] e com as extrapolações Birge-Sponer mostradas na Figura 4.15. A Figura 4.16 ilustra, usando o isotopômero ${ }^{7} \mathrm{LiH}$, o comportamento típico das diferenças sucessivas até terceira ordem.

Nesse modelo, os potenciais vibracionais algébricos são dados pela expressão (3.82). Analogamente ao que fizemos para o estado eletrônico fundamental, denotaremos por $D_{0}^{A}$ a altura do potencial algébrico quando não introduzirmos qualquer ajuste, ou seja, $\theta_{2}(j)=2 j(2 j-1) / 4$, como indicado em (3.82). Quando fizermos o primeiro ajuste $\theta_{2}(j)=(j+1)^{2}$, iremos utilizar $D_{e}^{A}$ para denotar a profundidade do potencial algébrico. Inclusive, iremos utilizar $D_{e}^{A}$ para denotar a profundidade do potencial algébrico mesmo quando fizermos um ajuste fino: $\theta_{1}(j)=(j+1+\delta j)^{2}$, onde $\delta j$ será uma quantidade real pequena. A Figura 4.14 mostra o potencial IPA e o potencial determinado algebricamente como prescrito em (3.82), usando os parâmetros dados nas Tabelas 4.6 e 4.1, para dois valores do alcance $\alpha$. Podemos notar novamente que a largura do potencial vibracional algébrico é diferente da largura do potencial IPA, desta vez de forma mais acentuada. A Tabela 4.8 mostra alguns valores interessantes da função $\theta_{2}$ para todos os isotopômeros LiH. Observamos aqui o mesmo comportamento verificado para o estado eletrônico fundamental: quando não há qualquer tipo de ajuste, primeira linha da Tabela 4.8, a profundidade do potencial algébrico está muito próxima da energia de dissociação observada $D_{0}$ para cada isotopômero. Quando fazemos o primeiro ajuste na quinta linha, a situação muda significativamente, pois agora a profundidade do potencial algébrico está mais próxima da profundidade $D_{e}$ do potencial IPA. Um ajuste fino é feito na sétima linha. As curvas de potencial para os demais isotopômeros têm o mesmo comportamento.

\subsubsection{Fatores de Frank-Condon entre os estados $A-X^{1} \Sigma^{+}$}

Calculamos os fatores de Franck-Condon (3.94) para as transições entre os estados vibracionais dos estados eletrônicos $X^{1} \Sigma^{+}$e $A^{1} \Sigma^{+}$do ${ }^{7} \mathrm{LiH}$. Usamos os valores das posições de equilíbrio dadas na Tabela 4.1. Os valores para os alcances foram obtidos através de uma otimização (método dos mínimos quadrados) comparando nossos resultados com os mesmos fatores de Franck-Condon obtidos por métodos ab initio [89]. Os valores do alcance $\alpha$ provenientes destas otimizações estão muito próximos dos valores indicados nas Figuras 4.13 e 4.14. Os valores de $j$ foram os mesmos obtidos nas otimizações cujos parâmetros estão mostrados nas Tabelas 4.2 e 4.6. As Figuras 4.17-4.18 mostram os fatores de FranckCondon (adimensionais e normalizados) para as quatro primeiras bandas. Em cada banda, a abscissa mostra os números quânticos para as energias vibracionais do estado eletrônico fundamental $X^{1} \Sigma^{+}$. Como estes fatores estão normalizados, então a soma de todos eles para cada banda deve ser a unidade. Isto é verificado para a maioria das primeiras bandas. As últimas bandas apresentam valores para a soma dos fatores de Franck-Condon que podem diferir apreciavelmente da unidade. Esta situação também é observada nos cálculos feitos por métodos ab initio [89]. Mostramos também nas Figuras 4.17-4.18 os mesmos fatores de Franck-Condon calculados por um outro método [89], uma vez que estes cálculos concordam bem com o comportamento geral das intensidades observadas. Os fatores de Franck-Condon para as demais bandas também concordam qualitativamente com a descrição qualitativa das intensidades reportadas em [89], mas diferem quantitativamente dos valores calculados em [89].

\subsection{Demais moléculas diatômicas alcalinas}

Fizemos um estudo detalhado dos dois primeiros estados eletrônicos $X^{1} \Sigma^{+}$e $A^{1} \Sigma^{+}$para os hidretos alcalinos $\mathrm{NaH}, \mathrm{KH}, \mathrm{RbH}$ e CsH, de forma idêntica aos estudos feitos para LiH. Em geral, o comportamento observado foi muito similar: (1) o estado eletrônico fundamental $X^{1} \Sigma^{+}$é descrito pela hamiltoniana algébrica clássica $(q=1)$ (3.51) (todas as potências) com um número de parâmetros lineares em torno de 10 e um desvio médio da ordem de $1 / 1000 \mathrm{~cm}^{-1} ;(2)$ o primeiro estado eletrônico excitado $A^{1} \Sigma^{+}$é descrito pelo hamiltoniana clássica (3.52) (potências pares); (3) em ambos os casos, precisamos de ajustes na profundidade do potencial; (4) em ambos os casos, o alcance do potencial algébrico é ligeiramente diferente do alcance do potencial RKR e IPA; (5) em ambos os casos, podemos fazer previsões sobre o número máximo de estados vibracionais, as quais estão de acordo com as previsões experimentais. 


\section{$4.3 \quad \mathrm{Li}_{2}$}

Realizamos também um estudo dos estados eletrônicos $X^{1} \Sigma_{g}^{+}$e $A^{1} \Sigma_{u}^{+}$da molécula $\mathrm{Li}_{2}$. Diferentemente dos demais casos, estes dois estados podem ser descritos muito bem pela mesma hamiltoniana (3.51) (todas as potências) com um número de parâmetros lineares em torno de 12 e um desvio médio em torno $1 / 100000 \mathrm{~cm}^{-1}$. Este desvio médio baixo é possível devido à grande quantidade de energias vibracionais observadas (mais de $98 \%$ da profundidade dos potenciais) e à grande precisão nos dados observados. Em ambos os estados eletrônicos, precisamos também realizar ajustes na profundidade dos potenciais, no entanto, podemos afirmar que este é o caso onde há uma melhor concordância entre os potenciais determinados algebricamente e os potenciais RKR (com correções de longo alcance).

\begin{tabular}{|c|c|c|c|c|l|l|}
\hline \hline Isotopômero & Estado & $N_{e}$ & $T_{e}\left(\mathrm{~cm}^{-1}\right)$ & $D_{e}\left(\mathrm{~cm}^{-1}\right)$ & $R_{e}(A)$ & $\mu$ \\
\hline${ }^{7} \mathrm{LiH}$ & $X^{1} \Sigma^{+}$ & $23(98 \%)$ & & $20287.7 \pm 0.3$ & 1.595584 & 0.88123816 \\
\hline${ }^{6} \mathrm{LiH}$ & $X^{1} \Sigma^{+}$ & $22(90 \%)$ & & $20287.7 \pm 0.4$ & 1.595584 & 0.86319752 \\
\hline${ }^{7} \mathrm{LiD}$ & $X^{1} \Sigma^{+}$ & $28(90 \%)$ & & $20293.5 \pm 0.3$ & 1.595304 & 1.56487045 \\
\hline${ }^{6} \mathrm{LiD}$ & $X^{1} \Sigma^{+}$ & $10(40 \%)$ & & $20293.5 \pm 0.4$ & 1.595304 & 1.50887159 \\
\hline${ }^{7} \mathrm{LiH}$ & $A^{1} \Sigma^{+}$ & $16(80 \%)$ & 26509.77 & $8681.6 \pm 0.3$ & 2.59628 & \\
\hline${ }^{6} \mathrm{LiH}$ & $A^{1} \Sigma^{+}$ & $17(80 \%)$ & 26509.68 & $8681.6 \pm 0.4$ & 2.59647 & \\
\hline${ }^{7} \mathrm{LiD}$ & $A^{1} \Sigma^{+}$ & $19(70 \%)$ & 26512.52 & $8684.7 \pm 0.3$ & 2.59690 & \\
\hline${ }^{6} \mathrm{LiD}$ & $A^{1} \Sigma^{+}$ & $19(70 \%)$ & 26512.44 & $8684.7 \pm 0.4$ & 2.59710 & \\
\hline \hline
\end{tabular}

Tabela 4.1: Constantes moleculares recomendadas [38] para a molécula LiH e seus principais isotopômeros. $X^{1} \Sigma^{+}$representa o estado eletrônico fundamental e $A^{1} \Sigma^{+}$o primeiro estado eletrônico excitado. $N_{e}$ representa o número de níveis vibracionais observados em cada estado eletrônico. Os percentuais na terceira coluna indicam a quantidade de níveis observados em relação ao total previsto. A quarta coluna mostra as energias eletrônicas $T_{e}$, medidas entre o mínimo do potencial eletrônico fundamental e o mínimo do potencial eletrônico excitado. A profundidade $D_{e}=V(r \rightarrow \infty)-V\left(R_{e}\right)$, onde $V(r)$ é o potencial eletrônico, é mostrada na quinta coluna. A sexta coluna mostra as posições de equilíbrio $R_{e}$, medidas em Angstrons. As massa reduzidas, medidas em unidades de massa atômica $\left(=M_{12} \mathrm{C} / 12\right)$, estão na última coluna. 


\begin{tabular}{|c|c|c|c|c|}
\hline$X^{1} \Sigma^{+}$ & ${ }^{7} \mathrm{LiH}$ & ${ }^{6} \mathrm{LiH}$ & ${ }^{7} \mathrm{LiD}$ & ${ }^{6} \mathrm{LiD}$ \\
\hline$A_{0}$ & $2.02130754 E+04$ & $2.01566611 E+04$ & $2.02006005 E+04$ & $1.96917063 E+04$ \\
\hline$A_{1}$ & $1.49718844 E+02$ & $2.13721740 E+02$ & $1.20121199 E+02$ & $9.72805664 E+00$ \\
\hline$A_{2}$ & $-9.58955888 E+01$ & $-1.07181462 E+02$ & $-6.01018964 E+01$ & $-4.05864773 E+01$ \\
\hline$A_{3}$ & $5.48444793 E+00$ & $6.95017932 E+00$ & $3.43601436 E+00$ & $1.16843124 E+00$ \\
\hline$A_{4}$ & $-1.83410568 E-01$ & $-3.36276887 E-01$ & $-1.71344543 E-01$ & $-2.30168998 E-02$ \\
\hline$A_{5}$ & $-2.23688372 E-03$ & $9.44147688 E-03$ & $6.55046705 E-03$ & $1.71794872 E-04$ \\
\hline$A_{6}$ & $5.00097830 E-04$ & $-1.07122196 E-04$ & $-1.91827419 E-04$ & \\
\hline$A_{7}$ & $-2.17203684 E-05$ & $-1.72266999 E-06$ & $3.98680068 E-06$ & \\
\hline$A_{8}$ & $4.41331157 E-07$ & $6.76219115 E-08$ & $-5.10543134 E-08$ & \\
\hline$A_{9}$ & $-3.61756730 E-09$ & $-6.07595425 E-10$ & $2.96333614 E-10$ & \\
\hline$j$ & 24 & 24 & 32 & 30 \\
\hline rms & 0.0106 & 0.0080 & 0.0018 & 0.0046 \\
\hline
\end{tabular}

Tabela 4.2: Parâmetros da hamiltoniana vibracional efetiva (3.51) (todas as potências) para o estado eletrônico fundamental $X^{1} \Sigma^{+}$dos isotopômeros da molécula LiH. Os parâmetros lineares $A_{i}$ foram determinadas algebricamente pelo método dos mínimos quadrados. A precisão em cada parâmetro é unicamente o erro de arredondamento no último dígito. Todos os parâmetros e o desvio médio estão em $\mathrm{cm}^{-1}$. Neste modelo, o desvio médio $\mathrm{rms}$ é independente do parâmetro não-linear (adimensional) $j$, a etiqueta $j$ da representação irredutível da álgebra su(2).

\begin{tabular}{|l|r|r|r|r|r|}
\hline \hline$X^{1} \Sigma^{+}$ & & ${ }^{7} \mathrm{LiH}$ & ${ }^{6} \mathrm{LiH}$ & ${ }^{7} \mathrm{LiD}$ & ${ }^{6} \mathrm{LiD}$ \\
\hline $\mathrm{SZ}$ & $Y_{00}$ & 1.06080 & 1.08299 & 0.52939 & 0.54903 \\
\hline $\mathrm{Alg}$. & $Y_{00}$ & 0.77172 & 0.89053 & 0.56634 & 0.57292 \\
\hline $\mathrm{SZ}$ & $Y_{10}$ & 1405.50936 & 1420.11754 & 1054.93684 & 1074.33199 \\
\hline $\mathrm{Alg}$. & $Y_{10}$ & 1405.86333 & 1420.15653 & 1054.66575 & 1074.12065 \\
\hline $\mathrm{SZ}$ & $Y_{20}$ & -23.17938 & -23.66382 & -13.05489 & -13.53939 \\
\hline $\mathrm{Alg}$. & $Y_{20}$ & -23.46457 & -23.66021 & -12.85165 & -13.40113 \\
\hline \hline
\end{tabular}

Tabela 4.3: Comparação entre as três primeiras constantes de Dunham recomendadas [38] (SZ) e calculadas algebricamente (Alg.), para o estado eletrônico fundamental dos isotopômeros da molécula LiH. Os parâmetros livres do modelo algébrico estão na Tabela 4.2. Todas as constantes $Y_{i j}$ estão em $\mathrm{cm}^{-1}$. 


\begin{tabular}{|c|c|c|c|c|r|r|r|}
\hline \hline & & $\mathrm{a}-\mathrm{b}$ & $\mathrm{a}-\mathrm{c}$ & $\mathrm{a}-\mathrm{d}$ & $\mathrm{b}-\mathrm{c}$ & $\mathrm{b}-\mathrm{d}$ & $\mathrm{c}-\mathrm{d}$ \\
\hline$X^{1} \Sigma^{+}$ & $r_{10}$ & 1.00023 & 1.00031 & 1.00025 & 1.00009 & 1.00003 & 0.99994 \\
$X^{1} \Sigma^{+}$ & $r_{20}$ & 1.01246 & 1.02818 & 1.02261 & 1.01553 & 1.01003 & 0.99459 \\
\hline$A^{1} \Sigma^{+}$ & $r_{10}$ & 1.00502 & 0.99198 & 0.99242 & 0.98703 & 0.98747 & 1.00045 \\
$A^{1} \Sigma^{+}$ & $r_{20}$ & 0.95913 & 1.02427 & 1.02325 & 1.06792 & 1.06685 & 0.99900 \\
\hline \hline
\end{tabular}

Tabela 4.4: Reescalonamento (3.11) das constantes de Dunham calculadas algebricamente (Tabelas 4.3 e 4.7) para os isotopômeros $\mathrm{a}={ }^{7} \mathrm{LiH}, \mathrm{b}={ }^{6} \mathrm{LiH}, \mathrm{c}={ }^{7} \mathrm{LiD}$ e $\mathrm{d}={ }^{6} \mathrm{LiD}$. As massas reduzidas estão apresentadas na Tabela 4.1. $X^{1} \Sigma^{+}$representa o estado eletrônico fundamental e $A^{1} \Sigma^{+}$o primeiro estado eletrônico excitado.

\begin{tabular}{|c|r|r|r|r|}
\hline \hline$X^{1} \Sigma^{+}$ & ${ }^{7} \mathrm{LiH}$ & ${ }^{6} \mathrm{LiH}$ & ${ }^{7} \mathrm{LiD}$ & ${ }^{6} \mathrm{LiD}$ \\
\hline$\theta_{1}=j$ & 24 & 24 & 32 & 30 \\
\hline$D_{0}-D_{0}^{A}$ & 74.5961 & 131.0143 & 92.8872 & 601.7821 \\
\hline$\%$ & 0.38 & 0.67 & 0.47 & 3.05 \\
\hline$\theta_{1}=j+1 / 2$ & $24+1 / 2$ & $24+1 / 2$ & $32+1 / 2$ & $30+1 / 2$ \\
\hline$D_{e}-D_{e}^{A}$ & 75.3964 & 131.9294 & 93.4658 & 602.3667 \\
\hline$\%$ & 0.37 & 0.65 & 0.46 & 2.97 \\
\hline$\theta_{1}=j+1 / 2+\delta j$ & 24.55358 & 24.59275 & 32.58852 & 31.05692 \\
\hline$D_{e}-D_{e}^{A}$ & 0.0028 & 0.0062 & 0.0061 & 0.0040 \\
\hline \hline
\end{tabular}

Tabela 4.5: Dois tipos de ajustes feitos na profundidade dos potenciais vibracionais algébricos (3.81) para o estado eletrônico fundamental $X^{1} \Sigma^{+}$dos isotopômeros da molécula LiH. $D_{0}^{A}$ representa a profundidade do potencial algébrico (3.81) quando $\theta_{1}=j . D_{e}^{A}$ representa a profundidade do potencial algébrico (3.81) quando $\theta_{1}=j+1 / 2$ ou $\theta_{1}=j+1 / 2+\delta j$ (correções). $D_{e}$ representa a altura do potencial RKR ou IPA, $D_{e}=V(r \rightarrow \infty)-V\left(R_{e}\right)$, onde $R_{e}$ é a posição de equilíbrio, e $D_{0}$ é a energia de dissociação, $D_{0}=D_{e}-E(0)$. Os percentuais na quarta e na sétima linhas são calculados em relação a $D_{0}$ e $D_{e}$, respectivamente. Todas as energias estão em $\mathrm{cm}^{-1}$. 


\begin{tabular}{|c|c|c|c|c|}
\hline$A^{1} \Sigma^{+}$ & ${ }^{7} \mathrm{LiH}$ & ${ }^{6} \mathrm{LiH}$ & ${ }^{7} \mathrm{LiD}$ & ${ }^{6} \mathrm{LiD}$ \\
\hline$B_{0}$ & $8.75841644 E+03$ & $8.60929836 E+03$ & $8.66543147 E+03$ & $8.59774971 E+03$ \\
\hline$B_{1}$ & $-2.50704025 E+01$ & $-2.75135005 E+01$ & $-1.44265979 E+01$ & $-1.51365252 E+01$ \\
\hline$B_{2}$ & $5.95526651 E-02$ & $7.64199362 E-02$ & $1.99517972 E-02$ & $2.19673357 E-02$ \\
\hline$B_{3}$ & $-1.75050067 E-04$ & $-2.54716703 E-04$ & $-3.39250026 E-05$ & $-3.89362855 E-05$ \\
\hline$B_{4}$ & $3.99475905 E-07$ & $6.45475816 E-07$ & $4.47727352 E-08$ & $5.36469558 E-08$ \\
\hline$B_{5}$ & $-6.25360910 E-10$ & $-1.10926086 E-09$ & $-4.05179572 E-11$ & $-5.08173552 E-11$ \\
\hline$B_{6}$ & $6.32950749 E-13$ & $1.22023488 E-12$ & $2.36701458 E-14$ & $3.11595454 E-14$ \\
\hline$B_{7}$ & $-3.71343736 E-16$ & $-7.72006676 E-16$ & $-7.99964054 E-18$ & $-1.10798280 E-17$ \\
\hline$B_{8}$ & $9.60486334 E-20$ & $2.13843992 E-19$ & $1.18944775 E-21$ & $1.73693220 E-21$ \\
\hline$j$ & 28 & 27 & 37 & 36 \\
\hline rms & 0.0011 & 0.0069 & 0.0028 & 0.0024 \\
\hline
\end{tabular}

Tabela 4.6: Parâmetros da hamiltoniana vibracional efetiva (3.52) (potências pares) para o primeiro estado eletrônico excitado $A^{1} \Sigma^{+}$dos isotopômeros da molécula LiH. Os parâmetros lineares $B_{i}$ foram determinadas algebricamente pelo método dos mínimos quadrados. A precisão em cada parâmetro é unicamente o erro de arredondamento no último dígito. Todos os parâmetros e o desvio médio estão em $\mathrm{cm}^{-1}$. Neste modelo, o desvio médio $\mathrm{rms}$ é dependente do parâmetro não-linear (adimensional) $j$, a etiqueta $j$ da representação irredutível da álgebra su(2).

\begin{tabular}{|c|r|r|r|r|r|}
\hline \hline${ }^{1} \Sigma^{+}$ & & ${ }^{7} \mathrm{LiH}$ & ${ }^{6} \mathrm{LiH}$ & ${ }^{7} \mathrm{LiD}$ & ${ }^{6} \mathrm{LiD}$ \\
\hline Alg. & $Y_{00}$ & 7.74191 & 8.19038 & 4.29094 & 4.45403 \\
\hline S.Z. & $Y_{00}$ & 7.31415 & 7.46699 & 3.94037 & 4.08900 \\
\hline Alg. & $Y_{10}$ & 232.62692 & 233.87187 & 175.98091 & 179.13631 \\
\hline S.Z. & $Y_{10}$ & 234.50825 & 236.93379 & 178.57167 & 181.84580 \\
\hline Alg. & $Y_{20}$ & 31.50739 & 33.53660 & 17.32251 & 17.98343 \\
\hline S.Z. & $Y_{20}$ & 28.81350 & 29.41569 & 15.3183 & 15.88681 \\
\hline \hline
\end{tabular}

Tabela 4.7: Comparação entre as três primeiras constantes de Dunham recomendadas [38] (SZ) e calculadas algebricamente (Alg.), para o primeiro estado eletrônico excitado $A^{1} \Sigma^{+}$dos isotopômeros da molécula LiH. Os parâmetros livres do modelo algébrico estão na Tabela 4.6. Todas as constantes $Y_{i j}$ estão em $\mathrm{cm}^{-1}$. A Tabela 4.4 mostra o reescalonamento destas constantes com as massas reduzidas. 


\begin{tabular}{|c|r|r|r|r|}
\hline \hline$A^{1} \Sigma^{+}$ & ${ }^{7} \mathrm{LiH}$ & ${ }^{6} \mathrm{LiH}$ & ${ }^{7} \mathrm{LiD}$ & ${ }^{6} \mathrm{LiD}$ \\
\hline$j$ & 28 & 27 & 37 & 36 \\
\hline$\theta_{2}=2 j(2 j-1) / 4$ & 770 & 715.5 & 1350.5 & 1278 \\
\hline$D_{0}-D_{0}^{A}$ & -10.0447 & 139.7766 & 67.6358 & 136.2768 \\
\hline$\%$ & -0.12 & 1.63 & 0.79 & 1.59 \\
\hline$\theta_{2}=(j+1)^{2}$ & 841 & 784 & 1444 & 1369 \\
\hline$D_{e}-D_{e}^{A}$ & -176.6937 & -27.09232 & -59.7717 & 6.6798 \\
\hline$\%$ & -2.04 & -0.31 & -0.69 & 0.08 \\
\hline$\theta_{2}=(j+1+\delta j)^{2}$ & -0.78639 & -0.13520 & -0.36430 & +0.04226 \\
\hline$D_{e}-D_{e}^{A}$ & 0.0002 & 0.0031 & 0.0040 & 0.0004 \\
\hline \hline
\end{tabular}

Tabela 4.8: Dois tipos de ajustes feitos na profundidade dos potenciais vibracionais algébricos (3.82) para o primeiro estado eletrônico excitado $A^{1} \Sigma^{+}$dos isotopômeros da molécula LiH. $D_{0}^{A}$ representa a profundidade do potencial algébrico (3.82) quando $\theta_{2}=2 j(2 j-1) / 4 . D_{e}^{A}$ representa a profundidade do potencial algébrico (3.82) quando $\theta_{2}=(j+1)^{2}$ ou $\theta_{2}=(j+1+\delta j)^{2}$ (correções). $D_{e}$ representa a altura do potencial RKR ou IPA, $D_{e}=V(r \rightarrow \infty)-V\left(R_{e}\right)$, onde $R_{e}$ é a posição de equilíbrio, e $D_{0}$ é a energia de dissociação, $D_{0}=D_{e}-E(0)$. Os percentuais na quarta e na sétima linhas são calculados em relação a $D_{0}$ e $D_{e}$, respectivamente. Todas as energias estão $\mathrm{em}^{-1}$.

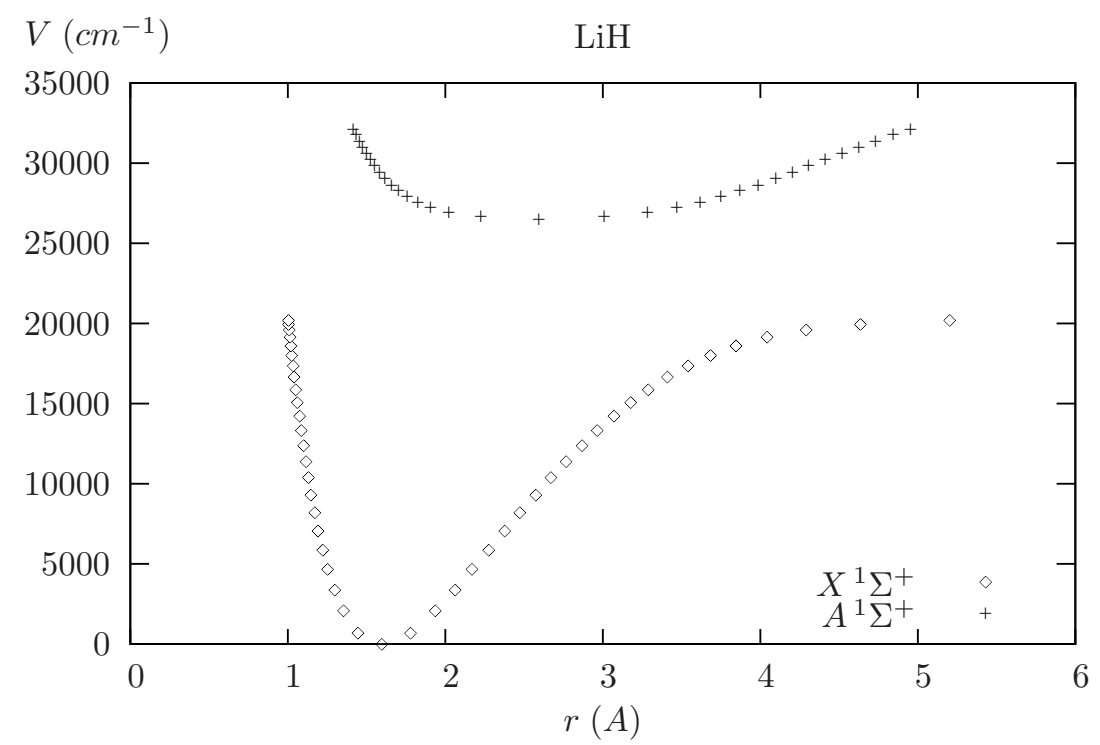

Figura 4.1: Curvas de potencial para os dois primeiros estados eletrônicos da molécula LiH. $X^{1} \Sigma^{+}$ representado o estado fundamental e $A^{1} \Sigma^{+}$o primeiro estado excitado. Estes potenciais foram calculados diretamente das energias vibracionais observadas pelo método IPA [38]. As respectivas constantes moleculares estão na Tabela 4.1. 


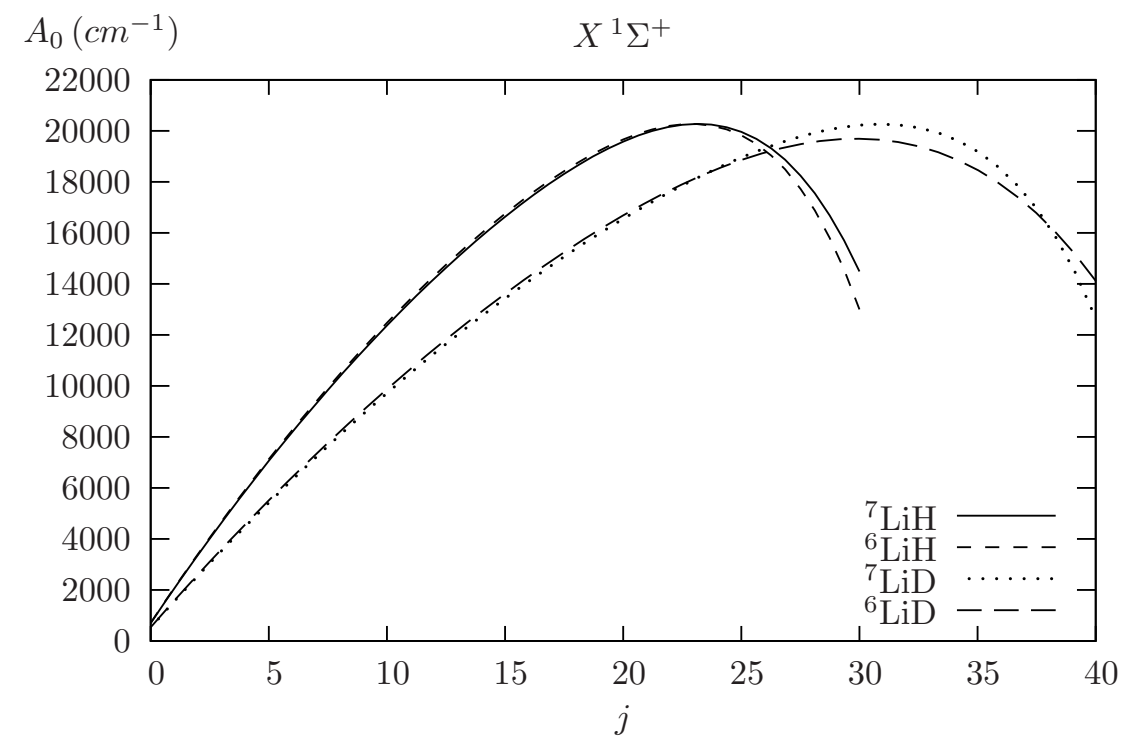

Figura 4.2: Parâmetro independente $A_{0}$ do hamiltoniano vibracional (3.51) (todas as potências) do estado eletrônico fundamental $X^{1} \Sigma^{+}$para os isotopômeros da molécula LiH. Neste modelo, o desvio médio é independente de $j$ e $A_{0}$ é um polinômio em $j$, o número quântico (adimensional) da representação irredutível da álgebra $\mathrm{su}(2)$. As otimizações foram realizadas com $n=9$ em (3.51), exceto para o isotopômero ${ }^{6} \mathrm{LiD}(n=5)$. Os valores máximos de $A_{0}$ estão próximos dos valores para as profundidades $D_{e}$ listadas na Tabela 4.1. Estes máximos ocorrem para valores de $j$ próximos dos valores previstos para o número máximo de níveis vibracionais, conforme indicado na Tabela 4.2. 


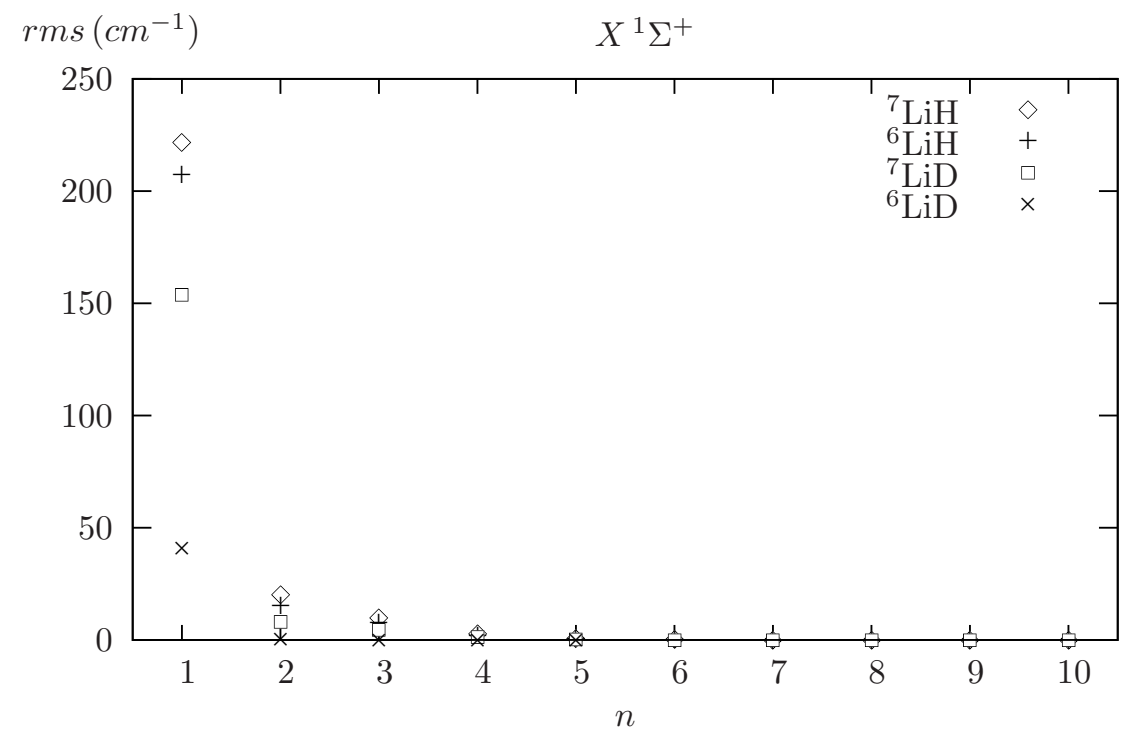

Figura 4.3: Desvio médio quadrático (3.57) em função do número de parâmetros $(n+1)$ para os isotopômeros da molécula LiH no estado eletrônico fundamental $X^{1} \Sigma^{+}$. O hamiltoniano algébrico utilizado inclui todas as potências em $J_{y}$, Eq. 3.51. Neste caso o desvio médio é independente de $j$, cujos valores em função do número de parâmetros estão mostrados na Figura 4.4.

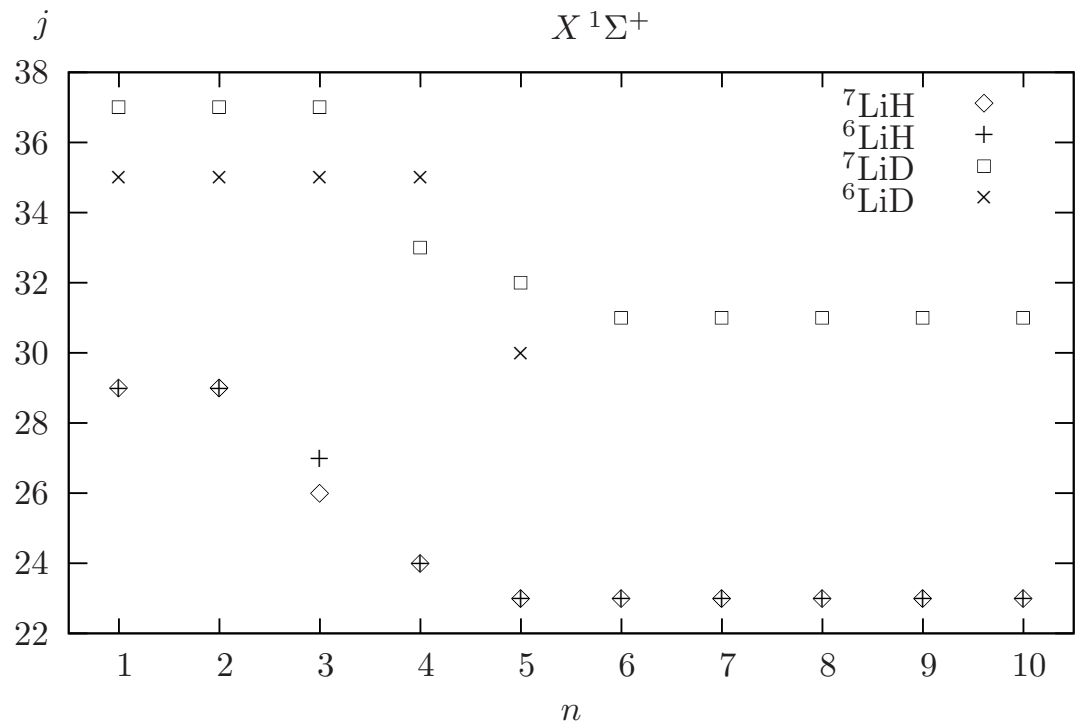

Figura 4.4: Valores otimizados de $j$ em função do número de parâmetros $(n+1)$ para os isotopômeros da molécula LiH no estado eletrônico fundamental $X^{1} \Sigma^{+}$. Estes valores otimizados convergem para o número de estados previstos por outros modelos. O hamiltoniano algébrico utilizado inclui todas as potências em $J_{y}$, Eq. 3.51. Os parâmetros lineares estão mostrados na Tabela 4.2. 


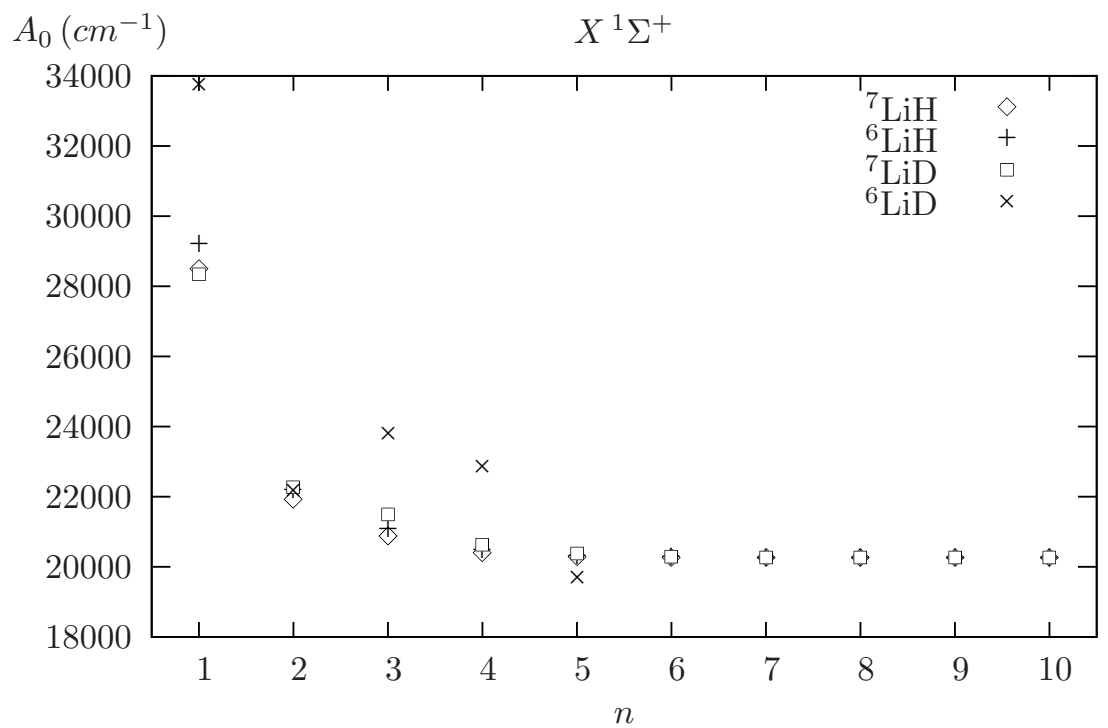

Figura 4.5: Parâmetro independente $A_{0}$ do hamiltoniano algébrico 3.51 (todas as potências) em função do número de parâmetros $(n+1)$ para os isotopômeros da molécula LiH no estado eletrônico fundamental $X^{1} \Sigma^{+}$. Estes valores foram calculados com os valores otimizados de $j$ mostrados na Figura 4.4. Os demais parâmetros estão mostrados na Tabela 4.2. 


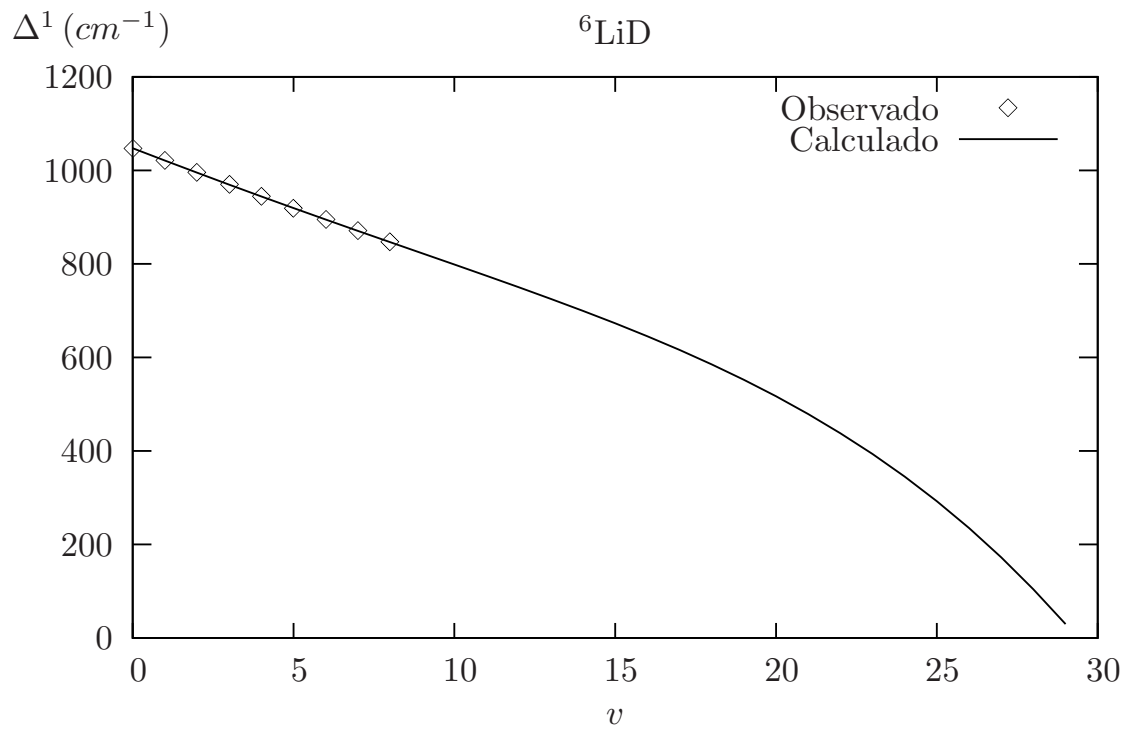

Figura 4.6: Diferença sucessiva de primeira ordem $\Delta^{1}$ (Birge-Sponer) para as energias vibracionais observadas (pontos) e calculadas (linha cheia), etiquetadas por $v$, do isotopômero ${ }^{6} \mathrm{LiD}$ no estado eletrônico fundamental $X^{1} \Sigma^{+}$. As energias vibracionais teóricas foram calculadas usando os parâmetros mostrados na Tabela 4.2. $\mathrm{O}$ valor de $j$ em $\Delta^{1}=0$ coincide com o valor previsto para o número máximo de estados vibracionais. O desvio da linha cheia em relação a uma reta mostra o efeito de anarmonicidades embutidas no modelo algébrico 3.51 (todas as potências). As diferenças sucessivas de segunda e terceira ordem estão mostradas nas Figuras 4.7-4.8. As diferenças sucessivas normalizadas, até terceira ordem, para os demais isotopômeros estão mostradas nas Figuras 4.9-4.12. 


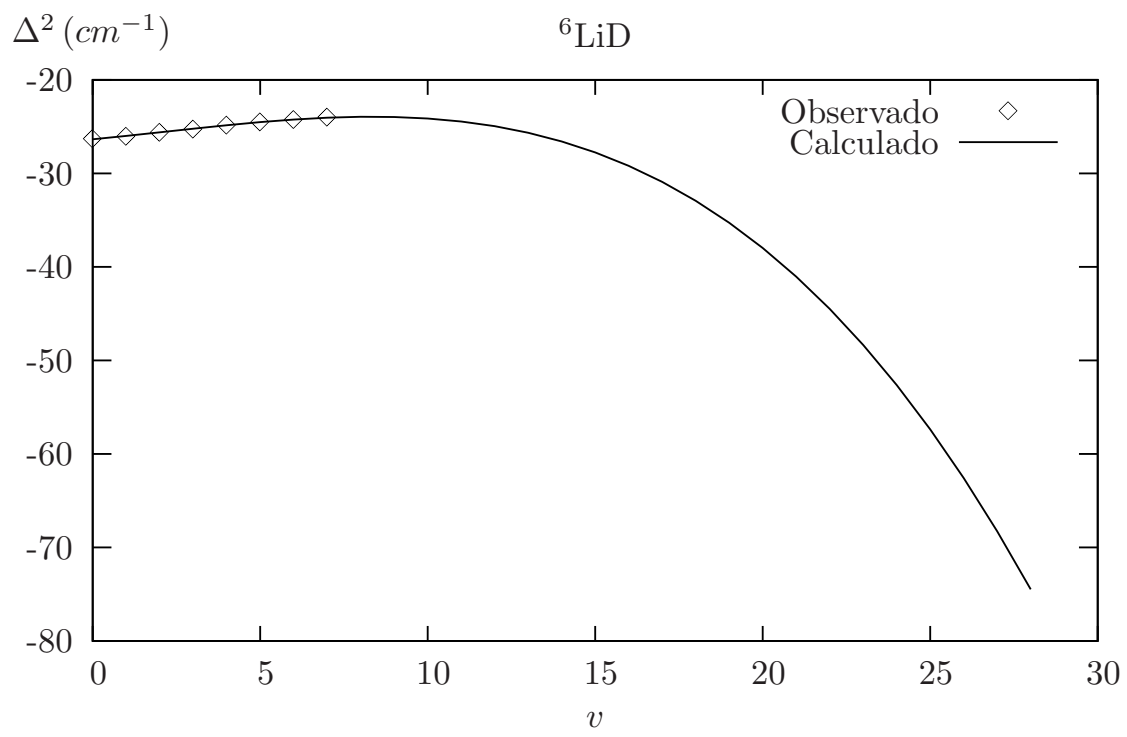

Figura 4.7: Diferença sucessiva de segunda ordem $\Delta^{2}$ (Birge-Sponer) para as energias vibracionais observadas (pontos) e calculadas (linha cheia), etiquetadas por $v$, do isotopômero ${ }^{6} \mathrm{LiD}$ no estado eletrônico fundamental $X^{1} \Sigma^{+}$. As energias vibracionais teóricas foram calculadas usando os parâmetros mostrados na Tabela 4.2. O modelo algébrico utilizado inclui todas as potências, Eq. 3.51. As diferenças sucessivas de primeira e terceira ordem estão mostradas nas Figuras 4.6 e 4.8, respectivamente. As diferenças sucessivas normalizadas, até terceira ordem, para os demais isotopômeros estão mostradas nas Figuras 4.9-4.12. 


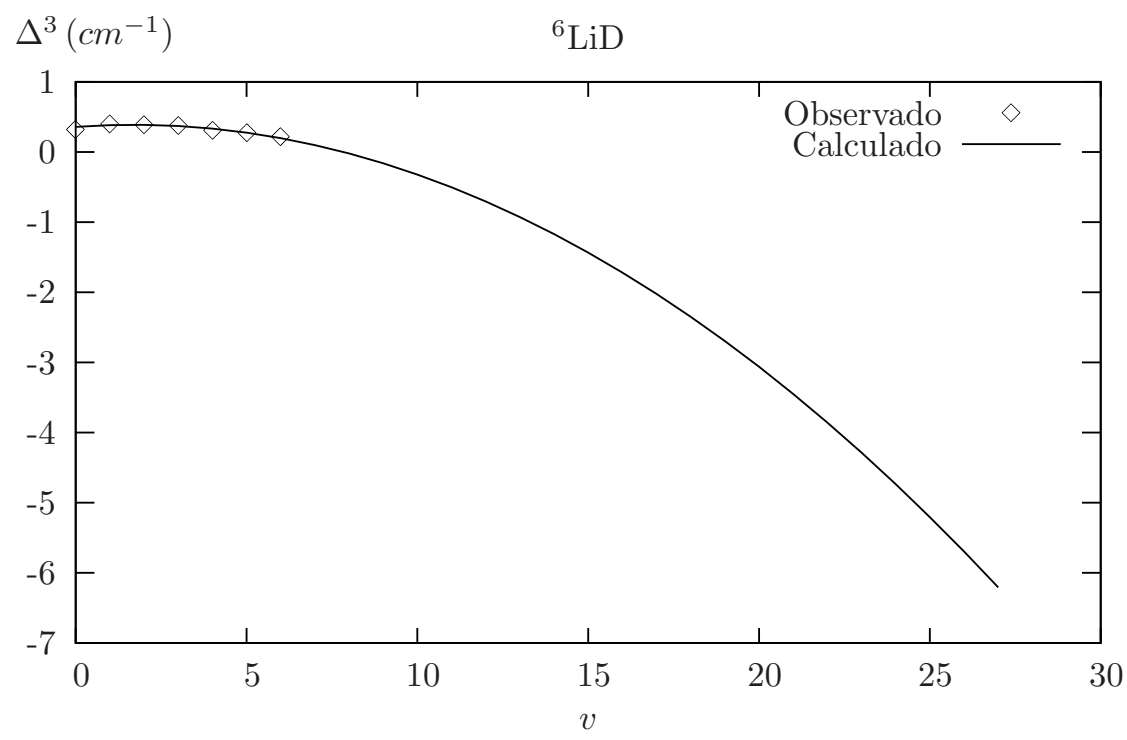

Figura 4.8: Diferença sucessiva de terceira ordem $\Delta^{3}$ (Birge-Sponer) para as energias vibracionais observadas (pontos) e calculadas (linha cheia), etiquetadas por $v$, do isotopômero ${ }^{6} \mathrm{LiD}$ no estado eletrônico fundamental $X^{1} \Sigma^{+}$. As energias vibracionais teóricas foram calculadas usando os parâmetros mostrados na Tabela 4.2. O modelo algébrico utilizado inclui todas as potências, Eq. 3.51. As diferenças sucessivas de primeira e segunda ordem estão mostradas nas Figuras 4.7-4.8. As diferenças sucessivas normalizadas, até terceira ordem, para os demais isotopômeros estão mostradas nas Figuras 4.9-4.12.

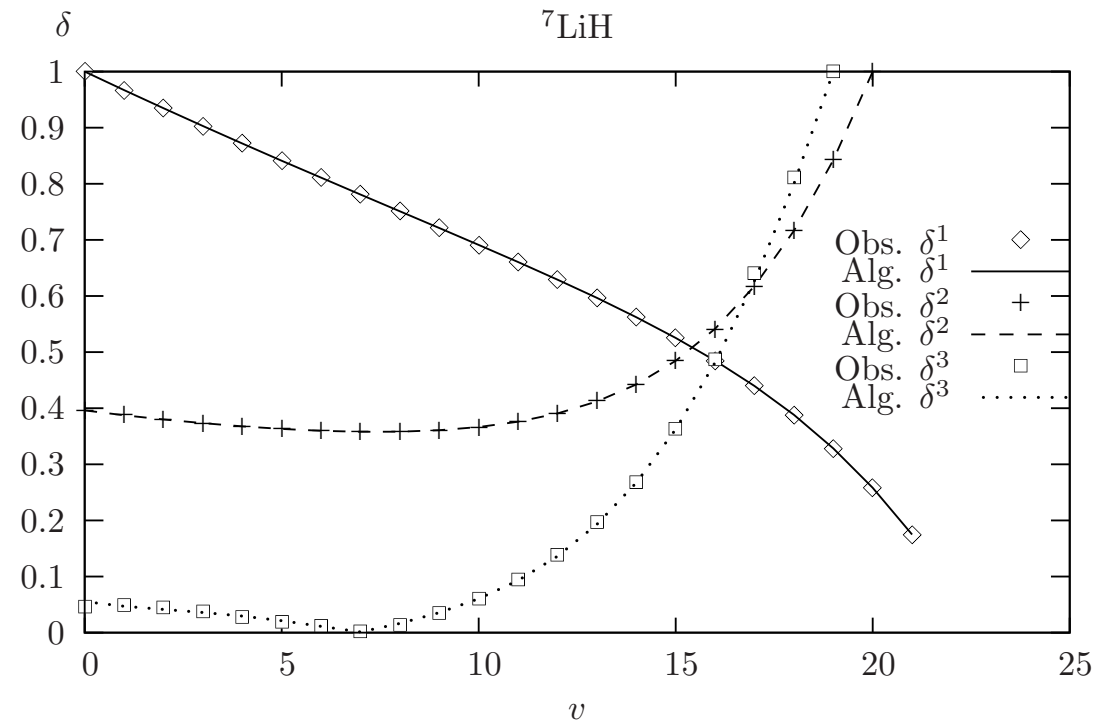

Figura 4.9: Diferenças sucessivas normalizadas $(\delta)$, até terceira ordem, entre as energias vibracionais observadas (pontos) e calculadas (linhas), etiquetadas por $v$, para a molécula ${ }^{7} \mathrm{LiH}$ no estado eletrônico fundamental $X^{1} \Sigma^{+}$. As energias vibracionais teóricas foram calculadas usando modelo algébrico 3.51 (todas as potências) e os parâmetros mostrados na Tabela 4.2. 




Figura 4.10: Diferenças sucessivas normalizadas $(\delta)$, até terceira ordem, entre as energias vibracionais observadas (pontos) e calculadas (linhas), etiquetadas por $v$, para a molécula ${ }^{7}$ LiD no estado eletrônico fundamental $X^{1} \Sigma^{+}$. As energias vibracionais teóricas foram calculadas usando modelo algébrico 3.51 (todas as potências) e os parâmetros mostrados na Tabela 4.2.

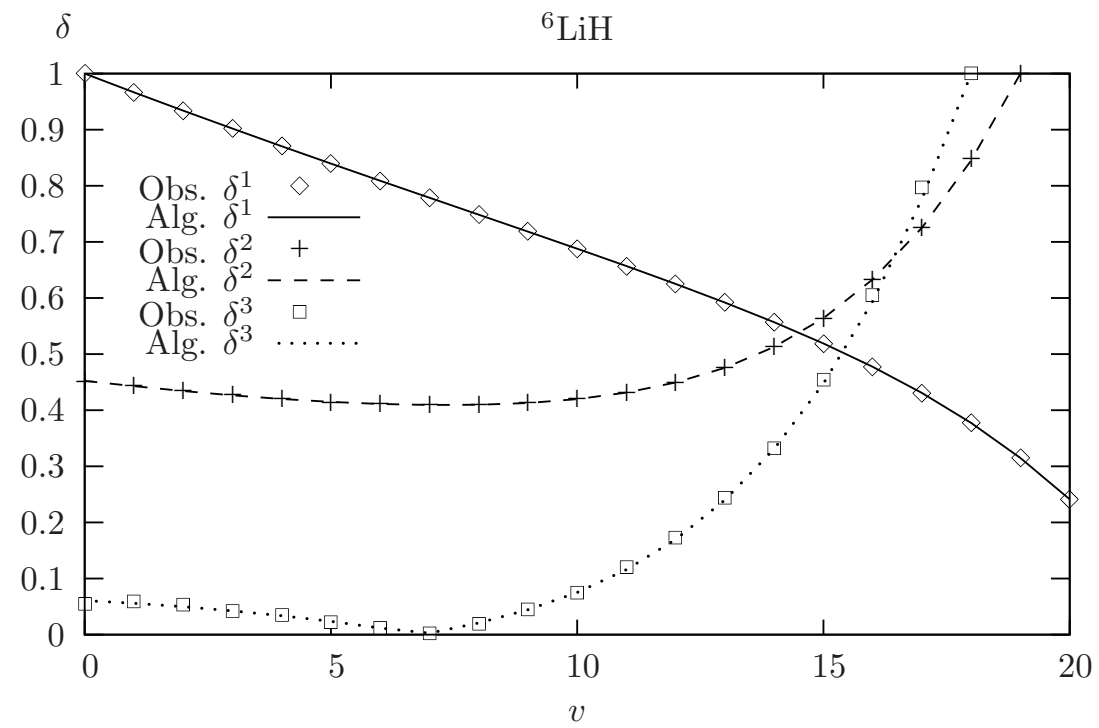

Figura 4.11: Diferenças sucessivas normalizadas $(\delta)$, até terceira ordem, entre as energias vibracionais observadas (pontos) e calculadas (linhas), etiquetadas por $v$, para a molécula ${ }^{6} \mathrm{LiH}$ no estado eletrônico fundamental $X^{1} \Sigma^{+}$. As energias vibracionais teóricas foram calculadas usando modelo algébrico 3.51 (todas as potências) e os parâmetros mostrados na Tabela 4.2. 


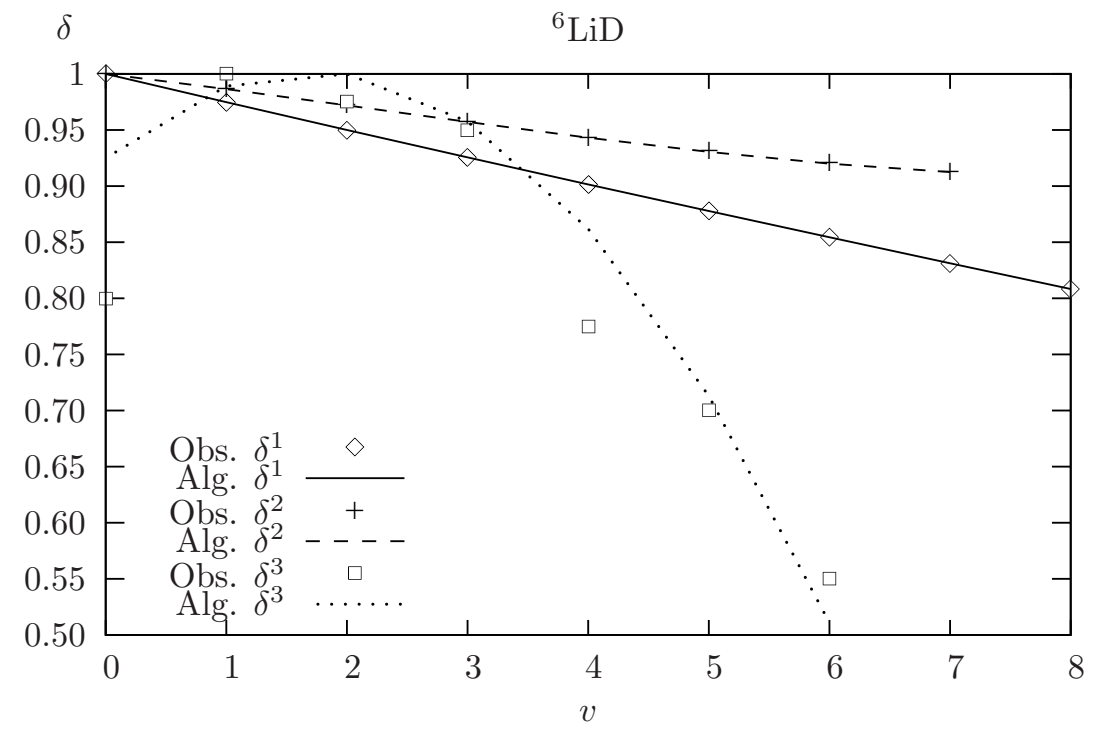

Figura 4.12: Diferenças sucessivas normalizadas $(\delta)$, até terceira ordem, entre as energias vibracionais observadas (pontos) e calculadas (linhas), etiquetadas por $v$, para a molécula ${ }^{6} \mathrm{LiD}$ no estado eletrônico fundamental $X^{1} \Sigma^{+}$. As energias vibracionais teóricas foram calculadas usando modelo algébrico 3.51 (todas as potências) e os parâmetros mostrados na Tabela 4.2. Conforme indicado na Tabela 4.2, devido à falta de mais energias vibracionais observadas, a otimização foi feita com poucos parâmetros $(n=5)$. Isto acarreta as discrepâncias na diferença sucessiva de terceira ordem. 


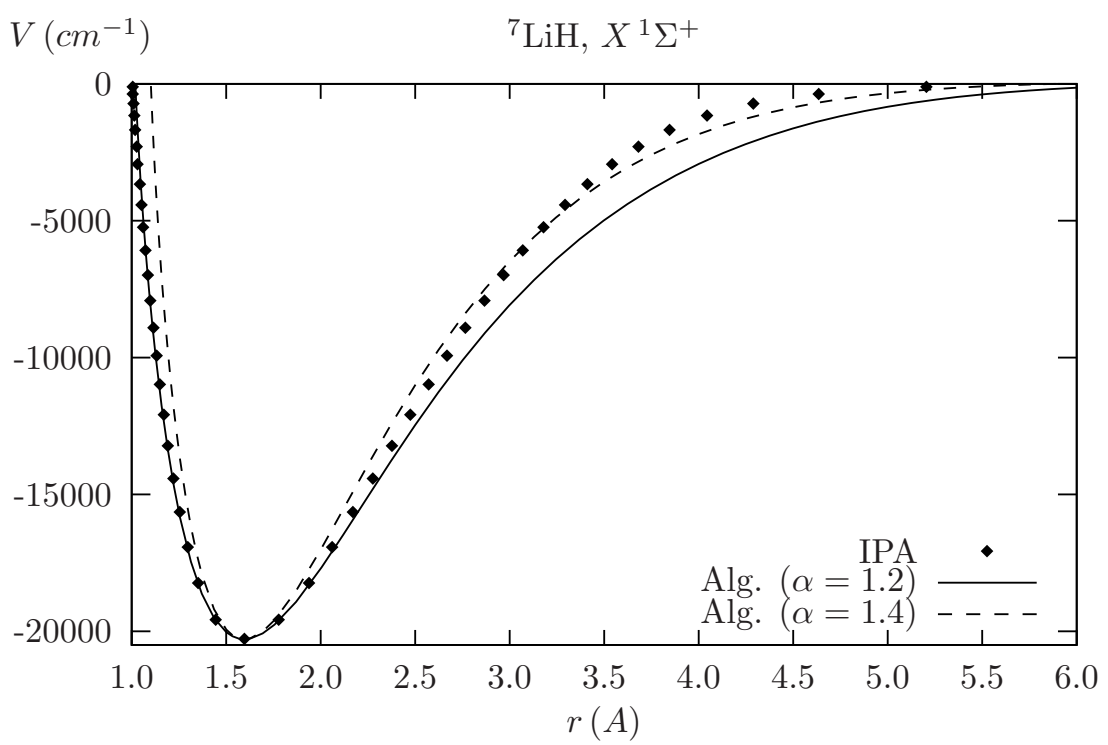

Figura 4.13: Curvas de potencial, algébricas (linhas, Eq. (3.81)) e IPA (pontos, Ref. [38]), para o estado eletrônico fundamental $X^{1} \Sigma^{+}$da molécula ${ }^{7} \mathrm{LiH}$. Os ajustes na profundidade do potencial algébrico estão listados na Tabela 4.5. Os parâmetros do modelo algébrico 3.51 (todas as potências) estão nas Tabelas 4.1 e 4.2. A distância internuclear $r$ está em Angstrons e o alcance $\alpha$ do potencial algébrico (3.81) está em inversos de Angstrons.

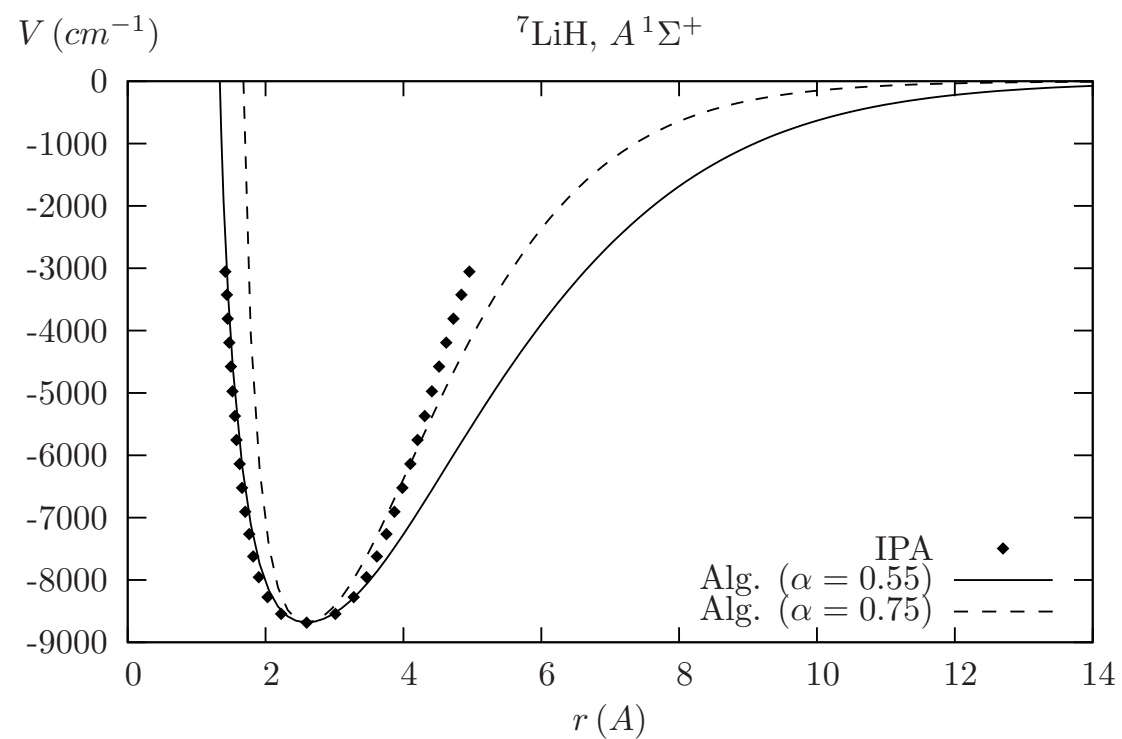

Figura 4.14: Curvas de potencial, algébricas (linhas, Eq. (3.82)) e IPA (pontos, Ref. [38]), para o primeiro estado eletrônico excitado $A^{1} \Sigma^{+}$da molécula ${ }^{7} \mathrm{LiH}$. Os ajustes na profundidade do potencial algébrico estão listados na Tabela 4.8. Os parâmetros do modelo algébrico 3.52 (potências pares) estão nas Tabelas 4.1 e 4.6. A distância internuclear $r$ está em Angstrons e o alcance $\alpha$ do potencial algébrico (3.82) está em inversos de Angstrons. 


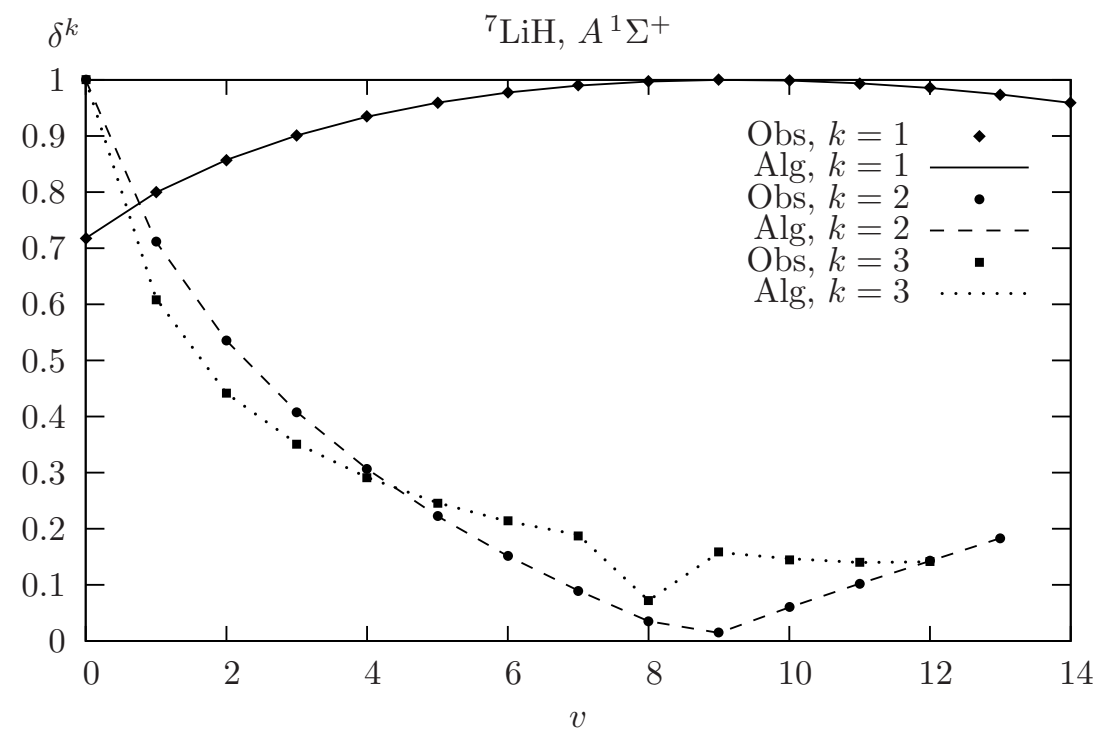

Figura 4.15: Diferença sucessiva de primeira ordem $\Delta^{1}$ (Birge-Sponer) entre as energias vibracionais observadas (pontos) e calculadas (linhas), etiquetadas por $v$, para os isotopômeros da molécula LiH no primeiro estado eletrônico excitado $A^{1} \Sigma^{+}$. As energias vibracionais teóricas foram calculadas usando os parâmetros mostrados na Tabela 4.6. Os valores de $j$ em $\Delta^{1}=0$ coincidem com os valores previstos para o número máximo de estados vibracionais. O desvio das linhas em relação a uma reta mostra o efeito de anarmonicidades embutidas no modelo algébrico 3.52 (potências pares). As diferenças sucessivas normalizadas, até terceira ordem, para o isotopômero estão mostradas na Figura 4.16. 


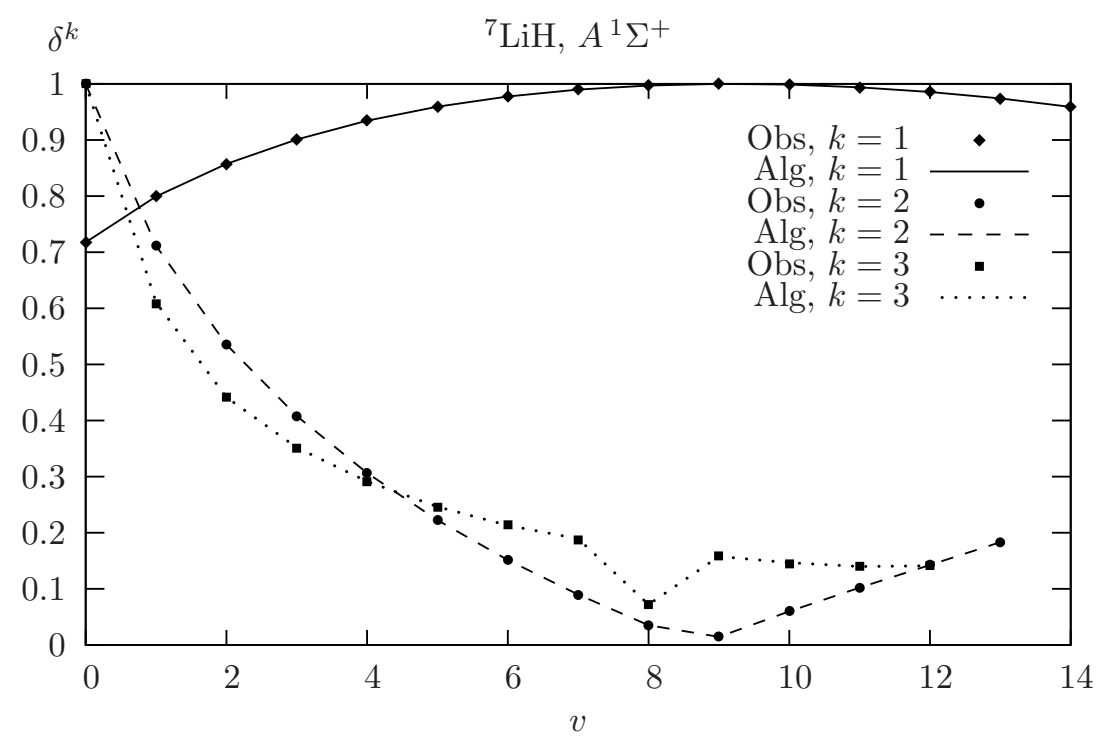

Figura 4.16: Diferenças sucessivas normalizadas $\left(\delta^{k}\right)$, até terceira ordem, entre as energias vibracionais observadas (pontos) e calculadas (linhas), etiquetadas por $v$, para a molécula ${ }^{7} \mathrm{LiH}$ no primeiro estado eletrônico excitado $A^{1} \Sigma^{+}$. As energias vibracionais do hamiltoniano efetivo (3.52) foram calculadas usando os parâmetros mostrados na Tabela 4.6. 


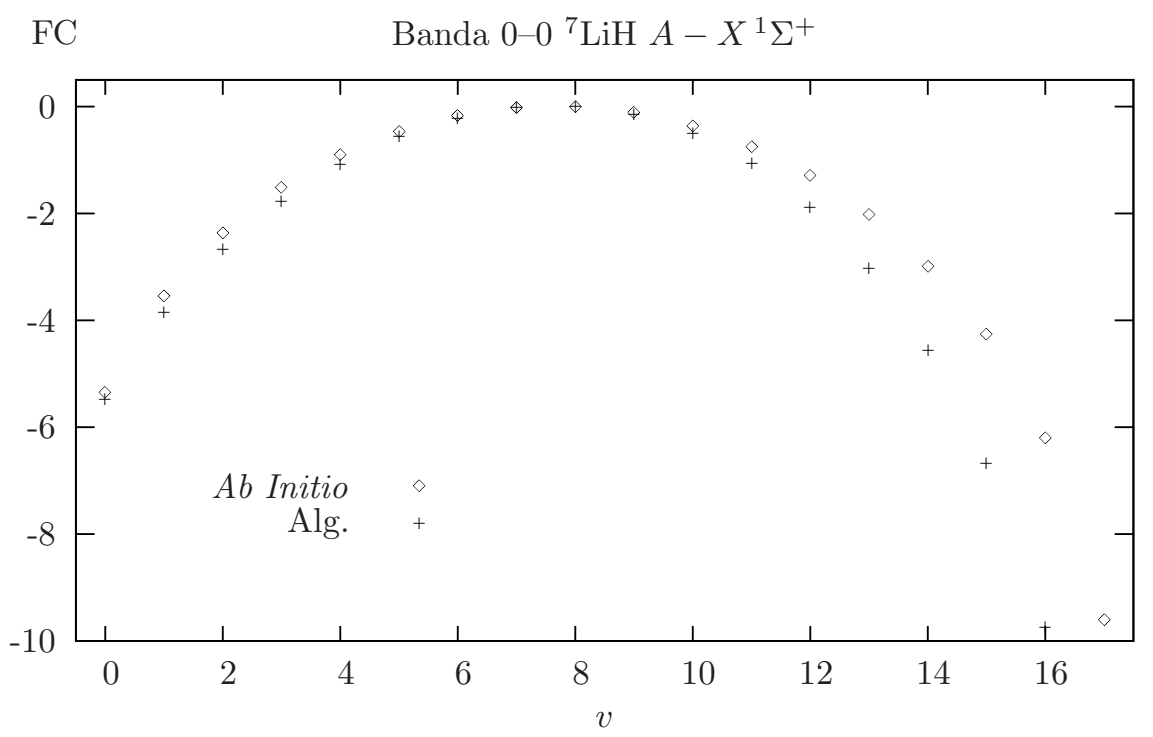

(a) Fatores de Franck-Condon para a banda 0-0.

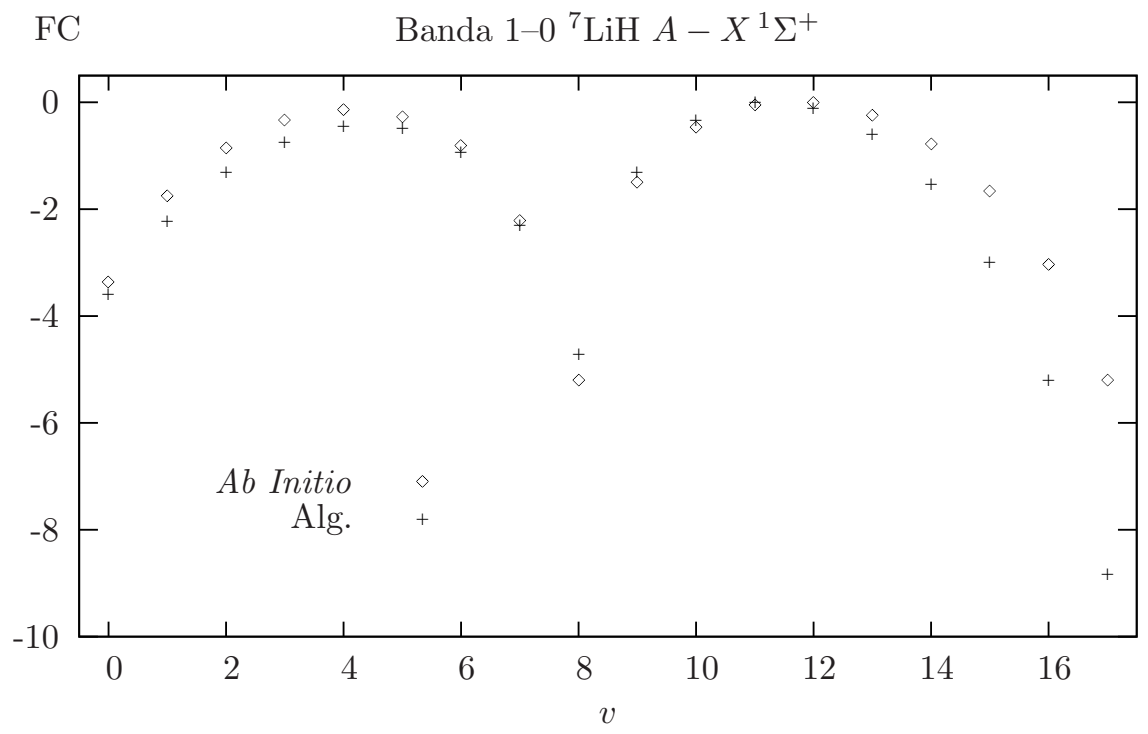

(b) Fatores de Franck-Condon para a banda 1-0.

Figura 4.17: Fatores de Franck-Condon adimensionais, algébricos (Eq. (3.94)) e ab initio (Ref. 4.14), para as bandas 0-0 e 1-0 entre os estados eletrônicos $A-X^{1} \Sigma^{+}$da molécula ${ }^{7} \mathrm{LiH}$. As constantes adimensionais definidas em (3.95) são $\tau=0.51$ e $\gamma=0.40$. O número quântico $v$ é das energias vibracionais do estado eletrônico fundamental $X^{1} \Sigma^{+}$. 


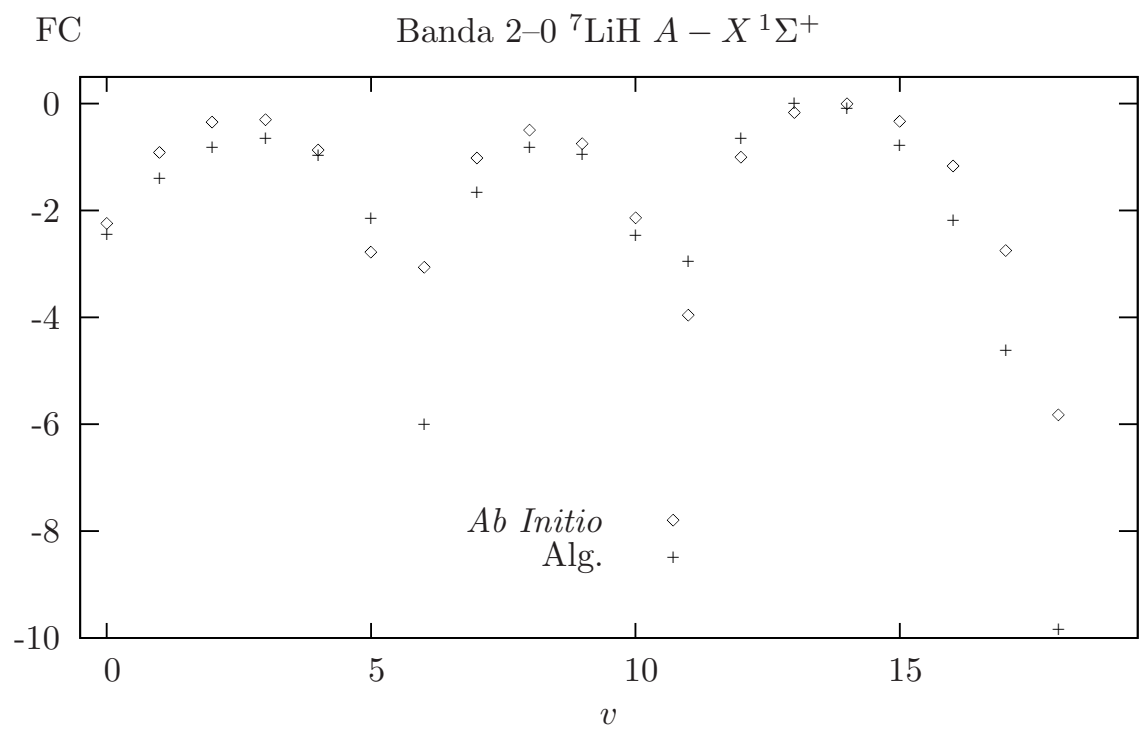

(a) Fatores de Franck-Condon para a banda 2-0



(b) Fatores de Franck-Condon para a banda 3-0.

Figura 4.18: Fatores de Franck-Condon adimensionais, algébricos (Eq. (3.94)) e ab initio (Ref. 4.14), para as bandas 2-0 e 3-0 entre os estados eletrônicos $A-X^{1} \Sigma^{+}$da molécula ${ }^{7} \mathrm{LiH}$. As constantes adimensionais definidas em (3.95) são $\tau=0.51$ e $\gamma=0.40$. O número quântico $v$ é das energias vibracionais do estado eletrônico fundamental $X^{1} \Sigma^{+}$. 


\section{Capítulo 5}

\section{Conclusões e perspectivas}

Podemos resumir o conteúdo desta dissertação em quatro partes: (1) a construção de hamiltonianos vibracionais com base na álgebra de momentum angular $\mathrm{su}(2) ;(2)$ a construção de potenciais vibracionais via estados coerentes do grupo SU(2); (3) o cálculo de fatores de Franck-Condon; e (4) aplicações usando moléculas diatômicas alcalinas, feitas aqui pela primeira vez. As seções seguintes apresentam nossas considerações finais e perspectivas futuras decorrentes deste trabalho.

\subsection{Modelos algébricos}

Nós usamos uma das relações conhecidas entre o potencial de Morse unidimensional e a álgebra su(2) como protótipo para o estabelecimento de modelos algébricos unidimensionais capazes de gerar espectros vibracionais de sistemas diatômicos de forma acurada. Neste protótipo, o espectro vibracional do potencial de Morse unidimensional é obtido com um hamiltoniano puramente quadrático em $J_{y}$. Assim, esperamos que o hamiltoniano algébrico efetivo possa ser construído como um polinômio em $J_{y}$, com constantes arbitrárias. Isto torna possível a diagonalização deste hamiltoniano no espaço das representações irredutíveis da álgebra $\mathrm{su}(2)$, formado pelos autovetores de $J^{2}$ e $J_{y}$. Vários modelos algébricos podem então ser criados, diferindo ligeiramente nos seguintes aspectos: (1) a álgebra su(2) pode ser deformada $(q \neq 1)$ ou não $(q=1)$ e $(2)$ o hamiltoniano efetivo pode conter todas as potências em $J_{y}$, um dos três geradores da álgebra su(2), ou somente as potências pares em $J_{y}$.

\subsubsection{Modelos com deformação $(q \neq 1)$}

Embora não esteja detalhado no capítulo anterior, fizemos também um estudo aplicado à molécula LiH usando hamiltonianos algébricos deformados, isto é, usando elementos da álgebra deformada $\mathrm{su}_{\mathrm{q}}(2)$. Os resultados mostram que não há, em nenhum caso estudado, valores reais para o parâmetro de deformação $q$ onde o desvio médio quadrático torna-se menor que o desvio médio quadrático correspondente ao caso clássico. Há um pequeno indício de melhoria, muito singela, para o caso onde $q=\exp (i \omega), \omega \in \mathbb{R}$ (círculo unitário), mas não foi possível discernir se esta melhoria foi um resultado puramente devido ao parâmetro $q$ ou se foi apenas uma conseqüência de termos introduzido mais um parâmetro no modelo. Portanto, o espectro vibracional não é um teste decisivo para sabermos se álgebras deformadas têm algum papel relevante nestes modelos. Outros testes devem ser desenhados. Por exemplo, mesmo que a descrição das energias vibracionais não se alterem com a introdução de deformações, elas poderão dar contribuições importantes aos fatores de Franck-Condon e aos potenciais. Infelizmente, ainda temos problemas de convergência com o cálculo das integrais envolvidas devido às deformações produzirem potências muito elevadas nas funções deformadas (caso real e no círculo unitário) [64, 63]. Isto nos impede de calcular os fatores de Franck-Condon com eficiência neste momento. Também não dispomos até o momento de estados coerentes deformados apropriados para a construção de potenciais vibracionais deformados. O problema maior com os estados coerentes deformados existente [71] está relacionado com forma do elemento de volume (medida) usado na resolução da unidade: em todos os casos conhecidos há 
o envolvimento de funções deformadas, as quais são muito inconvenientes em cálculos numéricos. Estes dois problemas estão sob investigação e deverão ser resolvidos em trabalhos futuros.

\subsubsection{Caso sem deformação $(q=1)$}

Trabalhamos em detalhes com dois modelos algébricos unidimensionais clássicos (sem deformação) aplicados às moléculas diatômicas alcalinas $\mathrm{LiH}$ e $\mathrm{Li}_{2}$. Para os sistemas heterogêneos, um dos modelos, envolvendo todas as potências em $J_{y}$, mostrou-se mais adequado ao estado eletrônico fundamental $X^{1} \Sigma$, enquanto que o outro modelo, envolvendo apenas as potências pares em $J_{y}$, mostrou-se mais adequado ao primeiro estado eletrônico excitado $A^{1} \Sigma$. Para os sistemas homogêneos, podemos afirmar que as energias vibracionais dos dois primeiros estados eletrônicos admitem uma descrição algébrica com um modelo único, envolvendo todas as potências em $J_{y}$.

Vale mencionar que usamos o gerador $J_{y}$ para construir os hamiltonianos efetivos. No entanto, teremos exatamente os mesmos resultados para o espectro vibracional usando qualquer um dos outros dois geradores: $J_{x}$ e $J_{z}$. Diferenças ocorrerão apenas quando usarmos os valores médios destes geradores, por exemplo, na reconstrução dos potenciais vibracionais.

\section{Espectro vibracional}

Em todos os casos, as energias vibracionais, contidas no intervalo entre $100 \mathrm{~cm}^{-1}$ e $20000 \mathrm{~cm}^{-1}$, podem ser geradas algebricamente de forma acurada, com um desvio médio em torno de $10^{-3} \mathrm{~cm}^{-1}$. As anarmonicidades destes espectros vibracionais, isto é, o desvio da previsão harmônica, é da ordem de $1000 \mathrm{~cm}^{-1}$. Esta forma acurada é visivelmente verificada pela perfeita concordância entre as diferenças sucessivas das energias vibracionais calculadas e observadas, até terceira ordem. Verificamos também que nossas previsões para o número total de estados vibracionais concordam muito bem com as previsões feitas por outros modelos. Em geral, estas previsões feitas com o modelo algébrico são confirmadas através do mínimo do desvio médio e pela profundidade dos potenciais vibracionais. Portanto, estas previsões são confirmadas de duas formas independentes.

Do ponto de vista computacional, a otimização de todos os parâmetros independentes do modelo algébrico é feita completamente em um ambiente de computação simbólica onde a precisão dos valores encontrados para estas constantes é arbitrária. Desta forma, não tivemos problemas de instabilidades numéricas relacionados à determinação destes parâmetros, como reportado em outros trabalhos [75].

\section{Potenciais vibracionais}

A obtenção de potenciais a partir das energias vibracionais ("problema inverso") é um problema antigo e muito importante em espectroscopia molecular, bem como em outras áreas. Mostramos aqui uma solução baseada nos estados coerentes do grupo SU(2), o grupo de Lie associado à álgebra su(2). Nossos estados coerentes foram calculados explicitamente, sem qualquer aproximação. Mesmo assim, precisamos introduzir correções para obtermos corretamente a profundidade destes potenciais previstas em outros modelos. No entanto, estas correções obedecem um padrão muito simples: (1) sem correções, a profundidade dos potenciais algébricos tendem à energia de dissociação (profundidade menos a energia do primeiro estado vibracional) e (2) as correções são pequenas. O fato de reproduzirmos as principais características dos potenciais vibracionais para os dois primeiros estados eletrônicos usando os mesmos parâmetros obtidos na construção do hamiltoniano efetivo, indicam que este hamiltoniano algébrico tem um conteúdo físico condizente com o problema em questão. Vimos também que o alcance dos potenciais vibracionais algébricos diferem ligeiramente do alcance dos potenciais obtidos por métodos ab initio. Iremos, em um trabalho futuro, verificar o efeito desta diferença nos fatores de Franck-Condon calculados diretamente através das auto-funções destes potenciais algébricos.

\section{Fatores de Franck-Condon}

Nós calculamos os fatores de Franck-Condon explicitamente, usando as funções de Morse como autofunções do hamiltoniano efetivo. Fizemos todas as integrais numericamente em Fortran. As instabilidades numéricas foram minimizadas drasticamente usando somente uma forma recursiva para calcular as funções de Laguerre, evitando assim a necessidade de realizar qualquer aproximação nas funções de 
Morse. Infelizmente, não dispomos de informações quantitativas retiradas diretamente dos experimentos para os fatores de Franck-Condon. No entanto, nossos resultados concordam com outros cálculos na região de baixa energia e concordam também com as observações experimentais qualitativas.

\subsection{Trabalhos futuros}

Existe uma quantidade enorme de estudos futuros que pode ser realizada no contexto dos modelos algébricos discutidos aqui. Alguns destes estudos são imediatos, outros poderão requerer trabalhos mais complexos.

De imediato, pode ser realizado um trabalho comparativo envolvendo outras moléculas diatômicas, além dos sistemas alcalinos estudados nesta dissertação, usando-se as mesmas ferramentas utilizadas aqui. Este estudo comparativo servirá para estabelecermos se há ou não a presença de uma universalidade no modelo algébrico. Vimos aqui, que existe uma preferência pelos sistemas heterogêneos alcalinos por um determinado modelo algébrico. A questão é saber se esta preferência será mantidas para moléculas nãoalcalinas. Se for mantida, é possível estabelecer alguma correlação entre modelos algébricos e propriedades microscópicas destes sistemas?

Outro trabalho também imediato é um estudo do espectro vibracional dos estados eletrônicos mais excitados. Neste caso, pode ocorrer repulsões entre níveis eletrônicos (anti-crossings) e mesmo uma falha sensível na aproximação de núcleos fixos. O comportamento dos modelos algébricos nestas condições extremas ainda é completamente desconhecido.

A obtenção de potenciais vibracionais diatômicos através dos métodos semi-clássicos RKR e IPA (ou variantes) é, sem dúvidas, muito eficiente, pois fornece todas as propriedades físicas importantes destes sistema com um baixo custo computacional. No entanto, esta situação muda drasticamente em sistemas moleculares ligeiramente maiores, como moléculas tri e tetra-atômicas. Nestas casos, os potenciais serão superfícies de dimensões altas contendo um número grande de parâmetros livres a serem otimizados. Neste caso, como mostram algumas análises recentes [30], os modelos algébricos unidimensionais podem ser muito úteis, pois permitem um tratamento dos principais acoplamentos numa forma mais simples. Estes trabalhos futuros deverão envolver outras formas de calcular os potenciais algébricos, a fim de evitar a necessidade de correções na profundidade dos mesmos. Portanto, estes trabalhos serão mais complexos. Além disto, estes estudos poderão adicionar informações sobre a universalidade destes potenciais vibracionais.

Naturalmente, após estes estudos com sistemas diatômicos, iremos passar ao estudo de sistemas moleculares maiores (tri-atômicos e tetra-atômicos). Vale mencionar que, em geral, o desempenho dos modelos algébricos em moléculas diatômicas é mais dispendioso do que o desempenho dos mesmos aplicados a sistemas moleculares maiores. Em parte, isto se deve ao fato de ser desnecessário o uso de potências altas nos hamiltonianos vibracionais de sistemas maiores. Em muitos casos basta um hamiltoniano polinomial de grau dois ou três (compare com a necessidade de um hamiltoniano de grau oito para moléculas diatômicas). Complicações computacionais aparecem no processo de otimização devido à necessidade de usarmos termos não-diagonais no hamiltoniano efetivo. Estes termos não-diagonais são importantes para introduzir efeitos não-lineares específicos, bem como para garantir a simetria correta das funções de onda, impostas pelas simetrias discretas (caso estejam presentes no sistema molecular). Em casos onde não estamos preocupados com as simetrias discretas, podemos utilizar apenas termos diagonais no hamiltoniano vibracional $[95,96,97,98]$. Muitos destes termos diagonais serão suficientes para reproduzir mais de $90 \%$ dos acoplamentos que poderão ocorrer entre os modos normais de vibração.

Para encerrar, observamos que estes estudos com moléculas diatômicas e tri-atômicas não são importantes apenas para o desenvolvimento natural de modelos algébricos aplicados a sistemas moleculares maiores. Estes sistemas moleculares pequenos são fundamentais, seja diretamente, ou como sítios ativos em sistemas moleculares maiores, ou como subprodutos, em questões ambientais de interesse global, como o efeito estufa, poluição atmosférica, produção de água potável, etc. Hoje, graças ao desenvolvimento de técnicas novas em espectroscopia molecular, temos indícios evidentes da importância de termos descrições detalhadas, precisas, dos espectros moleculares destes sistemas pequenos para compreendermos em detalhes os mecanismos básicos envolvidos no efeito estufa. Além, das questões ambientais, sistemas moleculares pequenos também são importantes como objetos de estudo em espectroscopia em tempo real. 


\section{Apêndice A}

\section{Valores médios de $J_{x}$ e $J_{y}$}

Vamos iniciar calculando explicitamente os valores médios de $J_{x}$ e $J_{y}$,

$$
J_{x}=\frac{1}{2}\left(J_{+}+J_{-}\right), \quad J_{y}=\frac{1}{2 i}\left(J_{+}-J_{-}\right) .
$$

Devido à presença de constantes diferentes nestas duas expressões, vamos calcular inicialmente o valor médio de $J_{+} \pm J_{-}$:

$$
\begin{aligned}
\left\langle J_{+} \pm J_{-}\right\rangle & =\frac{x^{j}}{(1+x)^{2 j}} \sum_{m^{\prime}=j}^{-j} \sum_{m=j}^{-j} \tau^{* m^{\prime}} \tau^{m}\left(\begin{array}{c}
2 j \\
j+m^{\prime}
\end{array}\right)^{\frac{1}{2}}\left(\begin{array}{c}
2 j \\
j+m
\end{array}\right)^{\frac{1}{2}}\left\langle m^{\prime}\left|J_{+} \pm J_{-}\right| m\right\rangle \\
& =\frac{x^{j}}{(1+x)^{2 j}} \sum_{m^{\prime}=j}^{-j} \sum_{m=j}^{-j} \tau^{* m^{\prime}} \tau^{m}\left(\begin{array}{c}
2 j \\
j+m^{\prime}
\end{array}\right)^{\frac{1}{2}}\left(\begin{array}{c}
2 j \\
j+m
\end{array}\right)^{\frac{1}{2}} \\
& \times\left[\sqrt{\left(j-m^{\prime}\right)\left(j+m^{\prime}+1\right)}\left\langle m^{\prime}-1 \mid m\right\rangle \pm \sqrt{(j-m)(j+m+1)}\left\langle m^{\prime} \mid m-1\right\rangle\right] .
\end{aligned}
$$

Como este último termo é simétrico na troca de $m$ por $m^{\prime}$, então

$$
\begin{aligned}
\left\langle J_{+} \pm J_{-}\right\rangle & =\frac{x^{j}}{(1+x)^{2 j}} \sum_{m^{\prime}=j}^{-j+1} \sum_{m=-j}^{j-1}\left(\tau^{* m^{\prime}} \tau^{m} \pm \tau^{* m} \tau^{m^{\prime}}\right)\left(\begin{array}{c}
2 j \\
j+m
\end{array}\right)^{\frac{1}{2}}\left(\begin{array}{c}
2 j \\
j+m^{\prime}-1
\end{array}\right)^{\frac{1}{2}}\left(j-m^{\prime}+1\right)\left\langle m^{\prime}-1 \mid m\right\rangle \\
& =\frac{x^{j}}{(1+x)^{2 j}} \sum_{m^{\prime}=-j}^{j-1} \sum_{m=-j}^{j-1}\left(\tau^{* m^{\prime}+1} \tau^{m} \pm \tau^{* m} \tau^{m^{\prime}+1}\right)\left(\begin{array}{c}
2 j \\
j+m
\end{array}\right)^{\frac{1}{2}}\left(\begin{array}{c}
2 j \\
j+m^{\prime}
\end{array}\right)^{\frac{1}{2}}\left(j-m^{\prime}\right)\left\langle m^{\prime} \mid m\right\rangle \\
& =\frac{\left(\tau^{*} \pm \tau\right)}{(1+x)^{2 j}} \sum_{m=-j}^{j-1}\left(\begin{array}{c}
2 j \\
j+m
\end{array}\right)(j-m) x^{j+m}=2 j \frac{\tau^{*} \pm \tau}{1+x}
\end{aligned}
$$

Desta forma, usando as variáveis canônicas (2.41) e as relações apresentadas em (2.55), os valores médios de $J_{x}$ e $J_{y}$ podem ser escritos como

$$
\left\langle J_{x}\right\rangle=+\frac{2 j}{\omega} q \sqrt{\omega-Q_{+}^{2}}, \quad\left\langle J_{y}\right\rangle=-\frac{2 j}{\omega} p \sqrt{\omega-Q_{+}^{2}} .
$$

Podemos observar que estes valores médios estão relacionados pela transformação canônica $q \rightarrow-p$, $p \rightarrow-q$.

Passemos agora ao cálculo dos valores médios de $J_{x}^{2}$ e $J_{y}^{2}$,

$$
\begin{aligned}
& J_{x}^{2}=+\frac{1}{4}\left(J_{+}^{2}+J_{+} J_{-}+J_{-} J_{+}+J_{-}^{2}\right)=+\frac{1}{4}\left[J_{+}^{2}+J_{-}^{2}+2\left(J^{2}-J_{z}^{2}\right)\right], \\
& J_{y}^{2}=-\frac{1}{4}\left(J_{+}^{2}-J_{+} J_{-}-J_{-} J_{+}+J_{-}^{2}\right)=-\frac{1}{4}\left[J_{+}^{2}+J_{-}^{2}-2\left(J^{2}-J_{z}^{2}\right)\right],
\end{aligned}
$$


onde usamos também o operador de Casimir (2.4). Como os elementos de matriz do operador de Casimir dependem apenas de $j$, o seu valor médio é simplesmente $j(j+1)$. Resta-nos então o cálculo do valor médio de $J_{+}^{2}+J_{-}^{2}$,

$$
\left\langle J_{+}^{2}+J_{-}^{2}\right\rangle=\frac{x^{j}}{(1+x)^{2 j}} \sum_{m^{\prime}=-j}^{j} \sum_{m=-j}^{j} \tau^{* m^{\prime}} \tau^{m}\left(\begin{array}{c}
2 j \\
j+m^{\prime}
\end{array}\right)^{\frac{1}{2}}\left(\begin{array}{c}
2 j \\
j+m
\end{array}\right)^{\frac{1}{2}}\left\langle m^{\prime}\left|J_{+} J_{+}+J_{-} J_{-}\right| m\right\rangle .
$$

Os elementos de matriz $\left\langle m^{\prime}\left|J_{ \pm} J_{ \pm}\right| m\right\rangle$ podem ser escritos explicitamente com o auxílio de (2.5),

$$
\left\langle m^{\prime}\left|J_{ \pm} J_{ \pm}\right| m\right\rangle=\sqrt{\left(j \pm m^{\prime}\right)\left(j \mp m^{\prime}+1\right)} \sqrt{(j \mp m)(j \pm m+1)}\left\langle m^{\prime} \mp 1 \mid m \pm 1\right\rangle,
$$

onde os operadores $J_{ \pm}$atuaram à esquerda e à direita, respectivamente. Substituindo (A.5) em (A.4), teremos

$$
\begin{aligned}
\left\langle J_{+}^{2}+J_{-}^{2}\right\rangle & =\frac{x^{j}}{(1+x)^{2 j}}\{ \\
& \sum_{m^{\prime}=j}^{-j} \sum_{m=j}^{-j} \tau^{* m^{\prime}} \tau^{m}(2 j) !\left[\frac{\left(j+m^{\prime}\right)\left(j-m^{\prime}+1\right)}{\left(j+m^{\prime}\right) !\left(j-m^{\prime}\right) !} \frac{(j-m)(j+m+1)}{(j-m) !(j+m) !}\right]^{\frac{1}{2}}\left\langle m^{\prime}-1 \mid m+1\right\rangle+ \\
& \left.\sum_{m^{\prime}=j}^{-j} \sum_{m=j}^{-j} \tau^{* m^{\prime}} \tau^{m}(2 j) !\left[\frac{\left(j-m^{\prime}\right)\left(j+m^{\prime}+1\right)}{\left(j-m^{\prime}\right) !\left(j+m^{\prime}\right) !} \frac{(j+m)(j-m+1)}{(j+m) !(j-m) !}\right]^{\frac{1}{2}}\left\langle m^{\prime}+1 \mid m-1\right\rangle\right\} \\
& =\frac{x^{j}}{(1+x)^{2 j}}\left\{\begin{array}{c}
2 j \\
-j
\end{array}\right)^{\frac{1}{2}}\left(\begin{array}{c}
2 j \\
j+m+1
\end{array}\right)^{\frac{1}{2}}\left\langle m^{\prime}+1 \mid m-1\right\rangle+ \\
& \sum_{m^{\prime}=j} \sum_{m=j}^{* j} \tau^{* m^{\prime}} \tau^{m}\left(j-m^{\prime}+1\right)(j+m+1)\left(\begin{array}{c}
2 j \\
j+m^{\prime}-1
\end{array}\right) \\
& \left.\sum_{m^{\prime}=j}^{-j} \sum_{m=j}^{-j} \tau^{* m^{\prime}} \tau^{m}\left(j+m^{\prime}+1\right)(j-m+1)\left(\begin{array}{c}
2 j \\
j+m^{\prime}+1
\end{array}\right)\left(\begin{array}{c}
2 j \\
j+m-1
\end{array}\right)^{\frac{1}{2}}\left\langle m^{\prime}-1 \mid m+1\right\rangle\right\} \\
& =\frac{x^{j}}{(1+x)^{2 j}}\left(\tau^{2}+\tau^{* 2}\right) \sum_{m=j-2}^{-j}\left(\begin{array}{c}
2 j \\
j+m+1
\end{array}\right)(j+m+1)(j-m-1) x^{m} \\
& =\frac{x^{-1}}{(1+x)^{2 j}}\left(\tau^{2}+\tau^{* 2}\right) \sum_{m=j-1}^{-j+1}\left(\begin{array}{c}
2 j \\
j+m
\end{array}\right)\left(j^{2}-m^{2}\right) x^{j+m} \cdot
\end{aligned}
$$

Esta última soma pode ser efetuada sem dificuldades,

$$
\left\langle J_{+}^{2}+J_{-}^{2}\right\rangle=2 j(2 j-1) \frac{\tau^{2}+\tau^{* 2}}{(1+x)^{2}}=\frac{4 j(2 j-1)}{\omega^{2}}\left(\omega-Q_{+}^{2}\right) Q_{-}^{2},
$$

onde usamos as variáveis auxiliares $Q_{ \pm}$definidas em (2.55). Portanto, usando também (2.52), os valores médios $\left\langle J_{x}^{2}\right\rangle$ e $\left\langle J_{y}^{2}\right\rangle$ podem ser escritos como

$$
\begin{aligned}
\left\langle J_{x}^{2}\right\rangle & =+\frac{1}{4}\left\{2 j(2 j-1) \frac{\tau^{2}+\tau^{* 2}}{(1+x)^{2}}+2\left[j(j+1)-2 j \frac{x}{(1+x)^{2}}+j^{2}\left(\frac{1-x}{1+x}\right)^{2}\right]\right\} \\
& =\frac{1}{2} j-\frac{2 j(2 j-1)}{4} \frac{\left(\tau+\tau^{*}\right)^{2}}{(1+x)^{2}} \\
\left\langle J_{y}^{2}\right\rangle & =-\frac{1}{4}\left\{2 j(2 j-1) \frac{\tau^{2}+\tau^{* 2}}{(1+x)^{2}}-2\left[j(j+1)-2 j \frac{x}{(1+x)^{2}}+j^{2}\left(\frac{1-x}{1+x}\right)^{2}\right]\right\} \\
& =\frac{1}{2} j-\frac{2 j(2 j-1)}{4} \frac{\left(\tau-\tau^{*}\right)^{2}}{(1+x)^{2}} .
\end{aligned}
$$

Quando re-escritas em termos das variáveis canônicas (2.41), estas expressões tornam-se em

$$
\begin{aligned}
& \left\langle J_{x}^{2}\right\rangle=\frac{j}{2}-\frac{2 j(2 j-1)}{\omega^{2}}\left(q^{4}-\omega q^{2}+q^{2} p^{2}\right), \\
& \left\langle J_{y}^{2}\right\rangle=\frac{j}{2}-\frac{2 j(2 j-1)}{\omega^{2}}\left(p^{4}-\omega p^{2}+q^{2} p^{2}\right) .
\end{aligned}
$$


Podemos observar que estes dois valores médios estão relacionados pela transformação canônica $p \rightarrow q$, $q \rightarrow p$, ou seja, eles possuem os mesmos valores médios, como esperado por argumentos de simetria.

Os valores médios de $J_{x}$ e $J_{y}$ também podem ser calculados de outra forma. Por exemplo, os valores médios de $J_{y}^{k}$ podem ser obtidos diretamente se usarmos a expansão (2.15) no estado coerente (2.36),

$$
\left\langle J_{y}^{k}\right\rangle=\frac{x^{j}}{(1+x)^{2 j}} \sum_{m, \bar{m}} \tau^{m}\left(\tau^{*}\right)^{\bar{m}}\left(\begin{array}{c}
2 j \\
j+m
\end{array}\right)^{\frac{1}{2}}\left(\begin{array}{c}
2 j \\
j+\bar{m}
\end{array}\right)^{\frac{1}{2}} \sum_{m_{y}} m_{y}^{k}\left\langle m_{y} \mid \bar{m}\right\rangle^{*}\left\langle m_{y} \mid m\right\rangle,
$$

onde $J_{z}|j, m\rangle=m|j, m\rangle, J_{y}\left|j, m_{y}\right\rangle=m_{y}\left|j, m_{y}\right\rangle$ e o coeficiente $\left\langle m_{y} \mid m\right\rangle$ é dado em (2.16). Inconvenientemente, estas somas não podem ser realizadas facilmente para um valor literal de $j$, mas podem ser executadas rapidamente para qualquer valor numérico de $j$, usando computação algébrica. Desta forma, após $k-1$ valores explícitos de $\left\langle J_{y}^{k}\right\rangle$ terem sido calculados, uma interpolação polinomial em $j$ pode ser efetuada para fornecer uma expressão geral em $j$ para $\left\langle J_{y}^{k}\right\rangle$, para cada valor de $k$. Assim, os sete primeiros valores médios $\left\langle J_{y}^{k}\right\rangle$ estão listados abaixo:

$$
\begin{aligned}
&\left\langle J_{y}^{0}\right\rangle= 1, \\
&\left\langle J_{y}^{1}\right\rangle= \frac{\delta}{\chi} j, \\
&\left\langle J_{y}^{2}\right\rangle=\left(\frac{\delta}{\chi}\right)^{2} j^{2}+\frac{1}{2}\left(\frac{\kappa}{\chi}\right)^{2} j, \\
&\left\langle J_{y}^{3}\right\rangle=\left(\frac{\delta}{\chi}\right)^{3} j^{3}+\frac{3}{2} \frac{\delta \kappa^{2}}{\chi^{3}} j^{2}-\frac{1}{2} \frac{\delta \kappa^{2}}{\chi^{3}} j, \\
&\left\langle J_{y}^{4}\right\rangle=\left(\frac{\delta}{\chi}\right)^{4} j^{4}+3 \frac{\delta^{2} \kappa^{2}}{\chi^{4}} j^{3}+\frac{1}{4} \frac{\kappa^{2}\left(3 \kappa^{2}-8 \delta^{2}\right)}{\chi^{4}} j^{2}-\frac{1}{4} \frac{\kappa^{2}\left(\kappa^{2}-2 \delta^{2}\right)}{\chi^{4}} j, \\
&\left\langle J_{y}^{5}\right\rangle=\left(\frac{\delta}{\chi}\right)^{5} j^{5}+5 \frac{\delta^{3} \kappa^{2}}{\chi^{5}} j^{4}+\frac{5}{4} \frac{\delta \kappa^{2}\left(3 \kappa^{2}-4 \delta^{2}\right)}{\chi^{5}} j^{3}-\frac{5}{4} \frac{\delta \kappa^{2}\left(3 \kappa^{2}-2 \delta^{2}\right)}{\chi^{5}} j^{2}+\frac{1}{2} \frac{\delta \kappa^{2}\left(2 \kappa^{2}-\delta^{2}\right)}{\chi^{5}} j, \\
&\left\langle J_{y}^{6}\right\rangle=\left(\frac{\delta}{\chi}\right)^{6} j^{6}+\frac{15}{2} \frac{\delta^{4} \kappa^{2}}{\chi^{6}} j^{5}+\frac{5}{4} \frac{\delta^{2} \kappa^{2}\left(9 \kappa^{2}-6 \delta^{2}\right)}{\chi^{6}} j^{4}+\frac{15}{8} \frac{\kappa^{2}\left[\chi^{4}-3 \delta^{2}\left(4 \kappa^{2}-\delta^{2}\right)\right]}{\chi^{6}} j^{3} \\
&-\frac{1}{8} \frac{\kappa^{2}\left[15 \chi^{4}-\delta^{2}\left(128 \kappa^{2}-9 \delta^{2}\right)\right]}{\chi^{6}} j^{2}+\frac{1}{4} \frac{\kappa^{2}\left(2 \chi^{4}-15 \delta^{2} \kappa^{2}\right)}{\chi^{6}} j,
\end{aligned}
$$

com

$$
\delta=i\left(\tau-\tau^{*}\right)=\frac{2}{\sqrt{\omega}} q, \quad \chi^{2}=1+|\tau|^{2}=\frac{\omega}{\omega-Q_{+}^{2}}, \quad \kappa^{2}=\chi^{2}-\delta^{2},
$$

onde aplicamos a transformação canônica $\{q \rightarrow p, p \rightarrow q\}$ em (2.41),

$$
\tau=\frac{z}{\sqrt{1-|z|^{2}}}, \quad z=\frac{1}{\sqrt{\omega}}(p+i q), \quad Q_{+}^{2}=q^{2}+p^{2}, \quad \omega=4 j .
$$




\section{Apêndice B}

\section{Realização de Morse para a álgebra}

\section{$\mathrm{su}(2)$}

O propósito deste apêndice é mostrar que a realização (3.29) para os geradores da álgebra su(2) admite as funções de Morse (3.24), multiplicadas pela fase $\mathrm{e}^{-2 i m_{y} \phi} \mathrm{em}$ (3.23), como base para suas representações irredutíveis, $\Psi_{j m_{y}}(\phi, z)=\left\langle\phi, z \mid j, m_{y}\right\rangle$. Para isto, temos que mostrar que a ação dos geradores $J_{ \pm}$nas funções $\Psi_{j m_{y}}(\phi, z)$ reproduzem os resultados (2.5). Iniciemos pela ação de $J_{+}$,

$$
J_{+} \Psi_{j m_{y}}(\phi, z)=A_{j m_{y}} \mathrm{e}^{2 i\left(m_{y}+1\right) \phi} \mathrm{e}^{-\frac{z}{2}} z^{\left(m_{y}+1\right)}\left(\frac{d}{d z}-\frac{d^{2}}{d z^{2}}\right) L_{j-m_{y}}^{2 m_{y}} .
$$

Fazendo uso das relações

$$
\frac{d L_{n}^{\alpha}}{d z}=-L_{n-1}^{\alpha+1}, \quad L_{n}^{\alpha}=L_{n}^{\alpha+1}-L_{n-1}^{\alpha+1}
$$

para os polinômios de Laguerre, as derivadas em (B.1) podem ser eliminadas,

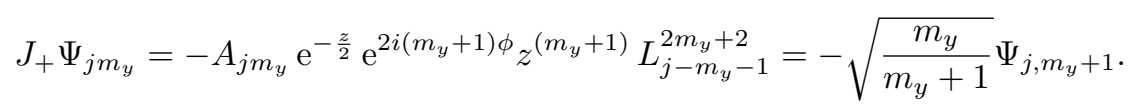

A mesma verificação para o gerador $J_{-}$é um pouco mais extensa, pois

$$
J_{-} \Psi_{j m_{y}}=A_{j m_{y}} \mathrm{e}^{-\frac{z}{2}} e^{2 i\left(m_{y}-1\right) \phi} z^{\left(m_{y}-1\right)} T
$$

onde

$$
T=\left[2 m_{y}\left(1-2 m_{y}+z\right)\right] L_{j-m_{y}}^{2 m_{y}}+\left(z-4 m_{y}\right) z\left(\frac{d}{d z}-z^{2} \frac{d^{2}}{d z^{2}}\right) L_{j-m_{y}}^{2 m_{y}} .
$$

Neste caso, devemos usar primeiro a seguinte relação de recorrência:

$$
z^{2} \frac{d^{2}}{d z^{2}} L_{n}^{\alpha}=(z-\alpha-1) z \frac{d}{d z} L_{n}^{\alpha}-n z L_{n}^{\alpha}
$$

Assim, a expressão (B.5) pode ser re-escrita na forma

$$
T=\left[4 m^{2}-2 m-(j+m) z\right] L_{j-m_{y}}^{2 m_{y}}+(2 m-1) \frac{d}{d z} L_{j-m_{y}}^{2 m_{y}} .
$$

Esta equação pode ser simplificada um pouco mais através da relação de recorrência

$$
z \frac{d}{d z} L_{n}^{\alpha}=n L_{n}^{\alpha}-(n+\alpha) L_{n-1}^{\alpha}
$$

Substituindo esta relação em (B.7), obteremos

$$
T=\left[2 m^{2}-m(N+1)-j\right] L_{j-m_{y}}^{2 m_{y}}-(j+m) z L_{j-m_{y}}^{2 m_{y}}-\left[2 m^{2}+(2 j-1) m-j\right] L_{j-m_{y}-1}^{2 m_{y}} .
$$


O termo $z L_{j-m_{y}}^{2 m_{y}}$ pode ser eliminado pela relação de recorrência

$$
x L_{n}^{\alpha}=(2 n+\alpha+1) L_{n}^{\alpha}-(n+1) L_{n+1}^{\alpha}-(n+\alpha) L_{n-1}^{\alpha} .
$$

Assim, após substituirmos (B.10) em (B.9), obteremos

$$
T=\left(m^{2}-m-j^{2}-j\right)\left(2 L_{j-m_{y}}^{2 m_{y}}-L_{j-m_{y}-1}^{2 m_{y}}-L_{j-m_{y}+1}^{2 m_{y}}\right) .
$$

Como precisamos deixar todos os termos nesta expressão na forma $L_{j-m_{y}+1}^{2\left(m_{y}-1\right)}$, então precisaremos de mais duas relações,

$$
\begin{aligned}
L_{j-m_{y}}^{2 m_{y}} & =L_{j-m_{y}}^{2 m_{y}-1}+L_{j-m_{y}-1}^{2 m_{y}}, \\
L_{j-m_{y}+1}^{2 m_{y}} & =L_{j-m_{y}+1}^{2 m_{y}-2}-2 L_{j-m_{y}}^{2 m_{y}-1}+L_{j-m_{y}-1}^{2 m_{y}} .
\end{aligned}
$$

Finalmente, substituindo estas relações em (B.11), obteremos

$$
T=-(j-m+1)(j+m) L_{j-m_{y}+1}^{2 m_{y}-2} .
$$

Portanto, o resultado final da ação de $J_{-}$é

$$
J_{-} \Psi_{j m_{y}}=-A_{j m_{y}}(j+m)(j-m+1) \mathrm{e}^{-\frac{z}{2}} \mathrm{e}^{2 i\left(m_{y}-1\right) \phi} z^{\left(m_{y}-1\right)} L_{j-m_{y}+1}^{2 m_{y}-2}=-\sqrt{\frac{m_{y}}{m_{y}-1}} \Psi_{j, m_{y}-1} .
$$

Os resultados (B.3) e (B.15) sugerem que as novas funções

$$
\bar{\Psi}_{j m_{y}}=\frac{(-1)^{m_{y}}}{\sqrt{m_{y}}} \Psi_{j m_{y}}
$$

comportam-se como auto-funções de $J_{y}$. 


\section{Bibliografia}

[1] G. Herzberg. Molecular Spectra and Molecular Structure. I. Spectra of Diatomic Molecules. Van Nostrand Reinhold, 1950. 1, 2, 19

[2] G. Herzberg. Molecular Spectra and Molecular Structure II. Infrared and Raman Spectra of Polyatomic Molecules. D. Van Nostrand, 1964. 1, 19

[3] I. N. Levine. Quantum Chemistry. Prentice-Hall, 1991. 1

[4] P. W. Atkins and R. S. Friedman. Molecular Quantum Mechanics. Oxford, 2001. 1

[5] Luiz A. B. San Martin. Álgebras de Lie. Unicamp, 1999. 1

[6] B. G. Wybourne. Classical Group for Physicists. John Wiley, 1974. 1, 2

[7] R. Gilmore. Lie Groups, Lie Algebras and Some of Their Applications. Wiley, 1974. 1, 2, 9

[8] A. O. Barut and R. Raczka. Theory of Group Representations and Applications. World Scientific, 1986. 1, 2, 9

[9] Jialun Ping, Fan Wang, and Jin-Quan Chen. Group Representation Theory for Physicists. World Scientific, 2nd edition, 2002. 1, 2

[10] M. S. Lie. Sophus Lie's 1880 transformation group paper. In N. Wallach and R. Hermann, editors, Lie Groups: History, Frontiers and Applications, volume I. Math Sci Press, 1975. 1

[11] E. P. Wigner. Group Theory and Its Application to the Quantum Mechanics of Atomic Spectra, volume 5 of Pure and Applied Physics. Academic Press, 1959. 1

[12] H. Weyl. Theory of Groups and Quantum Mechanics. Dover, 1950. 1

[13] H. Weyl. Symmetry. Princeton, 1952. 1

[14] S. D. Drell. The Richtmyer memorial lecture - when is a particle? Am. J. Phys., 46(6):597, 1978.1

[15] A. Salam. Gauge unification of fundamental forces. Rev. Mod. Phys., 52(3):525, 1980. 1

[16] M. Gell-man and Y. Ne'eman. The Eightfold Way. Benjamim, 1964. 1

[17] J. L. Lopes. Gauge Field Theories: An Introduction. Oxford, 1981. 1

[18] C. Quig. Gauge Theories of Strong, Weak, and Electromagnetic Interactions, volume 56 of Frontiers in Physics. Benjamin-Cummings, 1983. 1

[19] T.-P. Cheng and L.-F. Li. Gauge Theory of Elementary Particle Physics. Oxford, 1984.1

[20] A. Bohm and R. B. Teese. Spectrum generating group of the symmetric top molecule. J. Math. Phys., 17(1):94, 1976. 1

[21] A. Bohm and Y. Ne'eman. Dynamical groups and spectrum generating algebras. In A. Bohm, Y. Ne'eman, and A. O. Barut, editors, Dynamical Groups and Spectrum Generating Algebras, volume 1, page 3. World Scientific, 1988. 1 
[22] F. Iachello and A. Arima. The Interacting Boson Model. Cambridge, 1987. 1

[23] D. Bonatsos. Interacting Boson Models of Nuclear Structure. Clarendon, 1988. 1

[24] A. Frank and P. van Isacker. Algebraic Methods in Molecular and Nuclear Structure Physics. John Wiley, 1994. 1, 19, 29

[25] F. Iachello and R. D. Levine. Algebraic Theory of Molecules. Oxford, 1995. 1, 11, 19, 29

[26] M. E. Kellman. Algebraic methods in spectroscopy. Annu. Rev. Phys. Chem., 46:395, 1995. 1, 2

[27] Y. Alhassid, F. Gürsey, and F. Iachello. Group theory approach to scattering. Ann. Phys., 148:346, 1983. 1, 13, 15, 16, 17, 18, 19

[28] I. L. Cooper. An integrated approach to ladder and shift operators for the Morse oscillator, radial Coulomb and radial oscillator potencials. J. Phys. A: Math. Gen., 26:1601, 1993. 1, 13, 19

[29] Shi-Hai Dong, R. Lemus, and A. Frank. Ladder operators for the Morse potential. Int. J. Quant. Chem., 86:433, 2002. 1, 15

[30] T. Müller P. H. Vaccaro, F. Pérez-Bernal, and F. Iachello. Algebraic approach for the calculation of polyatomic Franck-Condon factors: application to the vibronically resolved absortion spectrum of disulfur monoxide $\mathrm{S}_{2} \mathrm{O}$. Chem. Phys. Letts., 329:271, 2000. 2, 19, 55

[31] M. E. Kellman. Dynamical analysis of highly excited vibrational spectra: Progress and application. In Hai-Lung Dai and R. W. Field, editors, Advanced Series in Physical Chemistry, volume 4, page 943. World Scientific, 1995. 2

[32] M. Golubitsky, I. Stewart, and D. G. Schaeffer. Singularities and Groups in Bifurcation Theory. Volume II, volume 69 of Applied Mathematical Sciences. Springer-Verlag, 1988. 2

[33] R. Gilmore. Catastrophe Theory for Scientists and Engineers. Dover, 1981. 2, 11

[34] B. I. Zhilinskii. Symmetry, invariants, and topology in molecular models. Phys. Reports, 341(1-6):85, 2001. 2

[35] T. Müller, P. Dupré, P. H. Vaccaro, F. Pérez-Bernal, M. Ibrahim, and F. Iachello. Algebraic approach for the calculation of polyatomic Franck-Condon factors. Application to the vibronically resolved emission spectrum of $\mathrm{S}_{2}$ O. Chem. Phys. Letts., 292:243, 1998. 2

[36] F. Iachello, F. Perez-Bernal, and P. H. Vaccaro. A novel algebraic scheme for describing nonrigid molecules. Chem. Phys. Lett., 375(3-4):309, 2003. 2

[37] W. C. Stwalley, W. T. Zemke, and S. C. Yang. Spectroscopy and structure of the alkali hydride diatomic molecules and their ions. J. Phys. Chem. Ref. Data, 20(1):153, 1991. 2, 29

[38] W. C. Stwalley and W. T. Zemke. Spectroscopy and structure of the lithium hydride diatomic molecules and ions. J. Phys. Chem. Ref. Data, 22(1):87, 1993. 2, 29, 30, 32, 34, 35, 37, 38, 47

[39] K. K. Verma, M. E. Koch, and W. C. Stwalley. Observation of levels near dissociation in the $X^{1} \Sigma_{g}^{+}$ state of ${ }^{7} \mathrm{Li}_{2}$. J. Chem. Phys., 78(6):3614, 1983. 2

[40] B. Barakat, R. Bacis, F. Carrot, S. Churassy, P. Crozet, and F. Martin. Extensive analysis of the $X^{1} \Sigma_{g}^{+}$ground state of ${ }^{7} \mathrm{Li}_{2}$ by laser-induced fluorescence Fourier transform spectrometry. Chem. Phys., 102(1-2):215, 1986. 2

[41] C. Linton, F. Martin, I. Russier, A. J. Ross, P. Crozet, S. Churassy, and R. Bacis. Observation and analysis of the $A^{1} \Sigma_{u}^{+}$state of ${ }^{6} \mathrm{Li}_{2}$ from $\nu=0$ to the dissociation limit. J. Mol. Spectrosc., 175:340, 1996. 2

[42] K. Urbanski, S. Antonova, A. Yiannopoulou, A. M. Lyyra, Li Li, and W. C. Stwalley. All optical triple resonance spectroscopy of the $A^{1} \Sigma_{u}^{+}$state of ${ }^{7} \mathrm{Li}_{2}$. J. Chem. Phys., 104(8):2813, 1996. 2

[43] I. L. Cooper. Diatomic potential functions derived from accurate fits to vibrational energy levels using algebraic theory. J. Phys. Chem. A, 102:9565, 1998. 2, 11, 19, 20 
[44] J. Tennyson, N.F. Zobov, R. Williamson, O.L. Polyansky, and P.F. Bernath. Experimental energy levels of the water molecule. J. Phys. Chem. Ref. Data, 30(735), 2001. 3

[45] K. Yamanouchi, S. Takeuchi, and S. Tsuchiya. Vibrational level structure of higly excited $\mathrm{SO}_{2}$ in the electronic ground state. II. Vibrational assignment by dispersed fluorescence and stimulated emission pumping spectroscopy. J. Chem. Phys., 92(2):4044, 1990. 3

[46] A. Delon and R. Jost. Laser induced dispersed fluorescence spectra of jet cooled $\mathrm{NO}_{2}$ : The complete set of vibracional levels up to $10000 \mathrm{~cm}^{-1}$ and the onset of the $\tilde{X}^{2} A_{1}-\tilde{A}^{2} B_{2}$. J. Chem. Phys., $95(8): 5686,1991.3$

[47] R. J. Bouwens, J. A. Hammerschimidt, M. M. Grzeskowiak, T. A. Stegink, P. M. Yorba, and W. F. Polik. Pure vibrational spectroscopy of $\mathrm{S}_{0}$ formaldehyde by dispersed fluorescence. J. Chem. Phys., 104(2):460, 1996. 3

[48] A. Messiah. Quantum Mechanics. John Wiley, 1976. 5, 6, 18

[49] E. Merzbacher. Quantum Mechanics. John Wiley, 1998. 5, 6

[50] Wu-Ki Tung. Group Theory in Physics. World Scientific, 1985. 6

[51] J. Schwinger. On angular momentum. In L. C. Biedenharn and H. van Dam, editors, Quantum Theory of Angular Momentum. Academic Press, 1965. 6

[52] M. Jimbo, editor. Yang-Baxter Equation in Integrable Systems, volume 10 of Advanced Series in Mathematical Physics. World Scientific, 1980. 7, 8

[53] L. C. Biedenharn and M. A. Lohe. Quantum Groups Symmetry and q-Tensor Algebras. World Scientific, 1995. 7, 8

[54] Vyjayanthi Chari and Andrew Pressley. A Guide To Quantum Groups. Cambridge University, 1994. 7

[55] M. Jimbo. A q-difference analogue of $U(g)$ and the Yang-Baxter equation. Lett. Math. Phys., 10:63, 1985. 7

[56] Z. Chang. Quantum group and quantum symmetry. Phys. Report., 262:137, 1995. 7

[57] F. C. Alcaraz, S. R. Salinas, and W. F. Wreszinski. Anisotropic ferromagnetic quantum domains. Phys. Rev. Lett., 75(5):930, 1995. 7

[58] F. C. Alcaraz and Yu. G. Stroganov. The finite size SU(3) Perk-Schultz model with deformation parameter $q=\exp (2 i \pi / 3)$. J. Phys. A: Math. Gen., 35:3805, 2002. 7

[59] D. Bonatsos and C. Daskaloyannis. Deformed oscillator for the Morse potential. Chem. Phys. Letters, 203(2-3):150, 1993. 7

[60] A. Kundu and Y. J. Ng. q-deformed angular momentum operator and possible application to triatomic molecules. Phys. Lett. A, 197:221, 1995. 7

[61] I. L. Cooper and R. K. Gupta. q-deformed Morse oscillator. Phys. Rev. A, 52(2):941, 1995.7

[62] Jin-Quan Chen. Group Representation Theory for Physicists. World Scientific, 1989. 7

[63] M. Irac-Astaud and C. Quesne. Unitary representations of the quantum algebra $\mathrm{su}_{q}(2)$ on a real two-dimensional sphere for $q \in R^{+}$or generic $q \in S^{1}$. J. Math. Phys., 40(6):3146, 1999. 8, 53

[64] M. Novaes, J. E. M. Hornos, and E. S. Bernardes. Harmonic functions of $\operatorname{su}_{q}(2)$ for $q \in \mathbb{R}$ and $q \rightarrow S^{1}$. J. Phys. A: Math. Gen., 36(24):6733, 2003. 8, 53

[65] J. R. Klauder and B. S. Skagerstam. Coherent States, Applications in Physics and Mathematical Physics. World Scientific, 1985. 8

[66] A. M. Perelomov. Generalized Coherent States and Their Applications. Springer, 1986. 8 
[67] Wei-Min Zhang, Da Hsuan Feng, and R. Gilmore. Coherent states: theory and some applications. Rev. Mod. Phys., 62(4):867, 1990. 8, 9, 10

[68] M. Novaes. Mecânica Quântica no espaço de fase: II. estados coerentes. Rev. Bras. Ens. Fis., $24(4): 437,2002.8$

[69] M. A. Marchiolli. Mecânica Quântica no espaço de fase: I. formulação de Weyl-Wigner. Rev. Bras. Ens. Fis., 24(4):421, 2002. 8

[70] W. M. Zhang, C. C. Martens, D. H. Feng, and J. M. Yuan. Dynamical symmetry breaking and quantum nonintegrability. Phys. Rev. Lett., 61(19):2167, 1988. 10

[71] C Quesne. New q-deformed coherent states with an explicitly known resolution oj unity. J. Phys. A: Math. Gen., 35(43):9213, 2002. 10, 53

[72] R. D. Levine and C. E. Wulfman. Energy transfer to a Morse oscillator. Chem. Phys. Letters, $60(3): 372,1979.11,19$

[73] O. S. van Roosmalen, R. D. Levine, and A. E. L. Dieperink. The geometrical-classical limit of algebraic hamiltonians for molecular vib-rotational spectra. Chem. Phys. Letts., 101(6):512, 1983. 11,19

[74] S. K. Kim, I. L. Cooper, and R. D. Levine. The algebraic hamiltonian for diatomic molecules in the vibron model. Chem. Phys., 106:1, 1986. 11

[75] I. L. Cooper and R. K. Gupta. Application of $U(2) \supset O(2)$ and $U(2) \supset U(1)$ dynamical symmetries to diatomic molecular vibrations. Phys. Rev. A, 55(6):4112, 1997. 11, 54

[76] P. M. Morse. Diatomic molecules according to the wave mechanics II: Vibrational levels. Phys. Rev., 34:57, 1929. 13

[77] M. Berrondo and A. Palma. The algebraic approach to the Morse oscillator. J. Phys. A: Math. Gen., 13:773, 1980. 13, 17

[78] L. J. Slater. Confluent hypergeometric functions. In M. Abramowitz and I. A. Stegun, editors, Handbook of Mathematical Functions, page 503. Dover, 1964. 14

[79] A. Matsumoto. Generalised matrix elements in discrete and continuum states for the Morse potential. J. Phys. B:At. Mol. Opt. Phys., 21:2863, 1988. 14, 16, 27

[80] G. Pöschl and S. Teller. Bemerkungen zur quantenmechanik des anharmonischen oszillators. $Z$. Physik, 83(143), 1933. 17

[81] A. Erdélyi. Higher transcendental functions. In Harry Bateman, editor, The Bateman Manuscript Project. McGraw-Hill, 1953. 18

[82] S. Flügge. Pratical Quantum Mechanics. Springer-Verlarg, 1974. 18

[83] G. B. Arfken and H. J. Weber. Mathematical Methods for Physicists. Academic, 1995. 18

[84] A. Frank, R. Lemus, R. Bijker, F. Pérez-Bernal, and J. M. Arias. A general algebraic model for molecular vibrational spectroscopy. Ann. Phys., 252:211, 1996. 19

[85] S. Oss. Algebraic models in molecular spectroscopy. In I. Prigogine and S. A. Rice, editors, Advances in Chemical Physics, volume XCIII, page 455. Wiley, 1996. 19

[86] Jye-Jong Chen, Wei-Tzou Luh, and Gwang-Hi Jeung. Spectroscopic study of the $C^{1} \Sigma^{+}$state of ${ }^{7}$ LiH. J. Chem. Phys., 110(9):4402, 1999. 29

[87] Jye-Jong Chen, Wei-Tzou Luh, Gwang-Hi Jeung, and F. X. Gadéa. Thee $D^{1} \Sigma^{+}$state of ${ }^{7}$ LiH. J. Chem. Phys., 113(2):683, 2000. 29

[88] F. Gemperle and F. X. Gadéa. Breakdown of the Born-Oppenheimer approach for a diatomic molecule: LiH in the D state. Europhy. Letts., 48(5):513, 1999. 29 
[89] W. T. Zemke and W. C. Stwalley. Radiative transition probalities for the $A^{1} \Sigma^{+}-X^{1} \Sigma^{+}$bands of ${ }^{7}$ LiH. J. Chem. Phys., 68(10):4619, 1978. 29, 33

[90] W. T. Zemke and W. C. Stwalley. Radiative transition probalities, lifetimes, and dipole moments for all vibrational levels in the $A^{1} \Sigma^{+}$state of ${ }^{7} \mathrm{LiH}$. J. Chem. Phys., 73(11):5584, 1980. 29

[91] A. L. G. Rees. The calculation of potential-energy curves from band-spectroscopic data. Proc. Phys. Soc. Lond., 59:998, 1947. 31

[92] W. C Stwalley. Atom-atom interactions from spectroscopy. In D. W. Smith and D. B. McRae, editors, Energy, Structure and Reactivity, page 259. Wiley, 1973. 31

[93] W. M. Kosman and J. Hinze. Inverse perturbation analysis - Improving accuracy of potential-energy curves. J. Mol. Spectrosc., 56(1):93, 1975. 31

[94] C. R. Vidal and H. Scheingraber. Determination of diatomic molecular-constants using an inverted perturbation approach - Application to $A^{1} \Sigma_{u}^{+}-X^{1} \Sigma_{u}^{+}$system of $\mathrm{Mg}_{2}$. J. Mol. Spectrosc., 65(1):46, 1977. 31

[95] F. Iachello and S. Oss. Algebraic approach to molecular spectra: two-dimensional problems. $J$. Chem. Phys., 104(18):6956, 1996. 55

[96] F. Iachello, S. Oss, and L. Viola. Vibrational analysis of monofluoroacetylene (HCCF) in the vibron model. Mol. Phys., 78(3):545, 1993. 55

[97] E. S. Bernardes, Y. M. M. Hornos, and J. E. M. Hornos. Dynamical symmetry in the vibrational overtone spectrum of monofluoroacetylene (HCCF). Chem. Phys., 213:17, 1996. 55

[98] E. S. Bernardes and J. E. M. Hornos. The overtone spectrum of monochloroacetylene ( $\mathrm{HCCCl})$ in the algebraic approach. Chem. Phys., 242(3):295, 1999. 55 